\title{
Low-Frequency Gravitational Wave Searches Using Spacecraft Doppler Tracking
}

\author{
J. W. Armstrong \\ Jet Propulsion Laboratory \\ California Institute of Technology \\ Mail Stop 238-725, 4800 Oak Grove Dr. \\ Pasadena, CA 91109-8001, U.S.A. \\ email: John.W.Armstrong@jpl.nasa.gov
}

Accepted: 2 November 2005

Published: 24 January 2006

Revised: 11 January 2016

\begin{abstract}
This paper discusses spacecraft Doppler tracking, the current-generation detector technology used in the low-frequency ( $\sim$ millihertz) gravitational wave band. In the Doppler method the earth and a distant spacecraft act as free test masses with a ground-based precision Doppler tracking system continuously monitoring the earth-spacecraft relative dimensionless velocity $2 \Delta v / c=\Delta \nu / \nu_{0}$, where $\Delta \nu$ is the Doppler shift and $\nu_{0}$ is the radio link carrier frequency. A gravitational wave having strain amplitude $h$ incident on the earth-spacecraft system causes perturbations of order $h$ in the time series of $\Delta \nu / \nu_{0}$. Unlike other detectors, the $\sim 1-10 \mathrm{AU}$ earth-spacecraft separation makes the detector large compared with millihertz-band gravitational wavelengths, and thus times-of-flight of signals and radio waves through the apparatus are important. A burst signal, for example, is time-resolved into a characteristic signature: three discrete events in the Doppler time series. I discuss here the principles of operation of this detector (emphasizing transfer functions of gravitational wave signals and the principal noises to the Doppler time series), some data analysis techniques, experiments to date, and illustrations of sensitivity and current detector performance. I conclude with a discussion of how gravitational wave sensitivity can be improved in the low-frequency band.
\end{abstract}

Keywords: Cassini, Doppler stability, Doppler tracking, frequency stability, gravitational wave detectors, radio

(C) The Author(s). This article is distributed under a Creative Commons Attribution 4.0 International License. http://creativecommons.org/licenses/by/4.0/ 


\section{Imprint / Terms of Use}

Living Reviews in Relativity is a peer-reviewed open access journal published by the Springer International Publishing AG, Gewerbestrasse 11, 6330 Cham, Switzerland. ISSN 1433-8351.

This article is distributed under the terms of the Creative Commons Attribution 4.0 International License (http://creativecommons.org/licenses/by/4.0/), which permits unrestricted use, distribution, and reproduction in any medium, provided you give appropriate credit to the original author(s) and the source, provide a link to the Creative Commons license, and indicate if changes were made. Figures that have been previously published elsewhere may not be reproduced without consent of the original copyright holders.

J. W. Armstrong,

"Low-Frequency Gravitational Wave Searches Using Spacecraft Doppler Tracking", Living Rev. Relativity, 9, (2006), 1.

DOI 10.12942/lrr-2006-1. 


\section{Article Revisions}

Living Reviews supports two ways of keeping its articles up-to-date:

Fast-track revision. A fast-track revision provides the author with the opportunity to add short notices of current research results, trends and developments, or important publications to the article. A fast-track revision is refereed by the responsible subject editor. If an article has undergone a fast-track revision, a summary of changes will be listed here.

Major update. A major update will include substantial changes and additions and is subject to full external refereeing. It is published with a new publication number.

For detailed documentation of an article's evolution, please refer to the history document of the article's online version at http://dx.doi.org/10.12942/lrr-2006-1.

15 Jan 2008: Included three way in Table 1; modified Figure 9 (parallel structure with Figure 12) and corrected typographical error regarding correct factor to isolate dispersive plasma in Section 4.2; updated the propagation noise Figure 10; corrected entry in Table 2 for stochastic spacecraft motion; added short discussion of flyable clocks and discussion of $\mathrm{E}(\mathrm{t})$, two Figures 25 and 26 about the antenna mechanical test, and five references; changed title of Section 8 to include "LISA", updated throughout estimate of launch date.

Page 9: Added three way.

Page 19: Modified figure to discuss the blowup of the acf.

Page 17: Corrected typographical error X-(749/3344) Ka1 to X-(880/3344) Ka1.

Page 20: Updated Figure 10.

Page 28: Corrected entry for stochastic spacecraft motion from $\simeq 3 \times 10^{-16}$ to $\simeq 2 \times 10^{-16}$.

Page 44: Added short discussion of flyable clocks and discussion of $\mathrm{E}(\mathrm{t})$ below. New references Armstrong 1997, Armstrong et al. 2006, Armstrong et al. 2008, Cliche and Shillue 2006, Snel et al. 2007.

Page 46: Added Figure 25.

Page 47: Added Figure 26.

Page 47: Changed section title to include LISA.

11 Jan 2016: New Subsections 4.9, 6.2, and 11 new references were added, 2 were removed.

Page 8: Removed last paragraph on organization of this review.

Page 12: Corrected sign error for $\mu$ to galactic center in Figure 2.

Page 13: Replaced Figure 3 with a different photograph.

Page 17: Added reference Richie-Halford et al. (2009) in new footnote.

Page 20: Replaced Figure 10 with updated version containing new data.

Page 25: New Subsection 4.9 on numerical noise and references to Moyer (1971), Zannoni \& Tortora (2013) and Iess et al. (2014).

Page 37: Added Juno (Bolton et al., 2010; Matousek, 2007) and BepiColombo (Iess and Boscagli, 2001; Benkhoff et al., 2010) to Table 3. 
Page 38: New Subsection 6.2 and Figure 19 on future, e.g., Juno and BepiColombo, observations.

Page 44: Added references to Hinkley et al. (2013) and Tinto et al. (2009); removed Takamoto et al. (2005).

Page 47: Updated discussion of LISA (eLISA, gLISA, and LISA Pathfinder launch date); added Tinto et al. (2014) reference. Removed last paragraph on further possible implications for LISA, thus needed to omit the Synthetic LISA reference (Vallisneri, 2005). 


\section{Contents}

1 Introduction $\quad 7$

2 Notation, Acronyms, and Conventions $r$

3 Gravitational Wave Signal Response 10

4 Apparatus and Principal Noise Sources $r$

4.1 Frequency standard noise . . . . . . . . . . . . . . . . . . . . 15

4.2 Plasma scintillation noise . . . . . . . . . . . . . . . . . . . . 16

4.3 Tropospheric scintillation noise . . . . . . . . . . . . . . . . 18

4.4 Antenna mechanical noise . . . . . . . . . . . . . . . . . . . . . . 22

4.5 Ground electronics noise . . . . . . . . . . . . . . . . . . . . . 22

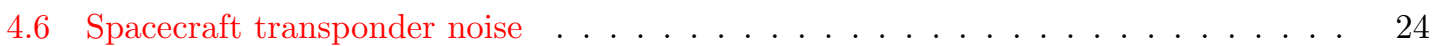

4.7 Thermal noise in the ground and spacecraft receivers . . . . . . . . . . . . . . . 24

4.8 Spacecraft unmodeled motion . . . . . . . . . . . . . . . . . . . . 24

4.9 Numerical noise in orbit removal . . . . . . . . . . . . . . . . . . . . 25

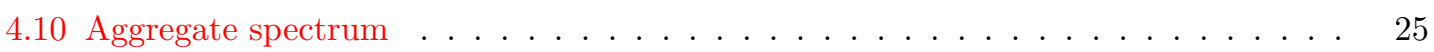

4.11 Summary of noise levels and transfer functions . . . . . . . . . . . . . . 26

5 Signal Processing $\quad \mathbf{2 9}$

5.1 Noise spectrum estimation . . . . . . . . . . . . . . . . . . . . . . . 29

5.2 Sinusoidal and quasiperiodic waves . . . . . . . . . . . . . . . . . . . . . . . . . . . . . . . . . . . . . . .

5.3 Bursts . . . . . . . . . . . . . . . . . . . . . . 30

5.4 Stochastic background . . . . . . . . . . . . . . . . . . 30

5.5 Classification of data intervals based on transfer functions . . . . . . . . . . . . . 31

5.6 Frequency-time representations . . . . . . . . . . . . . . . . . . 31

5.7 Qualifying/disqualifying candidates . . . . . . . . . . . . . . . . . . . . . . . . . . . . . . . . . .

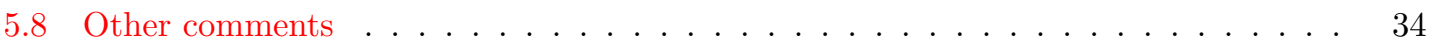

6 Detector Performance $\quad 37$

6.1 Observations to date . . . . . . . . . . . . . . . . . . . 37

6.2 Near-future observations . . . . . . . . . . . . . . . . . . . . . . . . . . 38

6.3 Sensitivity to periodic and quasi-periodic waves . . . . . . . . . . . . . . . . . . . . . . . . . . . . . . . .

6.3.1 Sinusoidal waves and chirps . . . . . . . . . . . . . . . . . . . . . . . . . . . . .

6.3.2 Nonsinusoidal periodic waves . . . . . . . . . . . . . . . . . 40

6.4 Burst waves . . . . . . . . . . . . . . . . . . . . . . 41

6.5 Sensitivity to a stochastic background . . . . . . . . . . . . . . . . . 42

7 Improving Doppler Tracking Sensitivity $\quad 44$

8 The LISA Low-Frequency Detector $\quad 47$

9 Concluding Comments $\quad 49$

$\begin{array}{lr}\text { References } & 51\end{array}$ 


\section{List of Tables}

1 Some acronyms and notation used in this paper. . . . . . . . . . . . . . 9

2 Noise sources and contributions in Cassini-era precision Doppler tracking GW observations. . . . . . . . . . . . . . . . . . . . . 28

3 Spacecraft Doppler gravitational-wave observations. . . . . . . . . . . . . . 37

4 Required improvement in subsystems to improve overall Doppler sensitivity by a factor of 10 relative to Cassini-era performance. . . . . . . . . . . . . . . 45 


\section{Introduction}

Radio communications systems on deep space probes are used for both command and control of the spacecraft (via transmissions from the earth to the spacecraft, the uplink) and for returning telemetry to the ground (via transmission from the spacecraft to the earth, the downlink). These communications systems typically serve two additional purposes: navigation (use of radio ranging and earth-spacecraft Doppler to determine the position and velocity of the probe) and radio science (use of measured radiowave properties - amplitude, frequency, polarization, etc. - explicitly for mission science). Radio science can address several scientific topics including estimation of planetary masses and mass distributions, measurements of planetary ionospheres/atmospheres/rings, studies of planetary shapes and surfaces, observations of the solar wind, and tests of relativistic gravity.

This article describes a radio science application: the use of precision Doppler tracking of deep space probes as a detector of low-frequency ${ }^{1}$ gravitational waves (GWs). Precision Doppler experiments were pioneered by Vessot, whose GP-A suborbital experiment measured the general relativistic redshift in the earth's static gravitational field [126]. In the deep space GW observations discussed here, the earth and a distant spacecraft are free test masses with the ground-based Doppler tracking system continuously measuring the earth-spacecraft fractional velocity $(2 \Delta v / c=$ $\Delta \nu / \nu_{0}$, with $\Delta \nu$ being the Doppler shift and $\nu_{0}$ being the radio link's carrier frequency). A gravitational wave with strain amplitude $h$ causes perturbations of order $h$ in $\Delta \nu / \nu_{0}$. Unlike other GW detectors, the $\sim 1-10$ AU earth-spacecraft separation makes the detector large compared with millihertz-band gravitational wavelengths. Consequently times-of-flight of the GWs and radio waves through the apparatus are important and impose characteristic signatures of GWs in the observed Doppler time series.

The theory of the (two-way) Doppler GW detector was built up by generalizing the response of so-called one-way Doppler measurements. In one-way tracking, each of two test masses has its own frequency standard. Equipment on one test mass transmits a wave referenced to its frequency standard and a receiver on the other mass estimates the Doppler shift by comparing the frequency of the wave it receives with the frequency of its local standard. ${ }^{2}$ In 1970, Kaufmann [74] calculated the fractional frequency fluctuation caused by GWs on one-way Doppler in the context of proposed earth-based GW detectors using the Mössbauer effect. In 1971, Anderson [2] commented on $100 \mathrm{~s}$ fluctuations in Mariner 6's Doppler time series with the suggestion that these might be related to resonant-bar events reported at roughly the same time. In 1974, Davies [42] surveyed the prospects for GW detection with deep space probes. He carefully noted the sensitivity advantages of Doppler (as contrasted with ranging), identified several competing error sources, and presented the GW response for two-way Doppler in the special case of GWs incident normal to the earth-spacecraft line. In 1975, Estabrook and Wahlquist [52] derived the general GW response for arbitrary angle-of-

\footnotetext{
1 Gravitational-wave bands conventionally divide based on detector technology [108, 35]: Future extremely-lowfrequency $\left(\sim 10^{-18}\right.$ to $\sim 10^{-15} \mathrm{~Hz}$ ) search programs will be based on mapping the intensity and polarization of the cosmic microwave background; very-low frequency observations $\left(\sim 10^{-9}\right.$ to $\left.\sim 10^{-6} \mathrm{~Hz}\right)$ mostly use pulsar timing observations; low-frequency $\left(\sim 10^{-6}\right.$ to $\left.\sim 10^{-1} \mathrm{~Hz}\right)$ observations currently use Doppler tracking of spacecraft (in the 2020's a laser interferometer in space); high-frequency ( $\sim 1$ to $\left.\sim 10^{4} \mathrm{~Hz}\right)$ observations involve ground-based laser interferometers or resonant bar detectors. For general reviews see [91, 108, 36].

2 Although conceptually important - and used with excellent success as part of the hydrogen-maser-based suborbital GP-A experiment [126] - one-way Doppler presents a practical problem for precision tracking of deep space probes: Flight-qualified frequency standards for deep space are currently (2015) substantially less stable than ground-based standards. The quality of one-way spacecraft Doppler GW measurements is severely limited by noise in the flight frequency generator. Deep space tracking systems circumvent this by measuring two-way Doppler. In the two-way mode the ground station transmits a radio signal referenced to a high-quality frequency standard. The spacecraft receives this signal and phase-coherently retransmits it to the earth. The transponding process adds noise, but at negligible levels in current observations (see Table 2), and does not require a good oscillator on the spacecraft. The ground station then measures the two-way Doppler shift by comparing the frequency of the received signal against the frequency of a local reference derived from the ground frequency standard.
} 
arrival and for a detector large compared with the GW wavelength (see Section 3) and derived the spectral distribution of Doppler fluctuations due to an isotropic GW background. With colleagues they considered signal and noise transfer functions, the sensitivity of Doppler tracking to GWs (including the prospects for improving it), and the utility of simultaneous tracking of several spacecraft [52, 128, 46, 50, 47]. In 1976, Thorne and Braginsky [109] estimated event rates for low-frequency GW bursts and discussed the prospects for observing these bursts with spacecraft Doppler tracking. The first systematic GW observations with deep-space Doppler tracking were made in the 1980s; those observations - and technical developments in the following two decades resulting in thousand-fold improved GW sensitivity - are discussed below. 


\section{Notation, Acronyms, and Conventions}

Table 1 defines some acronyms used in this article. Since it is always clear from context, I use the same symbol for theoretical statistical quantities (e.g., underlying spectra) and estimates derived from finite data sets (e.g., sample spectra). All power spectra are two-sided.

Table 1: Some acronyms and notation used in this paper.

\begin{tabular}{|c|c|}
\hline Symbol & Meaning \\
\hline acf & autocorrelation function \\
\hline $\mathrm{AMC}$ & Advanced Media Calibration system (see Section 4.3) \\
\hline DOY & day-of-year, i.e., January $20=$ DOY 020 \\
\hline DSN & $\begin{array}{l}\text { Deep Space Network, the NASA/JPL spacecraft communications and tracking } \\
\text { network with antennas at complexes in Australia, Spain, and the United States }\end{array}$ \\
\hline DSS & Deep Space Station (identifier for specific antennas in the DSN, e.g., "DSS 25") \\
\hline$f$ & Fourier frequency \\
\hline FTS & Frequency and Timing $\mathrm{S}$ \\
\hline Ka-band & $\begin{array}{l}\text { a deep space communications frequency } \simeq 32 \mathrm{GHz} \\
\text { (for Cassini, "Ka1" is the downlink referenced to the X-band uplink; "Ka2" is the } \\
\text { downlink referenced to the Ka-band uplink) }\end{array}$ \\
\hline $\mathrm{KaT}$ & $\begin{array}{l}\text { Cassini's "Ka-band Translator" which accepts an uplink Ka-band signal and phase- } \\
\text { coherently translates it to a downlink Ka-band signal for retransmission to earth }\end{array}$ \\
\hline LISA & Laser Interferometer Space Antenna, a future low-frequency GW observatory [24] \\
\hline $\begin{array}{l}\text { one-way } \\
\text { pdf }\end{array}$ & $\begin{array}{l}\text { Doppler tracking where the downlink is referenced to an oscillator on the spacecraft } \\
\text { probability density function [88] }\end{array}$ \\
\hline S-band & a deep space communications frequency $\simeq 2.3 \mathrm{GHz}$ \\
\hline scintillation & phase fluctuation due to wave propagation in a random medium $[106,79,40,99,71]$ \\
\hline SEP angle & sun-earth-probe angle, the apparent solar elongation angle of the spacecraft \\
\hline$S_{x}(f)$ & power spectrum of $x(t)$, i.e., Fourier transform of $\langle x(t) x(t+\tau)\rangle[73,89]$ \\
\hline & duration of an observation \\
\hline$T_{2}$ & two-way light time between earth and spacecraft \\
\hline three-way & $\begin{array}{l}\text { Doppler tracking where the received downlink is coherently referenced to an uplink } \\
\text { signal from another station }\end{array}$ \\
\hline two-way & Doppler tracking where the downlink is coherently referenced to the uplink signal \\
\hline X-band & \\
\hline$x * y$ & avolution of time series $x(t)$ and $y(t)[34]$ \\
\hline$y(t)$ & generic time series of fractional frequency, $\Delta \nu / \nu_{0}[23]$ \\
\hline & $\begin{array}{l}\left(y_{2}(t): \text { time series of two-way Doppler fractional frequency) }\right. \\
\left(y_{3}(t) \text { : time series of three-way Doppler fractional frequency) }\right.\end{array}$ \\
\hline$\nu$ & radio frequency \\
\hline$\sigma_{y}($ & Allan deviation, fractional frequency stability at integration time $\tau$ [23] \\
\hline & angle between GW propagation direction and earth-spacecraft vector; $\mu=\cos (\theta)$ \\
\hline
\end{tabular}




\section{Gravitational Wave Signal Response}

The theory of spacecraft Doppler tracking as a GW detector was developed by Estabrook and Wahlquist [52]. Briefly, consider the earth and a spacecraft as separated test masses, at rest with respect to one another and separated by distance $L=c T_{2} / 2$, where $T_{2}$ is the two-way light time (light time from the earth to the spacecraft and back). A ground station continuously transmits a nearly monochromatic microwave signal (center frequency $\nu_{0}$ ) to the spacecraft. This signal is coherently transponded by the distant spacecraft and sent back to the earth. The ground station compares the frequency of the signal which it is transmitting with the frequency of the signal it is receiving. The two-way fractional frequency fluctuation is $y_{2}(t)=\left[\nu\left(t-T_{2}\right)-\nu(t)\right] / \nu_{0}$, where $\nu(t)$ is the frequency of the actual transmitted signal. In this way the Doppler tracking system measures the relative dimensionless velocity of the earth and spacecraft: $2 \Delta v / c=\Delta \nu / \nu_{0}$. In an idealized system (no noise, no systematic effects, no gravitational radiation), this time series would be zero.

A GW incident on this system causes perturbations in the Doppler frequency time series. The gravitational-wave response $y_{2}^{\mathrm{gw}}(t)$ of a two-way Doppler system excited by a transverse, traceless plane gravitational wave [84] having unit wavevector $\hat{k}$ is [52]

$$
y_{2}^{\mathrm{gw}}(t)=\frac{\mu-1}{2} \bar{\Psi}(t)-\mu \bar{\Psi}\left(t-\frac{1+\mu}{2} T_{2}\right)+\frac{1+\mu}{2} \bar{\Psi}\left(t-T_{2}\right),
$$

where $\mu=\hat{k} \cdot \hat{n}, \hat{n}$ is a unit vector from the earth to the spacecraft, $\bar{\Psi}(t)=(\hat{n} \cdot \mathbf{h}(t) \cdot \hat{n}) /\left(1-(\hat{k} \cdot \hat{n})^{2}\right)$, and $\mathbf{h}(t)$ is the first order metric perturbation at the earth. (Here $\bar{\Psi}$ is distinguished from the $\Psi$ used to analyze the LISA detector $[16,51,119,120]: \Psi=(1 / 2) \bar{\Psi}$.) The GW amplitude at the earth is $\mathbf{h}(\mathbf{t})=\left[\mathbf{h}_{+}(\mathbf{t}) \mathbf{e}_{+}+\mathbf{h}_{\times}(\mathbf{t}) \mathbf{e}_{\times}\right]$, where the 3 -tensors $\mathbf{e}_{+}$and $\mathbf{e}_{\times}$are transverse to $\hat{k}$ and, with respect to an orthonormal $(\hat{i}, \hat{j}, \hat{k})$ propagation frame, have components

$$
\mathbf{e}_{+}=\left(\begin{array}{rrr}
1 & 0 & 0 \\
0 & -1 & 0 \\
0 & 0 & 0
\end{array}\right), \quad \mathbf{e}_{\times}=\left(\begin{array}{lll}
0 & 1 & 0 \\
1 & 0 & 0 \\
0 & 0 & 0
\end{array}\right)
$$

(If general relativity and a transverse traceless perturbation are not assumed, the amplitude of the three-pulse response for a general tensor metric perturbation is given in [57].)

The Doppler responds to a projection of the time-dependent wave metric, in general producing a "three-pulse" response to a pulse of incident gravitational radiation: one event due to buffeting of the earth by the GW, one event due to buffeting of the spacecraft by the GW, and a third event in which the original earth buffeting is transponded a two-way light time later. The amplitudes and locations of the pulses depend on the arrival direction of the GW with respect to the earthspacecraft line, the two-way light time, and the wave's polarization state. From Eq. (1) the sum of the three pulses is zero. Since the detector response depends both on the spacecraft-earthGW geometry $\left(T_{2}, \mu\right)$ and the wave properties (Fourier frequency content, polarization state) its distinctive three-pulse signature plays an important role in distinguishing candidate signals from competing noises. Figure 1 shows this three pulse response in schematic form.

In the special case of the long-wavelength limit (LWL, where the Fourier frequencies of the GW signal are $\left.\ll 1 / T_{2}\right)$, the gravitational wave can be expanded in terms of spatial derivatives. Equation (1) then gives the LWL response for two-way Doppler tracking:

$$
y_{2}^{\mathrm{gw}, \mathrm{LWL}}(t) \rightarrow \frac{T_{2}}{2}\left(\mu^{2}-1\right) \bar{\Psi}^{\prime}(t)
$$

In this limit the three-pulses overlap in the tracking record causing partial cancellation, loss of signature, and loss of signal response. In the opposite limit, wave periods $\ll T_{2}$, the full three 

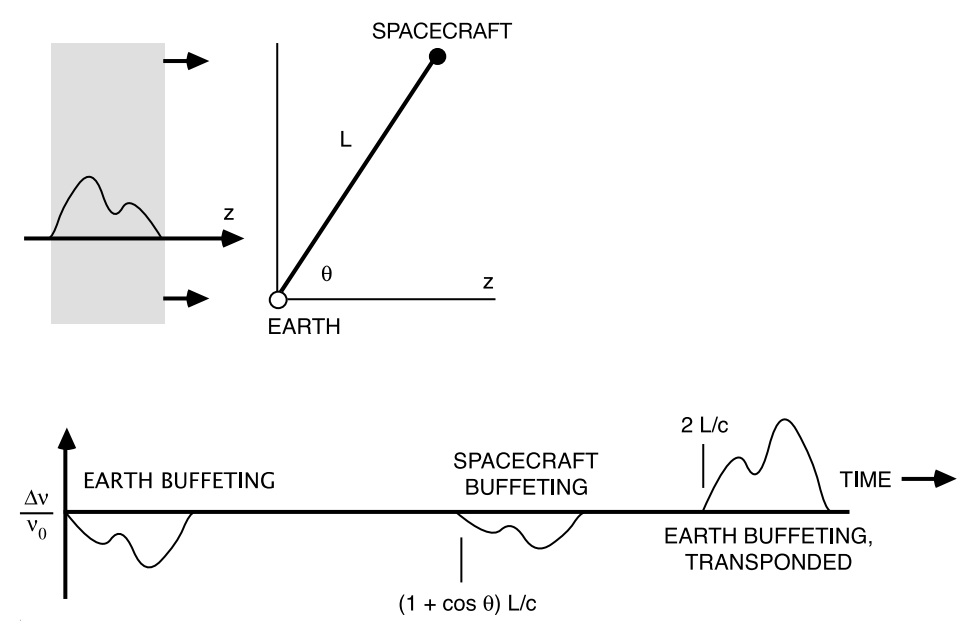

Figure 1: Schematic response of two-way Doppler tracking to a GW. The Doppler exhibits three pulses having amplitudes and relative locations which depend on the GW arrival direction, the two-way light time, and the wave's strain amplitude and polarization state. The sum of the three pulses is zero, so the pulses overlap and partially cancel when the characteristic time of the GW pulse is comparable to or larger than the light time between the earth and spacecraft.

pulse character [Eq. (1)] is expressed in the Doppler time series. Figure 2 shows the spectral response of a Doppler tracking system to sinusoidal GW signals from two specific directions and the average response from sources distributed isotropically on the celestial sphere. Because Figure 2 plots the transfer function to the spectral power, the dependence at low-frequency is $\propto f^{2}$. The algorithm used to average over GW polarization states in Figure 2 is described in $[18,16]$. A related discussion (how to infer GW amplitudes, $h$ - or limits to $h$ - from measurements of $y_{2}$ when the signal direction and polarization state are unknown) is in [18]. The LWL of the three-pulse GW response has been used to analyze the GW response of ground-based Michelson gravitational wave interferometers [47]. The three-pulse response can also be constructed using the formalism of time-delay interferometry, the method LISA will use to cancel laser phase noise in an unequal-arm spaceborne GW detector (see Section 8). The formalism has also been applied to analysis of other spaceborne detector geometries, for example the candidate linear array, SyZyGy [49].

In a practical GW observation spanning 20-40 days, the earth-spacecraft distance and the orientation of the earth-spacecraft vector on the celestial sphere change (typically slowly) with time. This modifies the idealized GW response (it is not strictly time-shift invariant) and has practical consequences in searches for long-lived signals (see Section 5.7).

To summarize the Doppler signal response:

1. GW signals are observed in the Doppler tracking time series through the three pulse response [Eq. (1)].

2. The response depends on the two-way light time $T_{2}$, the cosine of the angle between the GW wavevector and unit vector from the earth to the spacecraft, and GW properties (Fourier content and polarization state) (Eq. (1) and the expression for $\bar{\Psi}(t)$ ).

3. The GW response is not in general time-shift invariant if $T_{2}$ or $\mu$ change during the time of observation.

4. The GW response is a high-pass filter: In the long-wavelength limit (frequencies $\ll 1 / T_{2}$ ), the response is attenuated due to pulse overlap and cancellation (see Figure 2). 


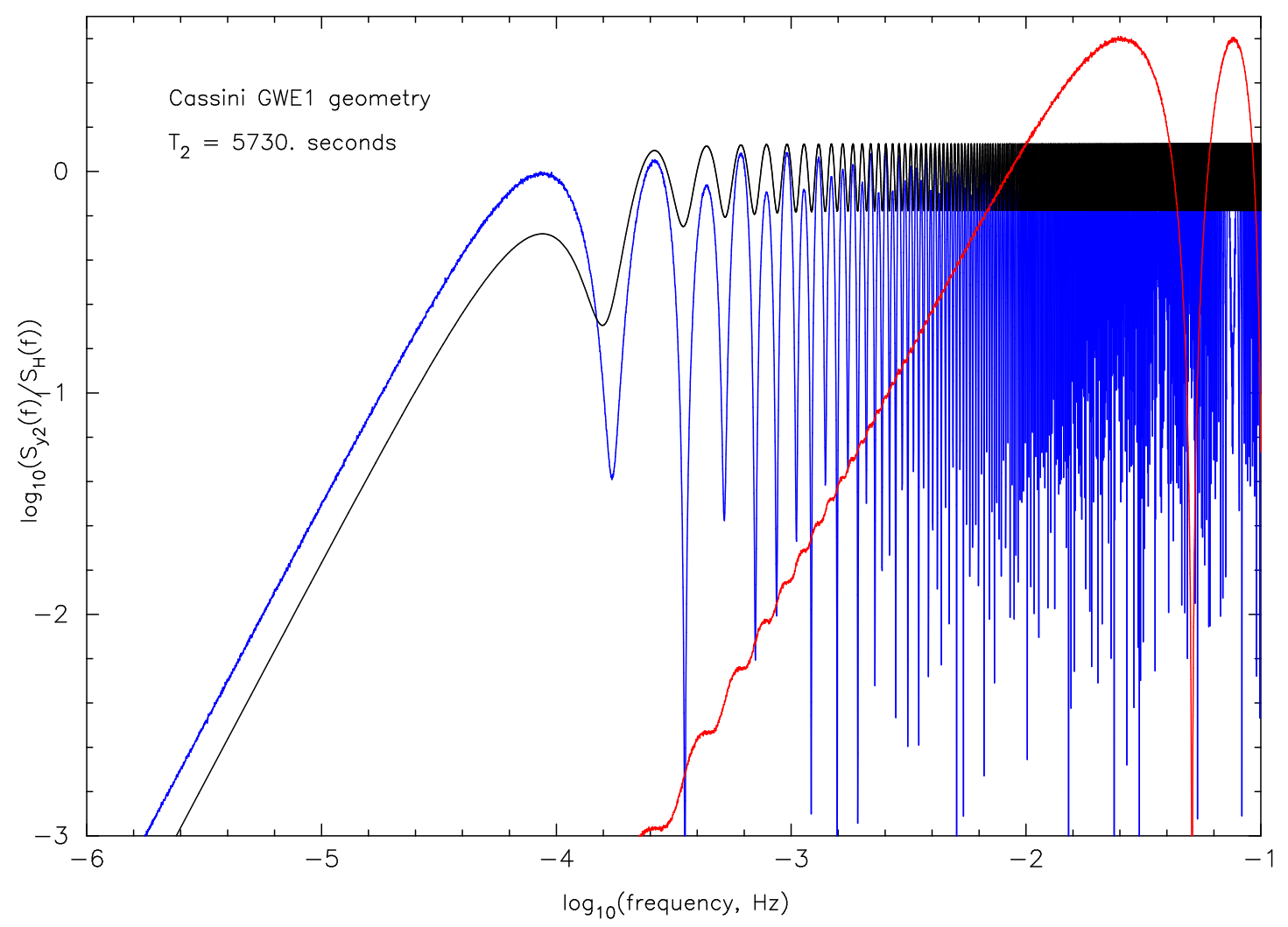

Figure 2: Polarization-averaged power response of the Doppler system to a gravitational wave signal as a function of Fourier frequency. Earth-spacecraft geometry for Cassini's gravity wave observations in 20002001 has been used. Blue: response from the direction of Virgo $(\mu \simeq 0.104)$. Black: response to randomly polarized sources distributed isotropically on the celestial sphere. Red: response from the direction of the galactic center $(\mu \simeq 0.9932)$. Note that in the case of waves from the galactic center, the three-pulse response function ([52] and Eq. (1)) for the Cassini geometry is dominated by two pulses separated by only about $0.00034 T_{2} \simeq 20 \mathrm{~s}$. This gives rise to the strong low-frequency suppression and the approximate $\sin ^{2}$ modulation for the Cassini GWE1 geometry and GWs from the galactic center. 


\section{Apparatus and Principal Noise Sources}

The detector consists of the earth and a spacecraft as separated test masses, electromagneticallytracked using a precision Doppler system. The ground stations for the Doppler system are the antennas of the NASA/JPL Deep Space Network (DSN). Figure 3 shows DSS 25, the high-precision tracking station used in the Cassini gravitational-wave observations and other Cassini radio science investigations.

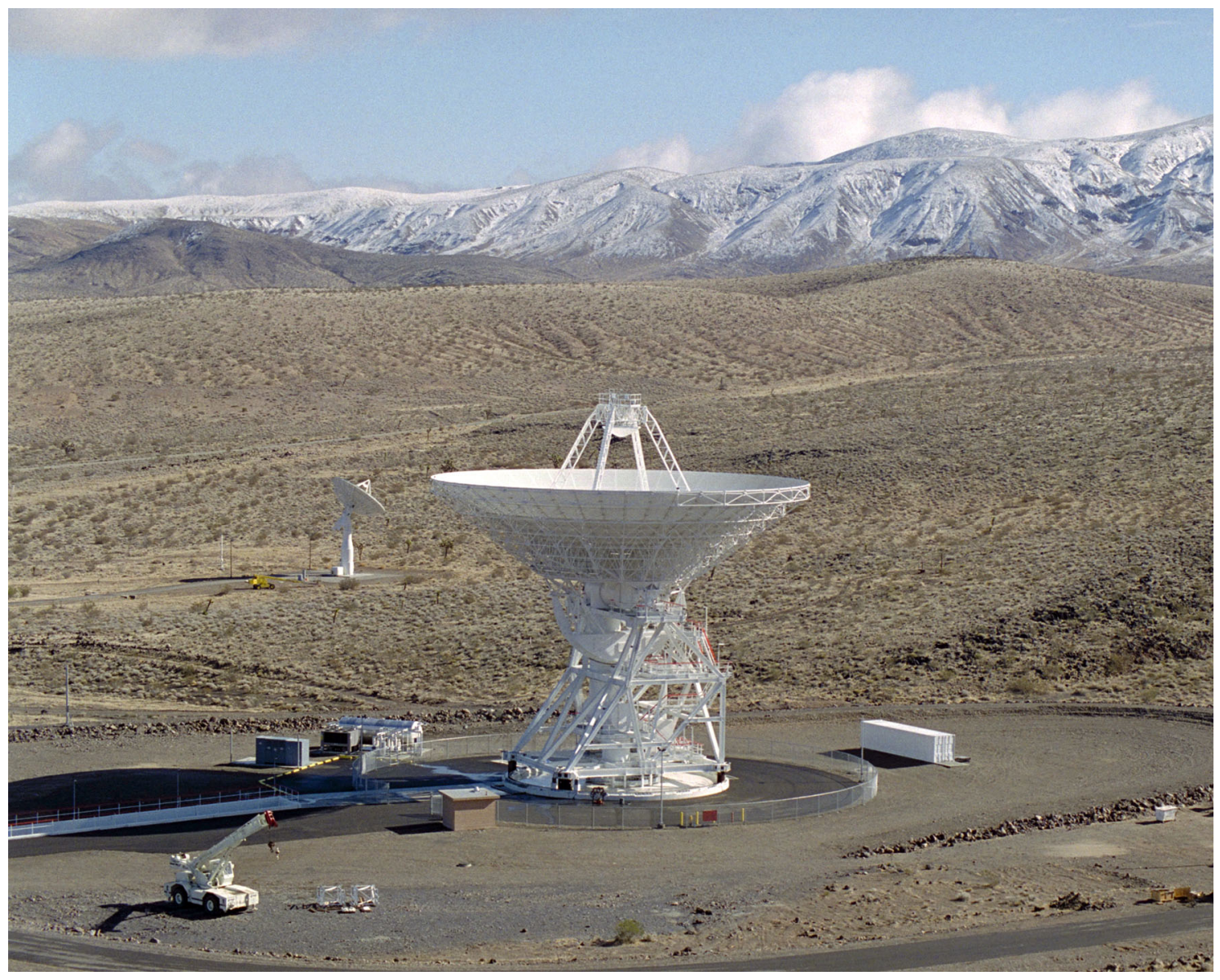

Figure 3: DSS 25, a 34-m beam-waveguide antenna, shown here in the stowed position. DSS 25 is one antenna in the NASA/JPL Goldstone Deep Space Communications Complex near Barstow, CA, U.S.A. It has special instrumentation (Ka-band up- and downlink and advanced tropospheric calibration capability) which enable particularly good quality Doppler observations.

Figure 4 shows an example of the other part of the Doppler system. This is the Cassini spacecraft during ground tests. (Reference [37] gives a popular discussion of the Cassini mission, the spacecraft, and its instrumentation.) The Doppler system is shown functionally in Figure 5: A precision frequency standard from the Frequency and Timing Subsystem (FTS) provides the frequency reference to both the transmitter and receiver chains. On the transmitter side, the so-called exciter produces a near-monochromatic signal, referenced to the FTS signal but at the desired transmit frequency. This is amplified by the transmitter (with a closed-loop feedback system around the power amplifier to ensure frequency stability is not degraded) and routed via waveguide 
to the transmitter feedhorn in the basement of the antenna. (To correct for aberration the Kaband transmit feed horn is on a table which is articulated in the horizontal plane. This allows the Ka-band transmitted beam to be pointed correctly relative to the received beam. The X-band feed is common to both the transmit and receive chains.) In a beam waveguide antenna the transmitted beam is reflected off of six mirrors within the antenna up to the subreflector (near the prime focus), then back to the main dish and out to the spacecraft (passing first through the troposphere, ionosphere, and solar wind). When the signal is received at the spacecraft it is amplified and phase-coherently re-transmitted to the earth. The received beam bounces off the main reflector to the subreflector and then, via mirrors and dichroic plates, to the receiver feed horn in the antenna basement. The received signal is downconverted to an intermediate frequency where it is digitized. The digital samples are processed to tune out the (very predictable) gross Doppler shift, and reduce the bandwidth of the samples. For GW operations, the bandwidth of the pre-detection data is typically reduced to $1 \mathrm{kHz}$, and those data are recorded to disk along with the tuning information. The phase of the signal is detected in software and, using the tuning information, the received sky frequency is reconstructed. This and the known frequency of the transmitted signal are used to compute the Doppler time series. Removal of the orbital signature and correction for charged particle and tropospheric scintillation gives Doppler residuals, which are used in subsequent processing steps to search for GWs (or for other radio science objectives [29, 131, 78, 22]).

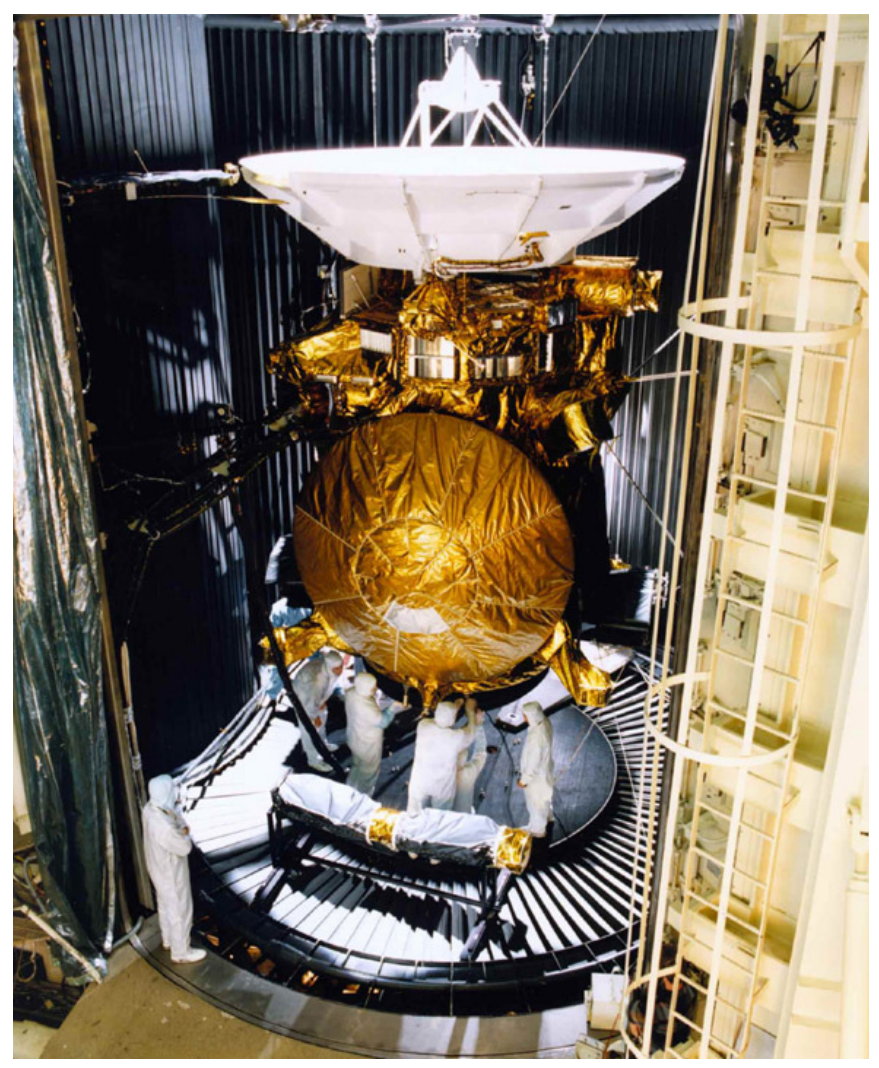

Figure 4: The Cassini spacecraft during pre-launch testing. Reference [37] gives a popular discussion of the Cassini mission, the spacecraft, and its instrumentation (photograph courtesy NASA/JPL-Caltech).

Of course this cannot be done without introducing noise. The following Sections 4.1-4.11 


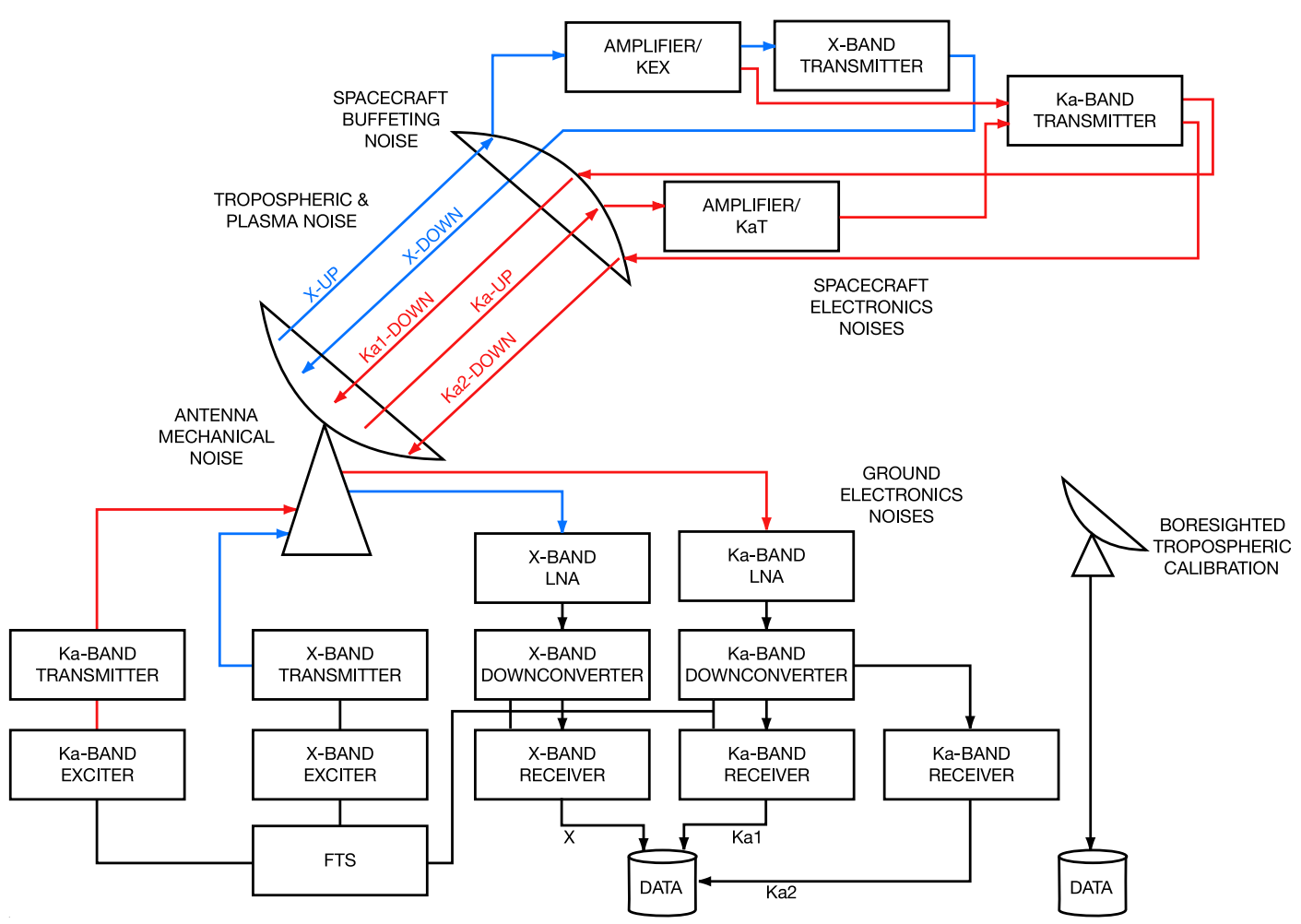

Figure 5: Conceptual sketch of signal flow for two-way Cassini observations, with emphasis on showing which links are affected by specific noise sources. For example, spacecraft buffeting and the frequency and timing subsystem (FTS) are common to all Doppler links. The Ka-band translator (KaT) affects only the Ka2 downlink, while the conventional transponder (KEX) affects both the X-downlink and the Ka1 downlink, etc.

summarize the principal noises, their spectra or Allan deviations ${ }^{3}$, and their transfer functions to the two-way Doppler time series.

\subsection{Frequency standard noise}

In two-way Doppler coherence is maintained by the frequency standard to which the up- and downlinks are referenced. Thus noise introduced by the frequency standard is of particular importance. Figure 6 shows fractional frequency stability as a function of integration time for several frequency standard technologies. In Cassini-era observations noise in the frequency and timing system (FTS) contributed less than $10^{-15}$ at $1000 \mathrm{~s}$ and, although fundamental, is not the leading noise source at the current level of sensitivity. (FTS stability required for future Doppler experiments is discussed in Section 7.)

FTS noise enters the two-way Doppler time series via the transfer function $[52,46,125]$ $y^{\mathrm{FTS}}(t) *\left[\delta(t)-\delta\left(t-T_{2}\right)\right]$. The transfer functions of this and other principal noises are illus-

\footnotetext{
${ }^{3}$ Noises are characterized in the time domain by Allan deviation, $\sigma_{y}(\tau)$, or in the frequency domain by the power spectra of fractional frequency fluctuations, $S_{y}(f)$. These are related by

where $S_{y}(f)$ is the two-sided spectrum [23].

$$
\sigma_{y}^{2}(\tau)=4 \int_{0}^{\infty} \frac{S_{y}(f) \sin ^{4}(\pi \tau f)}{(\pi \tau f)^{2}} d f
$$
}




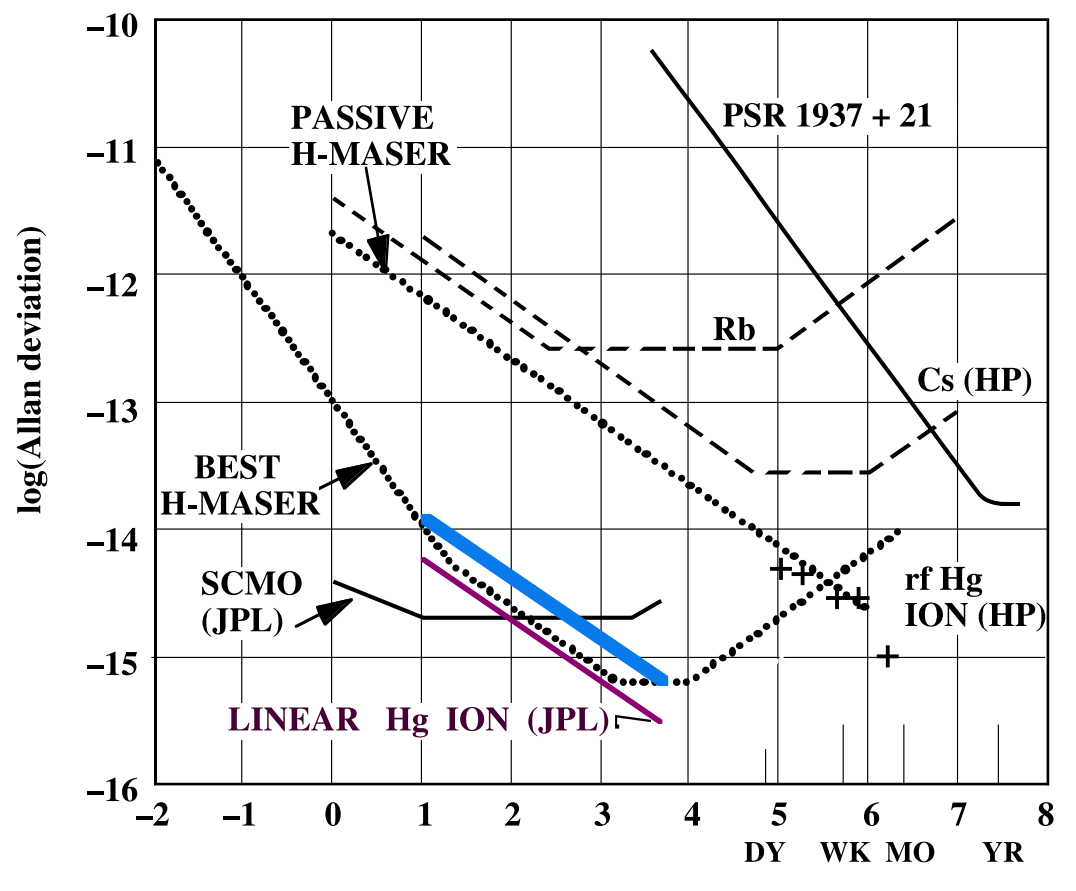

$\log$ (integration time, seconds)

Figure 6: Allan deviation (square root of Allan variance [23]) as a function of integration time for several frequency and timing technologies in the Cassini-era. The technology used in precision Doppler observations for GW searches with Cassini has $\sigma_{y}\left(1000 \mathrm{~s}\right.$ ) less than $10^{-15}$. (Figure courtesy of Lute Maleki; see also references $[10,11,22]$.)

trated schematically in Figure 7. (An example of the FTS transfer function using real data is shown in Figure 8. Although the stability of the ground frequency standard is excellent, for a few days at the start of the first Cassini Gravitational Wave Experiment there was an intermittent problem with an FTS distribution amplifier at the Goldstone complex. The effect was to introduce isolated, fairly large, and very short glitches into the frequency reference for both the transmitter and the receiver. This produced characteristic anticorrelated glitches, separated by a two-way light time, in both the X- and Ka-band two-way Doppler time series; see Figure 8)

\subsection{Plasma scintillation noise}

The radio waves of the Doppler system pass through three irregular media: the troposphere, the ionosphere, and the solar wind. ${ }^{4}$ Irregularities in the solar wind and ionospheric plasmas cause irregularities in the refractive index. The refractive index fluctuations $\delta n$ for a cold unmagnetized plasma are $-\lambda^{2} r_{e} \delta n_{e} /(2 \pi)$ and the phase perturbation is $-\int \lambda r_{e} \delta n_{e} d z$, where $\lambda$ is the wavelength, $r_{e}$ is the classical electron radius, and $\delta n_{e}$ is the electron density fluctuation along the line of sight $z$. These phase perturbations mimic time-varying distance changes (thus velocity errors) and so are a noise source in precision Doppler experiments. The transfer function of plasma phase scintillation to two-way Doppler is shown schematically in Figure 7. A solar wind plasma blob at a distance $x$ from the earth (producing a one-way fractional frequency fluctuation time series $y^{\mathrm{sw}}$ ) and an ionospheric plasma blob at negligible light time from the ground station (with one-way time series

\footnotetext{
4 There is a large literature on wave propagation through random media. Excellent general references for radiowave propagation observations include [106, 79, 40, 99, 71].
} 


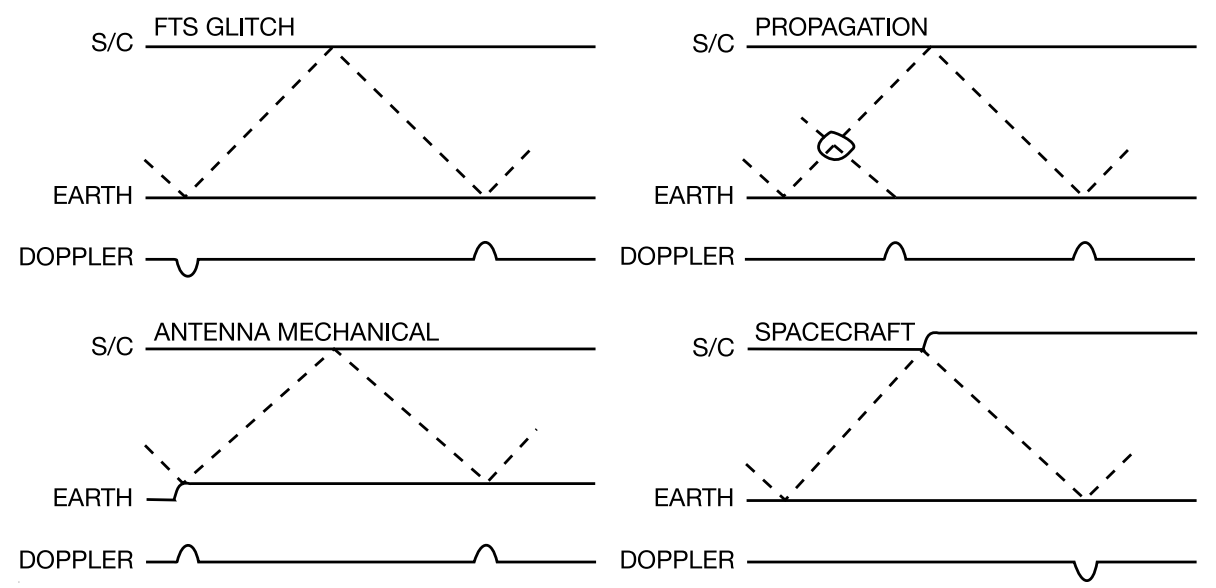

Figure 7: Schematic transfer functions of noises to the two-way Doppler link, adapted from [46, 125]. For each type of disturbance a separate diagram (space vertically, time horizontally) is shown. Radio waves propagate continuously up to and down from the spacecraft; some of these are represented as the dashed lines to illustrate the indicated Doppler frequency perturbations. For example, a momentary glitch in the FTS affects the frequency reference for both the receiver and the transmitter. This shows up as an immediate effect in the received Doppler (difference between the transmitted and received frequency). Because the glitch also affects the transmitted frequency, it shows up again - but with the opposite sense - in the Doppler after a two-way light time. These various noise responses contrast with the three-pulse GW response, shown in Figure 1; these differences are exploited in the signal processing.

$\left.y^{\text {ion }}\right)$ produce two-way time series $y^{\mathrm{sw}}(t) *\left[\delta(t)+\delta\left(t-T_{2}+2 x / c\right)\right]$ and $y^{\text {ion }} *\left[\delta(t)+\delta\left(t-T_{2}\right)\right]$, respectively.

Plasma scintillation is mostly a statistical contribution to variability in the two-way Doppler time series. As such it can be seen in the autocorrelation function (acf) of the Doppler time series. Examples of S-band correlation functions which peak at $\tau \simeq T_{2}$ (presumably ionospheric scintillation) and $\tau<T_{2}$ (localized solar wind scintillation ${ }^{5}$ ) are shown in [9]. Occasionally, however, large time-localized plasma events can be seen in the raw time series. Figure 9 shows an example in Cassini data taken at DSS 25 on 2003 DOY 324. The top panel shows the time series of the two-way X-band, with two discrete events observed near 10:20 and 10:40 ground received time, echoed with positive correlation at about the two way light time. The middle panel is the time series of X-(880/3344) Ka1, which isolates the downlink plasma (and cancels nondispersive processes such as FTS noise, tropospheric noise, antenna mechanical noise, and gravitational waves; see Section 4.6). This indicates that the large events observed in the upper panel are due to plasma scintillation. The lower panel shows the acf of the two-way Doppler time series, $\left\langle y_{2}(t) y_{2}(t+\tau)\right\rangle$. The arrow marks the two-way light time. The acf peaks slightly earlier than $T_{2}$, indicating that the features observed in the other panels are caused by near-earth plasma.

Figure 10 summarizes the magnitude of the effect of plasma scintillation, tropospheric scintillation, and antenna mechanical noise (the last two discussed below) on the stability of a Doppler tracking system $[46,9,10,11,12,22]$. Shown in red are data and model curves for plasma phase scintillation: Circles are S-band (frequency $\simeq 2.3 \mathrm{GHz}$ ) observations taken in the ecliptic using the Viking orbiters spacecraft taken over a wide range of sun-earth-spacecraft (SEP) angles [131, 21]; crosses are $\mathrm{X}$-band (frequency $\simeq 8.4 \mathrm{GHz}$ ) taken near the antisolar direction using the Cassini spacecraft $[19,22]$. Clearly plasma scintillation minimizes for observations near the antisolar di-

\footnotetext{
${ }^{5}$ A particularly well-defined example of a spatially-localized solar wind scattering region when Cassini's line-ofsight was close to the sun, thus $\tau$ substantially smaller than $T_{2}$, is shown in [98]
} 

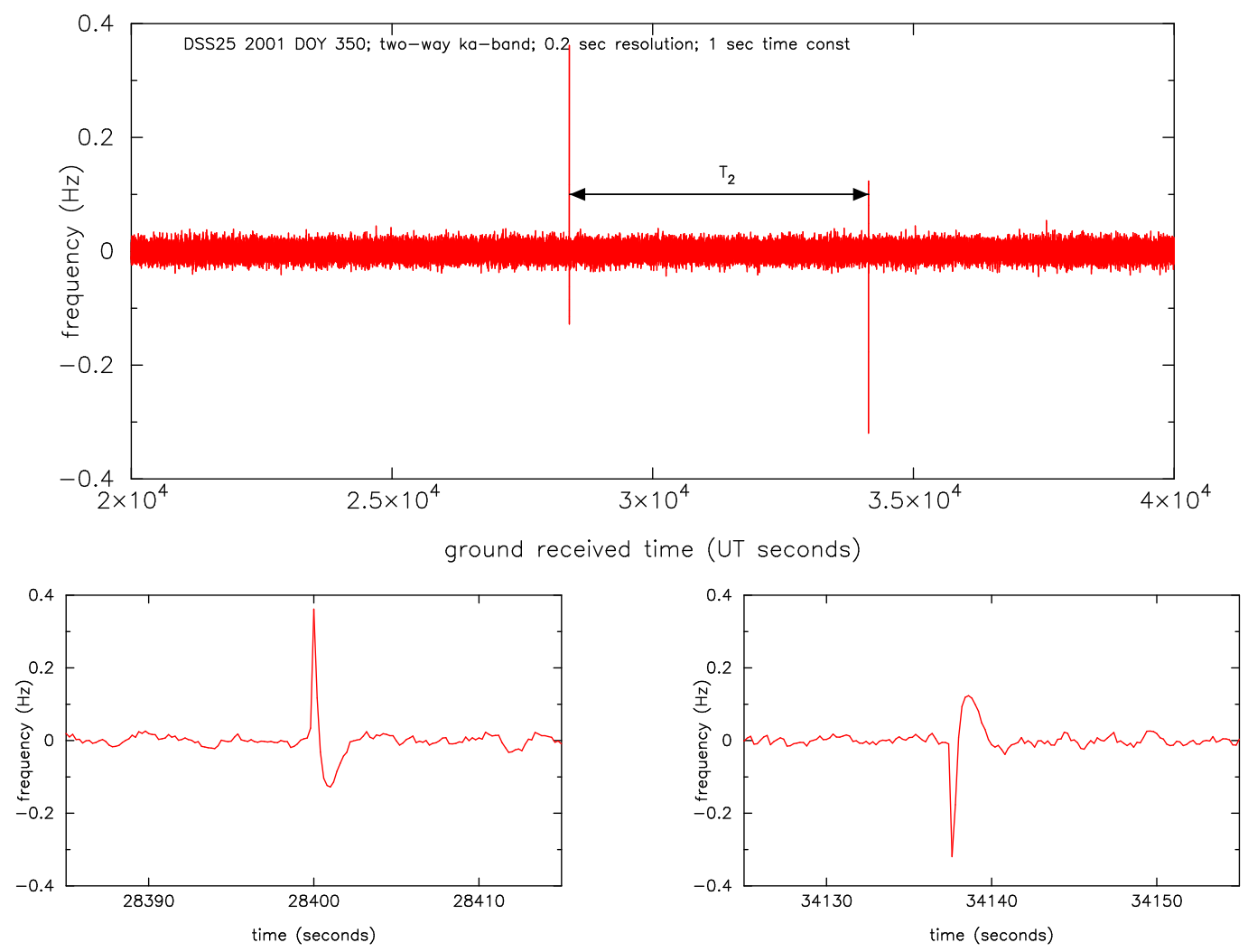

Figure 8: Time series of Cassini two-way Ka-band frequency residuals from a DSS 25 track on 2001 DOY 350. The data are sampled at $0.2 \mathrm{~s}$ after being detected with a time constant $\simeq 1 \mathrm{~s}$. At this time resolution, the visual appearance of the time series is dominated by high-frequency noise. Superimposed on this noise are two systematic glitches which were traced to an intermittently-faulty distribution amplifier in the signal chain providing frequency references to the transmitter and receiver. The distribution amplifier fault acts like an FTS glitch and, in the two-way Doppler, appears twice in the time series anticorrelated at the two-way light time (Figure 7). The glitches in the figure are paired with the indicated two-way light time separation $T_{2} \simeq 5737.7 \mathrm{~s}$. The lower panels show blowups of the pair; the glitch waveforms are unresolved (shapes set by the impulse response of the software phase detector) but clearly show the characteristic FTS anticorrelation.

rection. The model curves drawn through the data are described in [21]. (Ionospheric phase scintillation is, of course, included in the data presented in Figure 10. Based on very limited multiple-station observations [21] and on transfer function studies [9], high-elevation-angle plasma noise appears dominated by solar wind rather than ionospheric phase scintillation. In any case, the effect of any plasma scintillation effect can be made small by observing at high enough radio frequencies $[128,46,21]$ or by using multi-link observations [65, 41, 27, 122, 121, 29] to solve-for and remove the plasma scintillation effect.)

\subsection{Tropospheric scintillation noise}

Phase fluctuations also arise from propagation through the neutral atmosphere. Here the so-called dry component of the troposphere is large but fairly steady with the wet component (water vapor fluctuations) being smaller but much more variable [20, 95, 94, 96, 76]. Unlike plasma phase scintillation, propagation in the troposphere is effectively non-dispersive at microwave frequencies [71]. 

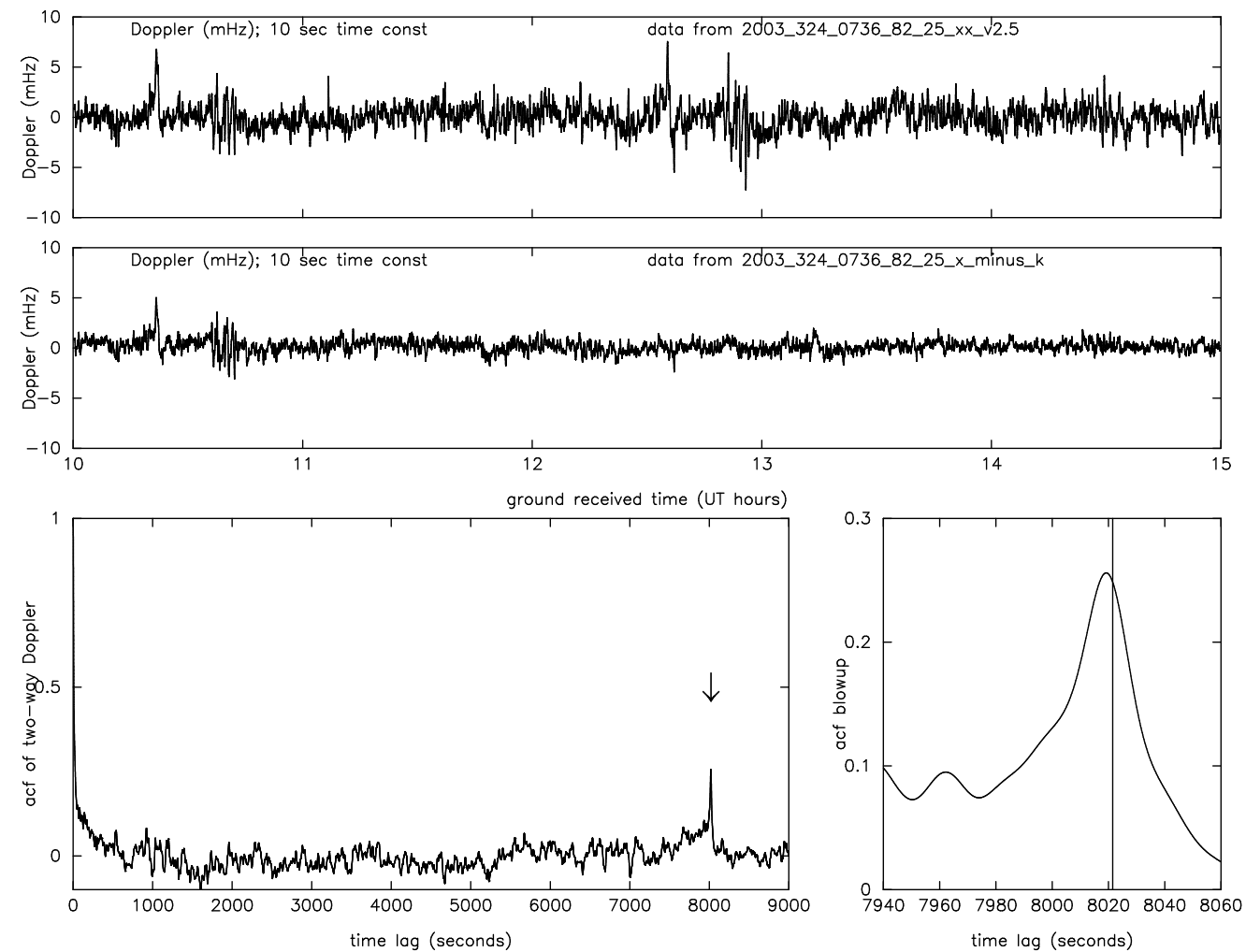

Figure 9: Doppler time series for DSS 25 Cassini track on 2003 DOY 324. Upper panel: time series of the two-way X-band, showing two discrete events at about 10:20 and 10:40 ground received time, echoed about a two way light time, $T_{2}$, later. Middle panel: time series of X-(880/3344) Ka1, which isolates the downlink plasma (and cancels nondispersive noises and signals: FTS, troposphere, antenna mechanical noise, and GWs). This shows that the events observed in the upper panel are due to plasma scintillation. Lower panel: acf of the two-way Doppler time series. The arrow marks the two-way light time. The lower right panel is a blow-up of the acf near the two-way light time (indicated by the vertical line). The acf peaks at lags slightly smaller than $T_{2} \simeq 8021.5 \mathrm{~s}$, indicating that the features observed in the upper panels are caused by near-earth plasma. 


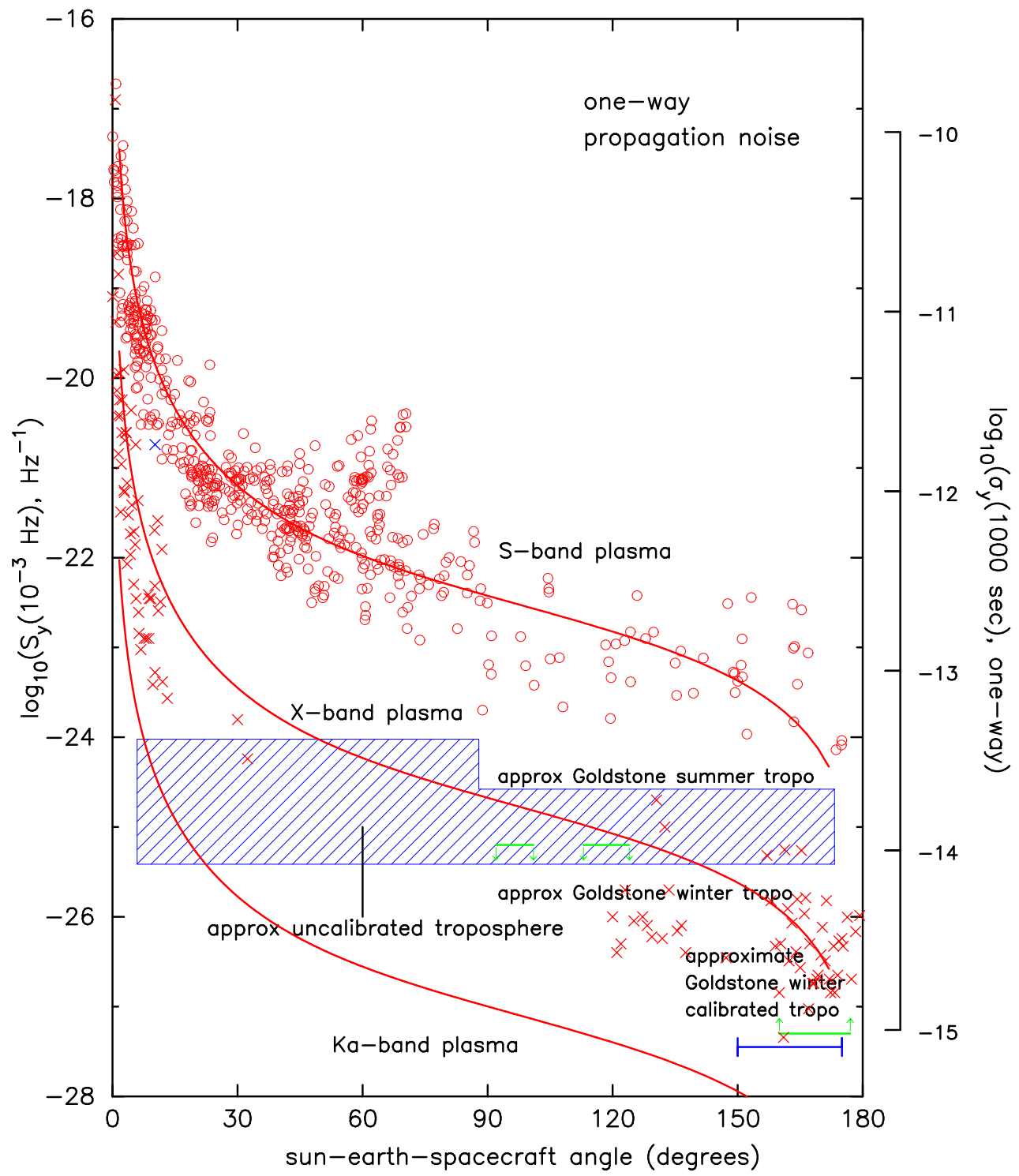

Figure 10: Summary of propagation and antenna mechanical noises as functions of sun-earth-spacecraft angle (updated from [22], reproduced by permission of the American Geophysical Union; see also [131, 21, $65,9,10,11,122,121])$. Left axis: spectral density of fractional frequency fluctuations at $f=0.001 \mathrm{~Hz}$. Right axis: fractional frequency fluctuation (Allan deviation $\sigma_{y}$ ) at $\tau=1000 \mathrm{~s}$. S-band $\simeq 2.3 \mathrm{GHz} ; \mathrm{X}-$ band $\simeq 8.4 \mathrm{GHz} ;$ Ka-band $\simeq 32 \mathrm{GHz}$. Red curves are for plasma scintillation at the indicated radio frequencies (circles are S-band, more precisely: S-(3/11)X differential frequency fluctuations, data from Viking [131, 21]); crosses are X-band (more precisely X-(880/3344) Ka1 differential frequency fluctuations, from Cassini $[122,11,19,29,121])$. Blue region shows typical uncalibrated tropospheric scintillation levels at a moderate-altitude dry site such as Goldstone, CA, or the National Radio Astronomy Observatory's Very Large Array [20, 75, 76]. Green arrows show upper (for antennas in the DSN "high efficiency" subnetwork, operated under operational but benign conditions) and lower (for DSS 25, near solar opposition) limits to antenna mechanical noise [9, 19, 22]. 
Figure 10 shows the magnitude of the effect: The blue cross-hatched region is the approximate level of uncalibrated tropospheric scintillation at NASA's Goldstone Deep Space Communications Complex [75]. Roughly, tropospheric scintillation is worse in the summer daytime and better on winter nights. Usually its raw magnitude is large compared with, e.g., antenna mechanical noise (discussed below.)

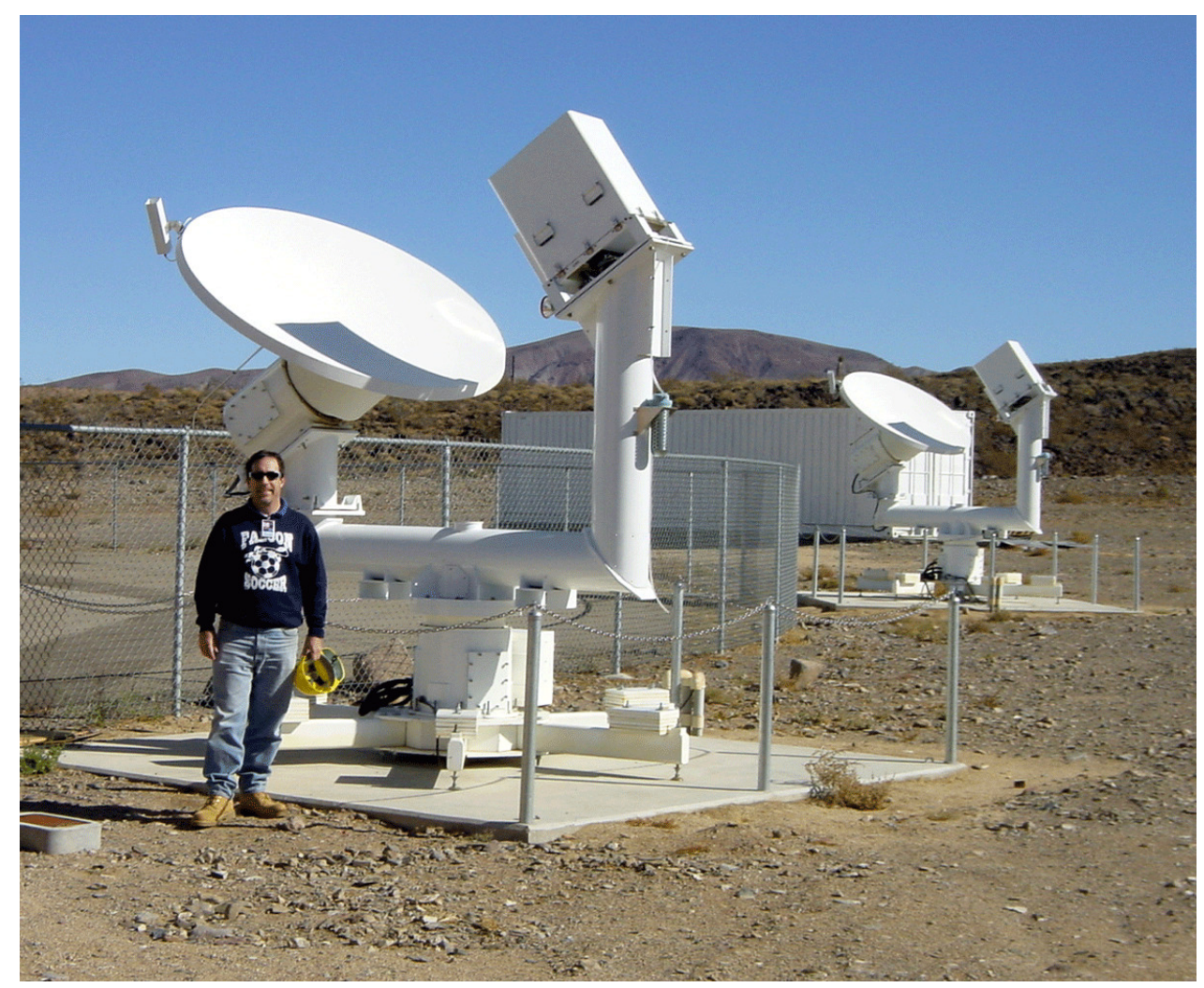

Figure 11: Two water-vapor-radiometer-based Advanced Media Calibration units located near DSS 25, shown here in November 2001. These are used to calibrate tropospheric phase scintillation for the Cassini Gravitational Wave Experiment and other Cassini precision Doppler tracking observations. (Mark Gatti, project manager for the Cassini Radio Science ground system upgrades, is in the foreground.)

Experiments by George Resch and colleagues [95, 94, 96] were influential in showing that suitably boresighted water vapor radiometer measurements could calibrate and remove much of the tropospheric scintillation noise in both radioastronomical and precision spacecraft Doppler tracking observations. A water-vapor-radiometer-based Advanced Media Calibration (AMC) system (Figure 11) was developed and installed near DSS 25 to provide tropospheric corrections for Cassini radio science observations. The AMC system [94, 96, 76] consists of two identical units placed close enough to each other and to DSS 25 that the coherence of the tropospheric signal on the time scales of interest was high in all three time series (see $[10,11]$ for examples of the squared-coherence as a function of Fourier frequency). The AMC calibrations were used successfully in both the Cassini gravitational-wave observations [19] and in relativity and plasma experiments taken near solar conjunction $[29,122,121,22]$. The transfer function of tropospheric scintillation to the two-way Doppler is $y^{\text {tropo }} *\left[\delta(t)+\delta\left(t-T_{2}\right)\right]$. Examples of the cross correlation function of Doppler and the AMC-estimated tropospheric scintillation are shown in $[10,11]$. 


\subsection{Antenna mechanical noise}

Figure 7 shows schematically how mechanical noise in the antenna enters the Doppler. If, for example, the antenna's phase center suddenly moves toward the spacecraft, the received signal is blue shifted, causing an immediate effect in the Doppler. The motion also causes the transmitted signal to be blue shifted; this signal is echoed in the time series a two-way light time later. Early tests by Otoshi and colleagues $[86,87$ indicated that antenna mechanical stability would contribute $\sim 10^{-15}$ for $1000 \mathrm{~s}$ integrations on a 34 -m-class antenna. ${ }^{6}$

Examples of the temporal autocorrelation of a typical Cassini DSS 25 Ka-band up- and downlink tracks taken during the first Cassini GWE campaign in 2001 are shown in [19, 11]. Positive correlation at the two-way light time is characteristic of low-level residual antenna mechanical noise and is observed (with varying level of correlation at $\tau=T_{2}$ ) in all the Cassini DSS 25 GW tracks. Antenna mechanical noise in this band $\left(\simeq 10^{-4}-10^{-1} \mathrm{~Hz}\right)$ is thought to be caused by high-spatial-frequency irregularities in the azimuth ring on which the antenna rolls, wind loading of the main dish, and uncorrected dish sag as the elevation angle changes. In addition to this lowlevel statistical antenna mechanical noise, discrete events positively correlated at the two-way light time and large enough to be visible by eye in the time series are (rarely) observed in operational tracks [11]. Figure 12 shows an example (Cassini tracked by DSS 25 on 2001 DOY 330). The upper panel shows two-way Ka-band Doppler residuals with approximately $10 \mathrm{~s}$ time resolution. The middle panel shows the time series of X-(880/3344) Ka1, i.e., essentially the X-band plasma on the downlink, indicating the low level of plasma noise on this day. The AMC data (not plotted here) similarly show low tropospheric noise. The event at about 07:30 UT is echoed about a twoway light time later, and may be due to gusting wind on this day (another candidate pair is at about 09:45 UT and a two-way light time later). The lower panel shows the autocorrelation of the two-way Ka-band data, peaking at $T_{2}$.

At lower Fourier frequencies (less than about $10^{-4} \mathrm{~Hz}$ ) the apparatus operates in the LWL and the signature of antenna mechanical noise is lost [19]. At these low frequencies aggregate antenna mechanical noise is probably composed both of approximately random processes (e.g., atmospheric pressure loading of the station [81, 123, 38], differential thermal expansion of the structure [100]) and of low-level quasi-deterministic processes (e.g., low-spatial-frequency imperfections in the antenna's azimuth track, systematic errors in subreflector focusing, etc.). Thermal processes (e.g., response of the structure to $\simeq 10 \mathrm{~K}$ temperature variations during a track) can plausibly produce only several millimeters of radio path length variation. The subreflector is continuously repositioned to approximately compensate for elevation-angle dependent antenna distortions; systematic errors in this focusing at the several millimeter level over the course of a track are not unreasonable. Additionally, there are systematic low- and high-spatial-frequency height variations, $\simeq 6 \mathrm{~mm}$ peakto-peak, in the azimuth track which will cause path-length variability. Independently determined VLBI error budgets (omitting components due to radio source structure, uncalibrated troposphere, and charged particle scintillation which are not common with Cassini-class Doppler tracking observations) are believed dominated by station position and slowly-varying antenna mechanical noises. These account for $\simeq 1.3 \mathrm{~cm}$ rms path delay [104], occur on time scales $\simeq 10^{5}-10^{6} \mathrm{~s}$, and correspond to fractional frequency fluctuations $\simeq 10^{-15}$ or smaller.

\subsection{Ground electronics noise}

The DSN ground electronics have been carefully designed to minimize phase/frequency noise and produce only a small contribution to the overall error budget. (Here I distinguish this noise from the

\footnotetext{
6 The mechanical stability of the DSN's 70-m antennas has not been systematically studied. A few observations done with Cassini in 2003 suggest mechanical noise of the 70-m antennas is substantially larger than for the 34-m beam-waveguide antennas.
} 

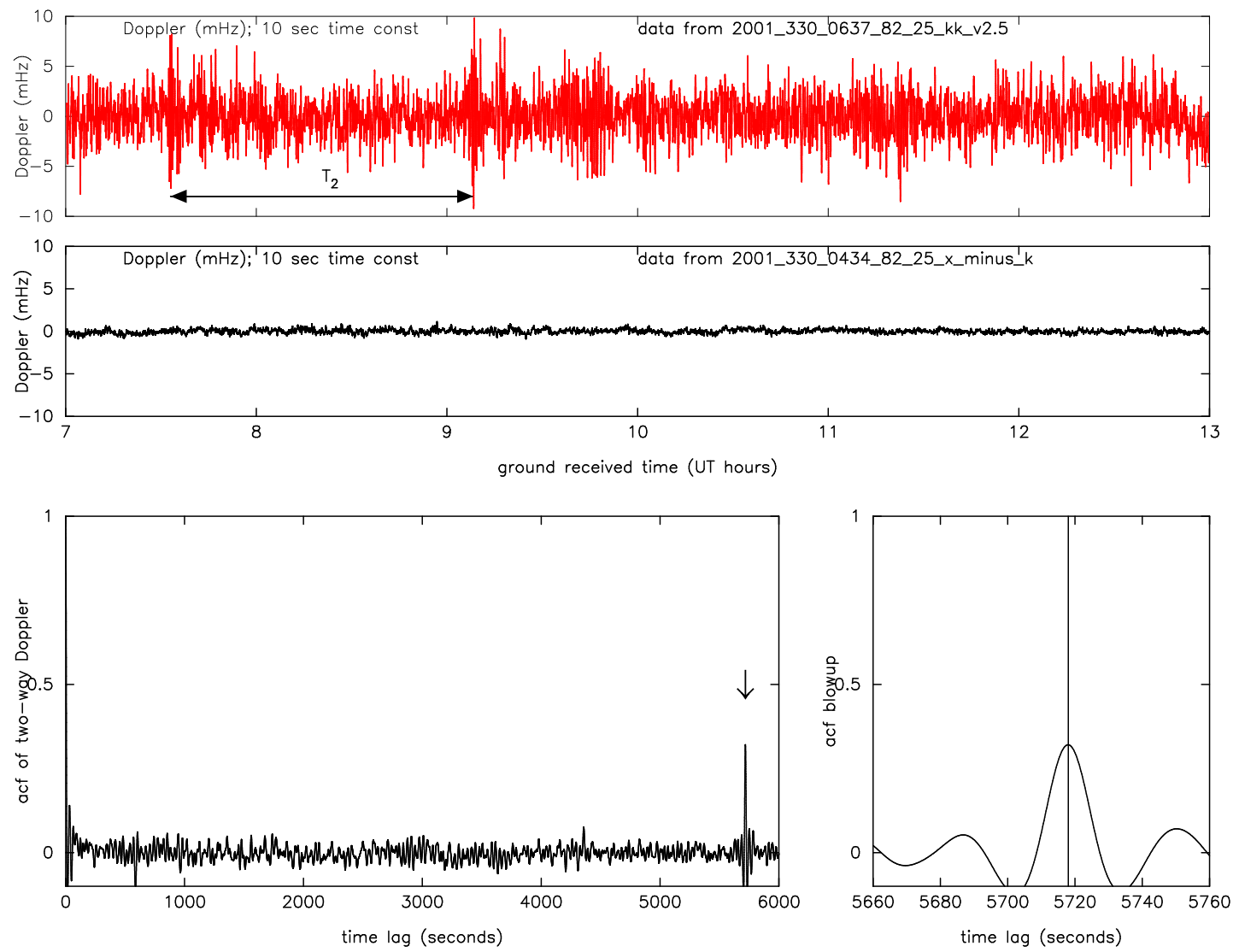

Figure 12: Doppler time series for DSS 25 Cassini track on 2001 DOY 330, showing an antenna mechanical noise event. Upper panel: time series of the two-way Ka-band, with approximately $10 \mathrm{~s}$ time resolution. The middle panel shows the time series of X-(880/3344) Ka1, i.e., essentially the X-band plasma on the downlink, indicating the low level of plasma noise on this day. The AMC data similarly show low tropospheric noise. The event at about 07:30 is echoed about a two-way light time later, and may be due to gusting wind on this day (another candidate pair is at about 09:45 and a two-way light time later). The lower left panel shows the autocorrelation of the two-way Ka-band data, peaking at $T_{2} \simeq 5717.9 \mathrm{~s}$. The lower right panel is a blow-up of the acf near the lag of a two-way light time (indicated by the vertical line). 
white phase noise due to finite signal-to-noise ratio; see Section 4.7). In a controlled test at DSS 25 (antenna stationary, FTS common to the transmit and received chains and thus cancelled in this zero two-way light time test) the sum of the noises from the exciter, transmitter, downconversion electronics, and receiver was measured (Figure 5). The power spectrum of those test data is shown in [1]; the corresponding Allan deviation at $\tau=1000 \mathrm{~s}$ is $2 \times 10^{-16}$.

\subsection{Spacecraft transponder noise}

Transponders accept an input carrier signal and produce an output signal at a different frequency. The process is phase-coherent; that is, for every $N$ integer cycles of the input there are $M$ integer cycles of the output (with $M / N$ being the transponding ratio). When this condition is achieved, the transponder is operating normally and produces an output that is "locked" to the input signal. The Cassini spacecraft has two transponders". The standard flight transponder ("KEX") accepts the X-band uplink and produces two phase-coherent outputs, one at $f_{\mathrm{X}} \cdot 880 / 749(=\mathrm{X}$-band downlink frequency) and another at $f_{\mathrm{X}} \cdot 3344 / 749$ (= Ka1 downlink frequency), where $f_{\mathrm{X}}$ is the frequency of the X-band uplink signal observed at the spacecraft. These signals are amplified, routed to the spacecraft high-gain antenna, and transmitted to the earth. Another flight unit, the Ka-band Translator ("KaT"), accepts a Ka-band uplink signal and produces a phase coherent signal with frequency $f_{\mathrm{k}} \cdot 14 / 15$ (= Ka2 downlink frequency), where $f_{\mathrm{k}}$ is the Ka-band signal frequency observed at the spacecraft.

Pre-launch test data of transponders similar in design to Cassini's KEX showed negligible (i.e., $<10^{-15}$ ) frequency noise. Prelaunch tests of the KaT similarly showed negligible frequency noise $\left(\simeq 10^{-16}\right.$ at $\left.\tau=1000 \mathrm{~s}\right)$, provided the received Ka-band uplink signal was relatively strong (greater than about $-127 \mathrm{dBm}$, see $[1,19,78,22])$ at the input to the KaT.

Appropriate linear combinations of the frequency time series of the three downlinks can be used to estimate and remove (at the Fourier frequencies of interest) downlink and round-trip plasma noise [68, 65, 69, 70] in GW observations. For example, the downlink plasma noise time series can be determined by forming $f_{\mathrm{X}}-(880 / 3344) f_{\mathrm{Ka} 1}$, which is independent of FTS noise, antenna mechanical noise, spacecraft buffeting, and GWs (since these are all nondispersive.) These plasma corrections were also used with good success by Bertotti, Iess, and Tortora [29] in a precision test of relativistic gravity involving Cassini tracking very close to the sun.

\subsection{Thermal noise in the ground and spacecraft receivers}

Finite signal-to-noise ratios in the up- and downlinks cause white phase noise. For observations to date, the signal-to-noise ratio (SNR) of the downlink dominates. The one-sided spectral density of phase fluctuations due to finite SNR, $S_{\phi}$, is $\simeq 1 /\left(\mathrm{SNR}\right.$ in $1 \mathrm{~Hz}$ band) $\operatorname{rad}^{2} \mathrm{~Hz}^{-1}$. The associated Allan deviation [23] is $\simeq \sqrt{3 B S_{\phi}} /\left(2 \pi \nu_{0} \tau\right)$, where $B$ is the bandwidth of the detection system and assuming $2 \pi B \tau \gg 1$. For Cassini gravitational wave observations, $B$ is typically $\simeq 1 \mathrm{~Hz}$ and the typical X- or Ka-band SNR in a $1 \mathrm{~Hz}$ bandwidth can be $45 \mathrm{~dB}$ or more. At $\tau=1000 \mathrm{~s}$ finite link SNR contributes negligibly to the overall noise budget in current generation Doppler experiments (see Table 2).

\subsection{Spacecraft unmodeled motion}

Unmodeled motion of the spacecraft enters directly into the two-way Doppler time series (see Figure 7). The lack of a time-domain signature makes it difficult to isolate spacecraft motion using the Doppler data only. Such unmodeled motion can arise in principle from a variety of

\footnotetext{
7 In principle, Cassini has one transponder and one translator. The distinction is that a transponder performs functions in addition to phase-coherent generation of the downlink signal from the uplink signal.
} 
causes: fluctuations in the solar wind hitting the spacecraft, fluctuations in solar radiation pressure, physical articulation of spacecraft parts, leaking thrusters, sloshing of fuel in the spacecraft's tanks, etc. Solar wind and solar radiation pressure fluctuations are computed to be far too small to affect observations at current levels of sensitivity [100]. Articulation of instruments is restricted during quiet spacecraft periods where good Doppler sensitivity is required. Leaking thrusters and fuel sloshing are also thought to be small effects at current generation Doppler sensitivity [100].

In one case the as-flown spacecraft motion noise was independently determined. Using telemetry from Cassini's reaction wheel assembly, Won, Hanover, Belenky, and Lee [129] inferred the time series of antenna phase center motion projected onto the earth-spacecraft line (i.e., the sensitive axis for the Doppler system). This test was done when Cassini was in semi-quiet cruise (thrusters off but with physical articulation of elements of one science instrument, the Cassini Plasma Spectrometer, at $\simeq 0.0025 \mathrm{~Hz}$ ) for 40 hours during 2001 DOY 152-153. The resulting Allan deviation for unmodeled spacecraft motion at $\tau=1000 \mathrm{~s}$ was computed to be $2.3 \times 10^{-16}$. Figure 13 shows the spectrum of velocity noise observed in that test. Unmodeled motion of the spacecraft - at least the Cassini spacecraft - is thus negligible compared with other noises at the sensitivity of current-generation Doppler experiments (see Figure 10 and Table 2).

\subsection{Numerical noise in orbit removal}

Removal of the systematic variation in the Doppler time series due to the known motion of the earth and spacecraft can be done using the JPL/NASA Orbit Determination Program (ODP; [85]) or its successor the Mission-analysis, Operations, and Navigation Tool Environment (MONTE). Subtraction of the computed systematic Doppler frequency from the observed time series then gives residuals which can be searched for gravitational waves. The ODP computes Doppler by differencing the computed range to the spacecraft at two separated times and dividing by the time difference. Because of finite computer word length neither range nor time is expressed with perfect accuracy. These, coupled with finite-accuracy computer arithmetic, give rise to numerical noise which propagates to the residuals. Because of the granularity in time and distance the magnitude of numerical noise depends on time past 12:00 UT January 1, 2000 (the ODP measures time from J2000), distance and relative radial velocity of the spacecraft [133] and is small compared with other noises for the early 2000s Cassini gravitational-wave observations (thus not included in Table 2). However, it is clear in Cassini Doppler data taken later in the Saturn tour [133, 67] and likely relevant for future, e.g., Juno and BepiColombo, observations.

\subsection{Aggregate spectrum}

Figure 14 shows the two-sided power spectrum of two-way fractional Doppler frequency, $S_{y 2}(f)$, computed from data taken at DSS 25 during the 2001-2002 solar opposition (from [19]). It is derived after using the multi-link plasma corrections and the AMC tropospheric calibrations. The intrinsic frequency resolution of the spectrum is about $3 \times 10^{-7} \mathrm{~Hz}$. The spectrum in Figure 14 is smoothed to a resolution bandwidth of $3 \times 10^{-6} \mathrm{~Hz}$ to reduce estimation error. Approximate $95 \%$ confidence limits for the logarithm of an individual smoothed spectral estimate are indicated [73, 89].

The low-frequency part of the spectrum in Figure 14 consists of a continuum plus spectral lines between $\simeq 10^{-5}-10^{-4} \mathrm{~Hz}$. The lines in the unsmoothed spectrum are near the resolution limit of the 40 day observation; their apparent width in Figure 14 is due to the spectral smoothing used to reduced estimation error. The lowest frequency line is near one cycle/day; the other lines are near harmonics of one cycle/day. Because of the multi-link plasma correction, all random processes contributing to this spectrum are non-dispersive. At frequencies greater than about $1 / T_{2}$ there is clear, approximately cosinusoidal, modulation. This is characteristic of positive correlation in 


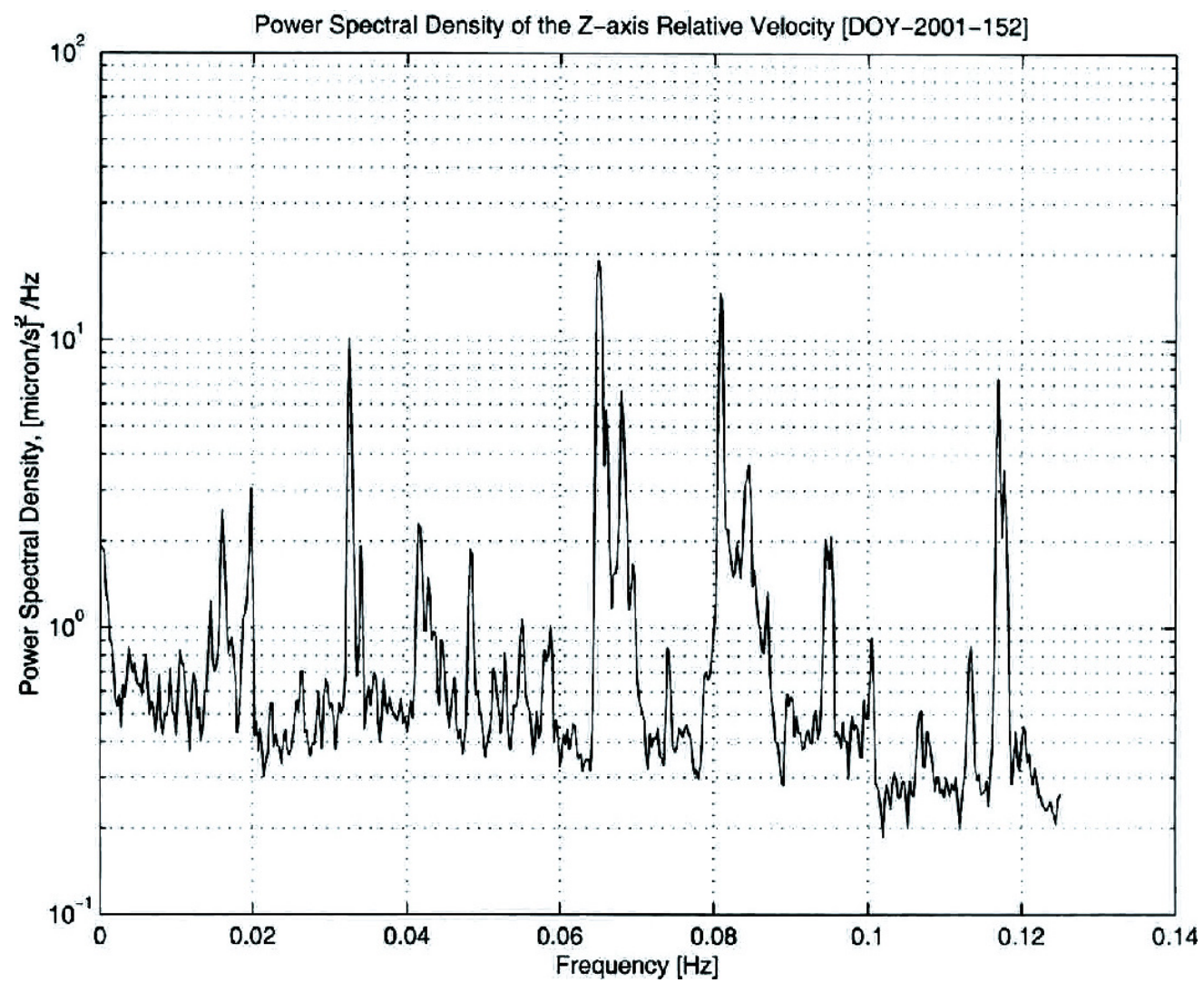

Figure 13: Spectrum of Cassini radial velocity fluctuations, observed in a 40-hour cruise test during 2001 DOY 152-153 [129], reproduced here with permission. The Allan deviation associated with this spacecraft buffeting noise $[52,46]$ is $2.3 \times 10^{-16}$ at an integration time of $1000 \mathrm{~s}$.

the time series at lag $\tau=T_{2}$, i.e., either antenna mechanical noise or residual tropospheric noise. The level is too large to be dominated by residual tropospheric scintillation, however, and so is interpreted as mechanical noise. Many minima of the mechanical noise transfer function - at odd multiples of $1 /\left(2 T_{2}\right)$ - are easily visible in Figure 14 . The spectrum appears to continue to be dominated by mechanical noise up to $\simeq 0.01 \mathrm{~Hz}$, with the signature of the transfer function being, however, difficult to see on this log plot (and also blurred at high frequencies since $T_{2}$ changed with time by about $3 \%$ over the course of the 40 day observation.)

\subsection{Summary of noise levels and transfer functions}

To summarize the noise model: The principal noises are frequency and timing noise (FTS), plasma scintillation (solar wind and ionosphere), spacecraft electronics, unmodeled spacecraft motion, unmodeled ground antenna motion, tropospheric scintillation, ground electronics noise, thermal noise in the receiver, and systematic effects. The magnitudes of these noises in Cassini-era (20012008) observations are given in Table 2. Before any corrections, these noises enter the two-way Doppler as

$$
\begin{aligned}
y_{2}^{\text {noise }}(t)= & y^{\mathrm{FTS}}(t) *\left[\delta(t)-\delta\left(t-T_{2}\right)\right]+y^{\mathrm{sw}}(t) *\left[\delta(t)+\delta\left(t-T_{2}+2 x / c\right)\right]+ \\
& y^{\text {ion }} *\left[\delta(t)+\delta\left(t-T_{2}\right)\right]+y^{\mathrm{s} / \mathrm{c} \mathrm{elect}}(t) * \delta\left(t-T_{2} / 2\right)+y^{\mathrm{s} / \mathrm{c} \text { motion }} * \delta\left(t-T_{2} / 2\right)+
\end{aligned}
$$




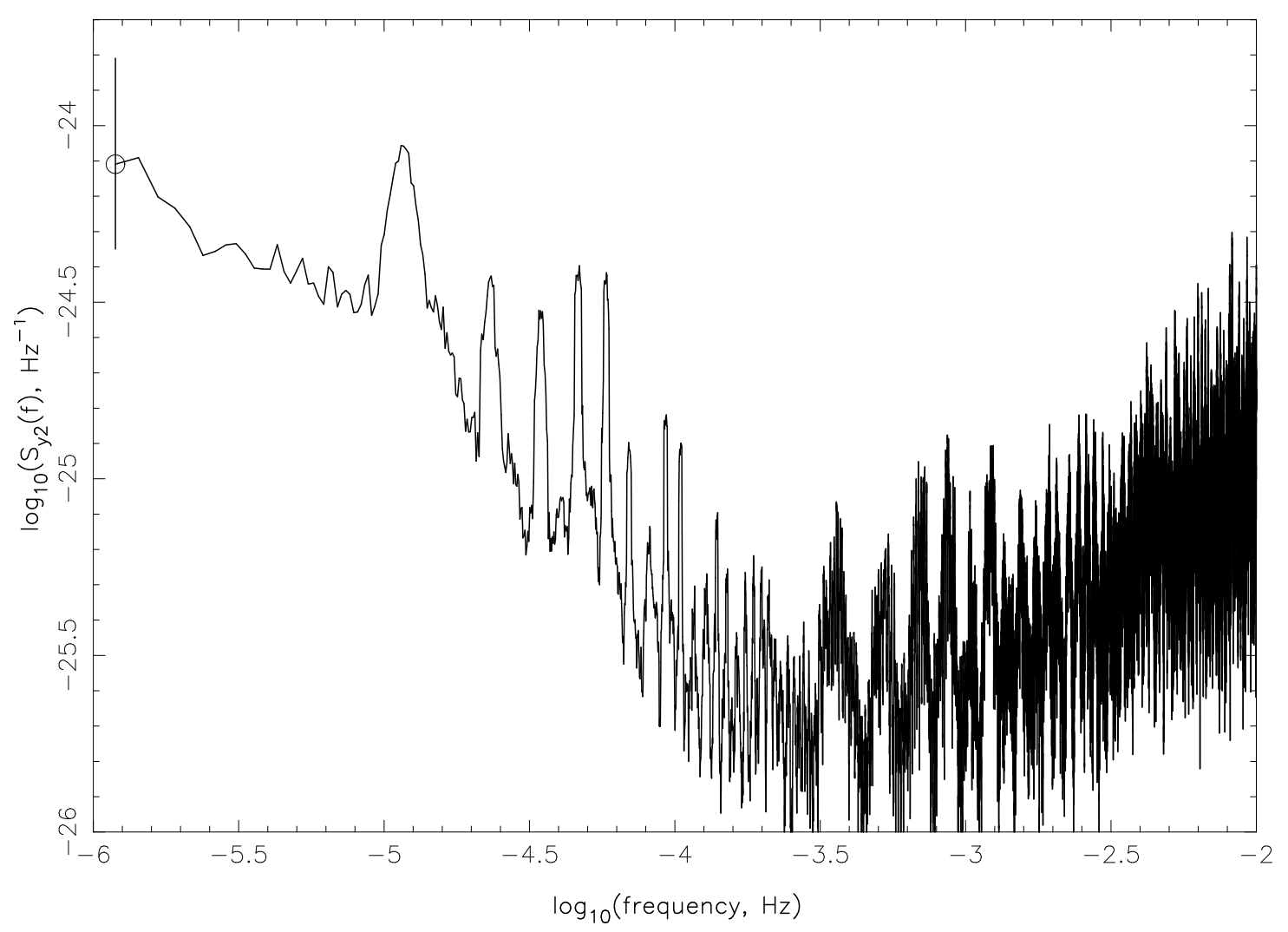

Figure 14: Power spectrum of the two-way fractional frequency fluctuations (calibrated for plasma and troposphere) for 2001-2002 Cassini solar opposition observations (adapted from [19]). The spectrum has been smoothed from the intrinsic resolution of the observations to a bandwidth of $\simeq 3 \mu \mathrm{Hz}$ to reduce estimation error. Representative $95 \%$ confidence limits are indicated.

$$
\begin{aligned}
& y^{\text {ant }}(t) *\left[\delta(t)+\delta\left(t-T_{2}\right)\right]+y^{\text {tropo }}(t) *\left[\delta(t)+\delta\left(t-T_{2}\right)\right]+y^{\text {ground elect }}(t)+ \\
& y^{\text {rcvr }}+y^{\text {systematic }}(t)
\end{aligned}
$$

where $x$ is the effective distance of the solar wind perturbation from the earth. After multi-link plasma calibration, phase scintillation due to charged particles is effectively removed. Water-vaporradiometer-based tropospheric calibration removes $\simeq 90 \%$ of the low-frequency fluctuations due to the neutral atmosphere, so that the calibrated time series $y_{2}$ is approximately

$$
\begin{aligned}
y_{2}(t) \simeq & y_{2}^{\mathrm{gw}}+y^{\mathrm{FTS}}(t) *\left[\delta(t)-\delta\left(t-T_{2}\right)\right]+y^{\mathrm{s} / \mathrm{c} \mathrm{elect}}(t) * \delta\left(t-T_{2} / 2\right)+ \\
& y^{\mathrm{s} / \mathrm{c} \text { motion }} * \delta\left(t-T_{2} / 2\right)+y^{\text {ant }}(t) *\left[\delta(t)+\delta\left(t-T_{2}\right)\right]+y^{\text {tropo }}(t) / 10 *\left[\delta(t)+\delta\left(t-T_{2}\right)\right]+ \\
& y^{\text {ground elect }}(t)+y^{\mathrm{rcvr}}+y^{\text {systematic }}(t) \\
= & y_{2}^{\mathrm{gw}}+y_{2}^{\text {other }}(t)
\end{aligned}
$$

where $y_{2}^{\text {other }}(t)$ is all the non-GW (noise plus systematics) contributions to the two-way Doppler variability.

Table 2 summarizes the noise model and the associated Allan deviation at $\tau=1000 \mathrm{~s}$ for the principal noises (models of the spectra of the individual noises are given in [111]). Figure 5 shows, highly schematically, the signal flow. This sketch, the GW transfer function (see Eq. (1)), and the 
noise transfer functions (see, e.g., Figure 7) are used in discussions of sensitivity, signal processing, and for qualifying/disqualifying candidate GW events.

Table 2: Noise sources and contributions (expressed as Allan deviation [23] at $1000 \mathrm{~s}$ integration) in Cassini-era precision Doppler tracking GW observations. This table is adapted from [1, 22]. SEP: sunearth-probe (solar elongation) angle; SNR: signal-to-noise ratio of the downlink. Models of the spectra of the individual noises are given in [111].

\begin{tabular}{|c|c|c|}
\hline Noise source & $\sigma_{y}[1000 \mathrm{~s}]$ & Comment \\
\hline Frequency standard & $\simeq 8 \times 10^{-16}$ & fundamental noise source $[1,22]$ \\
\hline Antenna mechanical & $\simeq 2 \times 10^{-15}$ & $\begin{array}{l}\text { DSS } 25 \text { (34-m antenna) under fa- } \\
\text { vorable conditions [19] }\end{array}$ \\
\hline Ground electronics & $\simeq 2 \times 10^{-16}$ & $\begin{array}{l}\text { measured in controlled test, } \\
\text { DSS } 25[1]\end{array}$ \\
\hline Plasma phase scintillation & $\begin{array}{l}<10^{-15}, \text { for Ka-band and } \\
\text { SEP }>150^{\circ}\end{array}$ & $\begin{array}{l}\sigma_{y} \text { depends on SEP; dispersive } \\
\text { scales as square of radio wave- } \\
\text { length; see Figure } 10\end{array}$ \\
\hline Stochastic spacecraft motion & $\simeq 2 \times 10^{-16}$ & $\begin{array}{l}\text { Cassini in quiet cruise }[129,130] \\
\text { see Figure } 13\end{array}$ \\
\hline Receiver thermal noise & few $\times 10^{-16}$ & $\begin{array}{l}\text { depends on link SNR and detec- } \\
\text { tor bandwidth [23] }\end{array}$ \\
\hline Spacecraft transponder noise & $\simeq 10^{-16}$ & $\begin{array}{l}\text { preflight tests of Cassini Ka- } \\
\text { band translator }[1,22]\end{array}$ \\
\hline $\begin{array}{l}\text { Tropospheric scintillation } \\
\text { (raw) }\end{array}$ & $<3 \times 10^{-15}$ to $\simeq 30 \times 10^{-15}$ & variable; nondispersive $[20,75]$ \\
\hline $\begin{array}{l}\text { Tropospheric scintillation } \\
\text { (corrected) }\end{array}$ & $<1.5 \times 10^{-15}$ to $\simeq 3 \times 10^{-15}$ & $\begin{array}{l}\text { under favorable conditions } \\
\text { median conditions in connected } \\
\text { element interferometry tests }\end{array}$ \\
\hline
\end{tabular}




\section{Signal Processing}

This section discusses signal processing approaches, both formal and heuristic, which have been used to analyze Doppler observations. These include noise spectrum estimation, analysis methods to search for periodic, burst, and stochastic signals, and ideas for qualifying/disqualifying candidate signals based on signal and noise transfer functions.

\subsection{Noise spectrum estimation}

Spacecraft Doppler tracking shares attributes with all other real observations: The noise is nonstationary, there are low-level systematic effects, and there are data gaps. The noise can usually be regarded as effectively statistically stationary for at most the interval of a tracking pass ( $\simeq 8$ hours). The non-stationarity of (what may be fundamentally Gaussian but with time-variable variance) noise is a complication. For example in matched filtering for bursts, discussed below, the noise spectrum has to be estimated locally [63] for use in deriving locally-valid matching functions from the assumed GW waveforms [61].

Noise characterization has historically been done via standard spectral and acf analysis techniques [73]. Spectra of various data sets have been presented in $[4,18,32,9,19]$. The data have typically been analyzed with varying time-frequency resolution to assess the fidelity of the spectral estimates and to provide local (in Fourier frequency) estimates of the underlying noise spectral density for sinusoidal and chirp signal searches (below). Running estimates of the variance and third central moments have been used as guides for identifying intervals of stationarity in pilot studies. Bispectra ${ }^{8}$ were computed for early data sets looking for non-linear, non-Gaussian effects. Bispectral analysis seemed to have limited utility, however; the Doppler noise is close to Gaussian and the slow convergence of higher statistical moments makes the bispectrum hard to estimate accurately over the length of a stationary data interval.

\subsection{Sinusoidal and quasiperiodic waves}

Some candidate sources radiate periodic or quasiperiodic waves. From an observational viewpoint a signal is effectively a sinusoid if the change in wave frequency over the duration of the observation $T$ is significantly less than a resolution bandwidth $1 / T$. Since in a search experiment the signal phase is not known, spectral analysis $[73,107,89]$ is appropriate. In the absence of a signal, the real and imaginary parts of the time series' Fourier transform are Gaussian and uncorrelated, so the Fourier power is exponentially distributed [88]. In the presence of a signal, the Fourier power is "Rice-squared" distributed [97]. Formal tests for statistical significance [55, 107, 4, 18, 7, 5, 89] involve comparing the power in a candidate line with an estimate of the level of the local noisespectrum continuum. Since the frequency of the signal is also not known a priori, a range of frequencies must be searched. The spectral power is approximately independent between Fourier bins, so the joint probability density function of the power in $n$ Fourier bins is the product of the individual bin pdfs. This can be used to set statistical confidence limits for the sensitivity of a search experiment over multiple candidate signal frequencies [18].

A signal is effectively a linear chirp if the change in frequency over the observation interval is $>1 / T$ but the curvature of the signal's trajectory in a frequency-time plot is negligible over the observing interval. In this case, signal power is smeared in (frequency, time) and simple spectral analysis is inappropriate. In a chirp-wave analysis the Doppler data are first passed through a software preprocessor which tunes the signal to compensate for the linear chirping. With the

\footnotetext{
8 The bispectrum is the Fourier transform of the third-order lagged product $\left\langle x(t) x\left(t+\tau_{1}\right) x\left(t+\tau_{2}\right)\right\rangle$; it gives the contribution to the third moment from the product of three Fourier components having frequencies which add to zero. It has been used in many fields, notably geophysics, to study weak nonlinearities (see, e.g., [56, 80]).
} 
correct tuning function, the chirp is converted to a sinusoid in the output. Spectral analysis is then used to search for statistically significant lines. The tuning function $\exp \left(i \pi \beta t^{2}\right)$ is complex. The parameter $\beta$, an estimate of $d f / d t$, is unknown and must be varied. (In an idealized situation, this procedure resolves the signal into three lines, with frequency separation depending on $\beta$ and $T_{2}$ [5]. The observability of all three lines is unlikely in real observations, however.) The situation is different from the sinusoidal case in that an arrow of time has been introduced by the software dechirping; the positive and negative frequency components of the dechirped spectrum contain different information. In principle, an ensemble of chirping signals, each too weak to be detected individually, could be identified by noting differences in the statistics of the positive and negative frequency components of the dechirped spectrum.

Waveforms which are more complicated than linear chirps arise, e.g., from binary systems near coalescence. To do proper matched filtering [61] the waveform and the source location on the sky are needed. If one assumes the time evolution of the phase, the time series can be resampled at unequal times $[102,5]$ so that (in terms of the resampled phase variable) the signal is periodic. This suboptimum technique can be used in pilot analyses to pre-qualify candidates for exact matched filtering. Nonsinusoidal periodic waves, generated, e.g., by non-circular binary systems, can have rich Fourier content [127]. Searches for these waveforms have included folding the data with assumed periods [18].

\subsection{Bursts}

Bursts are time-localized signals in the data set. Matched filtering with assumed waveforms involves varying several parameters, including $\cos \theta$, in the three-pulse response. Burst searches are helped by the very diagnostic three-pulse response (integral of signal response must be zero; location and amplitude ratios of the "pulses" must be consistent with $\theta$.) Matched filter outputs have a "signal part" (integral of the matching function with the signal) and a "noise part" (integral of the matching function with the noise). The variance of the matched-filter's noise-only output changes if the noise is non-stationary. If not accounted for this can result in distorted pdfs of matched filter outputs and (superficially significant) tails of the distribution of matched filter outputs, even in the absence of a signal. To allow for this [63] the data can be divided into intervals over which the noise appears stationary. A model of the noise spectrum over each interval is used, along with the assumed signal waveform, to compute the matching function. This matching function is then used for that interval only. Simulation of the matched filter against synthetic noise having the same spectrum and data gap structure of the interval being analyzed is used to estimate the variance of the noise-only matched filter output. Then the actual matched filter outputs can be normalized by the estimated noise-only variance to express outputs in terms of SNRs. This allows the outputs of the matched filter to be compared consistently across a data set where the noise statistics are changing. Multi-spacecraft coincidences can be used to reduce further false-alarms [28, 112, 63, 13].

Related to burst processing are "template independent" methods for identifying data intervals for more detailed study. Wavelet transforms of the data on a pass-by-pass basis have been sometimes useful in finding time-localized intervals formally contributing anomalously large variance. These are then typically checked to see if there are corresponding features within $\pm T_{2}$. Examination of the time series reconstructed from some small fraction $(\simeq 10 \%)$ of the largest amplitude wavelets (or systematically from the wavelets in selected subbands only) have also been useful [11].

\subsection{Stochastic background}

Stochastic background limits can be derived from the smoothed Doppler frequency power spectrum $[52,26]$. If the background is assumed isotropic, spectra of two-way Doppler can be converted to spectra of strain by dividing by the sky- and polarization-averaged GW response func- 
tion $[52,54,19]$. (If a background is not isotropic, then the spectral response function is the square of the Fourier transform of the three-pulse response evaluated for the relevant $\mu$ and averaged over polarization states.) From $S_{h}$ the strength of the background can be expressed relative to closure density or as a characteristic strain amplitude [108, 93, 72] (see Section 6.5).

\subsection{Classification of data intervals based on transfer functions}

Although the signal waveforms are not known a priori, there is a good understanding of transfer functions of the GW signal and the principal noises to the Doppler. Partitioning the data into "known-noise-like" or "other" intervals based on the noise transfer functions can be useful. Examples of discrete-event noise classification based on transfer function were shown in Section 4; statistical classification of data intervals based on the local acf is also possible (see, e.g., $[9,19])$. The local spectrum or correlation function has also been used to assess the relative importance of different noises and their stationarity from, e.g., the degree of correlation at $\tau=T_{2}$.

\subsection{Frequency-time representations}

It is often useful to think of signal representations in a frequency-time phase space, shown schematically in Figure 15. There are many ways to tile frequency-time, e.g., Fourier transforms, wavelets, chirplets, Gabor-transforms (and variants, depending on the temporal windowing used); there is a correspondingly large literature. Depending on the situation each tiling can have special merit (e.g., if additional information suggests a specific candidate signal is likely to project preferentially onto a small fraction of a particular mathematical basis while the noise does not).

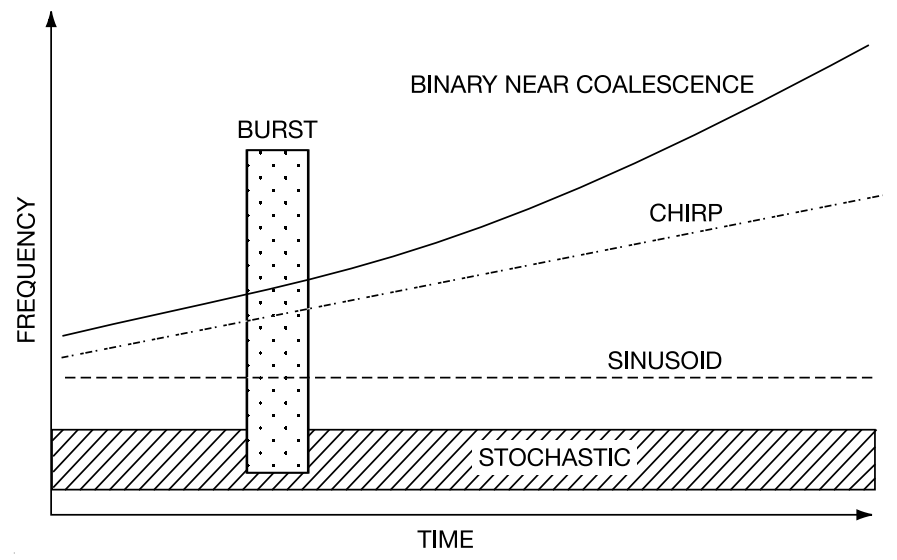

Figure 15: Schematic diagram of signals in a frequency-time space. Sinusoids are "on" for all time and have horizontal tracks, linear chirps are straight lines with non-zero slope, bursts are time-localized, etc. These localizations in frequency-time suggest different detection approaches for different classes of signal. This space can be tiled in many ways. Particular tilings can have special merit for particular waveforms, e.g., if a candidate signal projects preferentially onto a small fraction of a particular mathematical basis while the noise does not.

As an example, Figure 16 shows normalized Fourier power as a function of frequency-time for the Cassini two-way Ka-band track on 2001 DOY 350 (time series shown in Figure 8). This plot was constructed by taking the unwindowed power spectrum of sequential $102.4 \mathrm{~s}$ data segments (in this case $75 \%$ overlapped in time). The heavy white line indicates the two-way light time at the beginning of the data set. The normalized power - power at a given (frequency, time) point divided by the estimated local continuum power near that point - is plotted. This is a nondimensional 
measure of the contrast (and potential statistical significance) of the Fourier power at that point relative to a local background. The color code runs from black (low values) through green to red (very high values). Points with estimated contrast ratio $>10$ are marked with white circles. If two high-contrast features are at the same frequency and separated by a two-way light time, they are connected with a thin white line. The FTS glitch of Figure 8 is clearly evident in both the time series and in $T_{2}$-separated bands of high-contrast Fourier power in the lower plot. Additional features not evident in the time series but paired at $T_{2}$ are detected near the beginning of the data set and at low-frequency. See also Figures 17 and 18.

\subsection{Qualifying/disqualifying candidates}

Qualifying or disqualifying candidate signals is based both on spectra of noise processes and, usually more crucially, on signal and noise transfer functions. In some cases it is immediately obvious, using the noise transfer function and a single time series, that a stretch of data is noise dominated (large antenna mechanical events for example). In other cases, multiple time series (e.g., the multiple $\mathrm{X}$ - and Ka-band signals available with the Cassini observations; see Figure 5) can be used to qualify candidates.

As an example, candidate periodic and quasiperiodic signals have been disqualified in various data sets (discussed below) based on one or more of these considerations:

- Is there a radio wave amplitude variation associated with a Doppler variation (for GWs, there should not be)?

- Is there something special about the data taking or the data records where the candidate is observed?

- Is the back-end instrumentation distinguished in some way (e.g., common receiver or receiver rack)?

- Is the candidate observed only at a specific tracking-station? (Is the candidate observed at only one kind of station, e.g., only at the DSN beam-waveguide antennas and not at the DSN "high-efficiency" antennas?)

- Is the signal a plausible alias of a man-made frequency?

- Could a narrow-band feature be due to a mechanical resonance of the antenna?

- Does it appear in only part(s) of the tracking record?

- Is its frequency modulation consistent with the source being at a fixed point on the sky, given the known earth-spacecraft geometry variation over the observation?

- Is the candidate observed in more than one radio band (e.g., X- and Ka-band) simultaneously? Is it demonstrably non-dispersive? Are the individual-band SNRs consistent with a common $\Delta v / c ?$

- Is the candidate consistent when observed with different resolutions in time and Fourier frequency?

- Could this candidate have been introduced into the time series by a faulty calibration?

Each of the above has been used to assess reality or unreality of candidate periodic and quasiperiodic waves in different data sets. Some published examples include the following ones:

Anderson et al. [5] observed a chirp that persisted over 10 days. The data were reanalyzed in subsets based on inclusion or exclusion of specific stations, specific transmitter/receiver pairs, 


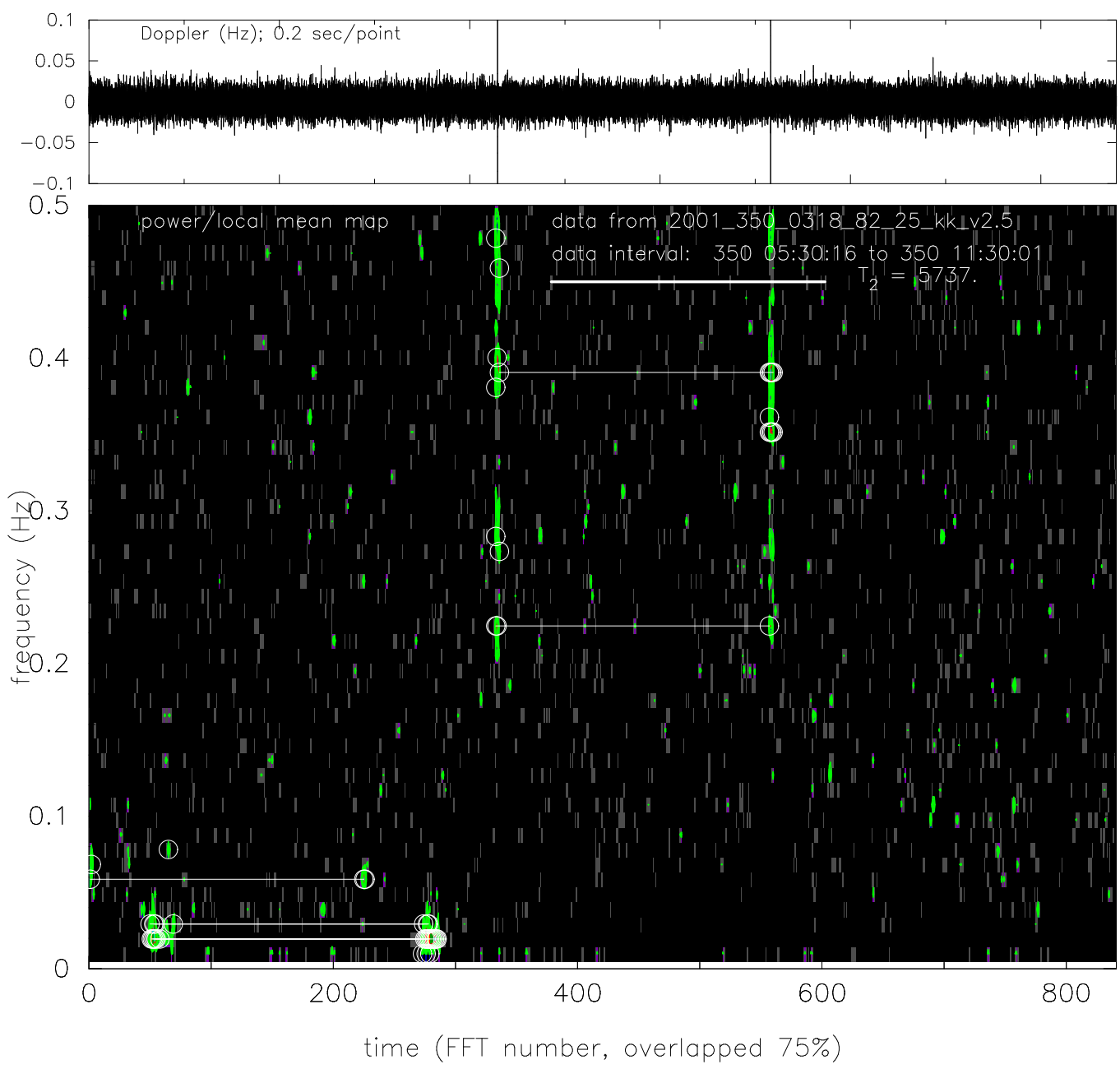

Figure 16: Frequency-time representation of Cassini two-way Ka-band data on 2001 DOY 350. Upper panel: time series of two-way Doppler data with $\simeq 1 \mathrm{~s}$ time constant, sampled at $0.2 \mathrm{~s} / \mathrm{sample}$. At this resolution the visual impression of the plot is set by relatively high frequency noise. Lower right panel: low frequency resolution power spectrum of the full data set shown in upper panel. Lower left panel: normalized dynamic spectrum of the data in the upper panel. This was constructed by forming sequential spectra of short $(\simeq 102 \mathrm{~s})$ unwindowed segments of the data. Each data segment is $75 \%$ overlapped with its neighbors. The heavy white line indicates the two-way light time at the beginning of the data set. The plotted quantity is power at a given point in (frequency-time) divided by a local estimate of the average power at that (frequency, time) point, a nondimensional measure of the contrast of the Fourier power at that point relative to an estimated background. The color code runs from black (low values) through green (higher values) to red (very high values). Points with this estimated contrast ratio $>10$ are marked with white circles. If two high-contrast features are at the same frequency and separated by a two-way light time, they are connected with a thin white line. The FTS glitch shown also in Figure 8 is clearly evident in both the time series and in $T_{2}$-separated bands of high-contrast Fourier power in the lower plot. Additional features paired at $T_{2}$ in the earlier, lower-frequency part of the data are also detected in the normalized dynamic spectrum. 
and temporal partitions of the data set. The chirp was ultimately disqualified as non-astronomical because it was only observed in the subset of the data involving a particular transmitter/receiver pair.

In another Pioneer spacecraft observation [18] the statistical significance of candidate spectral lines was assessed by scrambling the data within the data gaps and reanalyzing. This confirmed analytical work on the false alarm probability and the (lack of) statistical significance of the strongest candidate periodic signals.

In the three-spacecraft coincidence experiment involving the Galileo, Mars Observer, and Ulysses spacecraft [13] matched filtering for signals from particular directions that were sinusoidal (except for modulation due to earth-spacecraft motion modulation over the course of the $\sim 20$-day track) gave undistinguished peak SNRs in the Mars Observer and Galileo data sets, but a formally significant SNR in the Ulysses data set. From the modulation over the period of the observations, the inferred direction of arrival was $\alpha \simeq 0^{\circ}, \delta \simeq+69^{\circ}$. Since the position of the candidate source was thus "known", gravitational-wave polarization states [127, 18, 16] were explored looking for states which could simultaneously couple well to Ulysses but poorly (so as to push a real astronomical signal into the noise) to Mars Observer and Galileo. There was no polarization state which could produce this simultaneously in the three data sets, so this candidate was excluded as a false alarm.

The consistency of multiple, simultaneous data sets can also be used to qualify or disqualify non-periodic waveform candidates. This is complicated by the fact that different data sets have different noise levels and thus different sensitivities to GWs. Figure 17 shows a normalized dynamic spectrum for Cassini two-way Ka-band data on 2003 DOY 008. The strong feature observed in the average spectrum (right panel) comes from a short time interval in the track at about 08:50 UT. Figure 18 shows the dynamic spectrum of two-way X-band for the same track, with no highcontrast feature near (08:50 UT, $0.22 \mathrm{~Hz})$. Subsequent analysis of band pass filtered time series for the X-and Ka-band data showed that the event seen in the Ka-band data, if produced by a real earth-spacecraft velocity, should have also been observable above the noise in the X-band and was not. Such events were observed once per day in the 2002-2003 Cassini observing campaign (only - and with varying strength) and are apparently a systematic effect specific to the two-way Ka-band system (perhaps associated with the independence of the Ka-band transmit and receive horns; see Section 4.4 and Figure 5).

Qualifying/disqualifying candidate burst waves is slightly different because, by hypothesis, the signal is only "on" for a finite time and some of the above tests do not apply. However a true GW burst must be nondispersive and show the correct three-pulse signature in the time series. Here the three-pulse response [52] is very powerful: Whatever the GW waveforms, the signal must show the three-pulse response with correct amplitudes and spacings for a GW from a specific direction relative to the earth-spacecraft line (see Figure 1).

\subsection{Other comments}

Another analysis scheme which is attractive in principle but does not seem useful in currentsensitivity Doppler data analysis is the Karhunen-Loéve expansion [61, 43]. This is a signalindependent approach where the data themselves are used to construct a mathematical basis to express the data. Such representations may be useful for template-free analysis of time series dominated by a signal of unknown waveform. However, experiments with this analysis procedure on simulated noise-dominated Doppler data sets were disappointing; the modes discovered in the Karhunen-Loéve simulations were always the noise modes.

Editing flags developed, e.g., from spacecraft telemetry or from DSN tracking logs have not historically been useful ${ }^{9}$ as veto signals (the internal monitoring capabilities of the spacecraft and

\footnotetext{
9 An exception was with Mars Observer [63, 13] where spacecraft engineering telemetry was crucial for correcting
} 


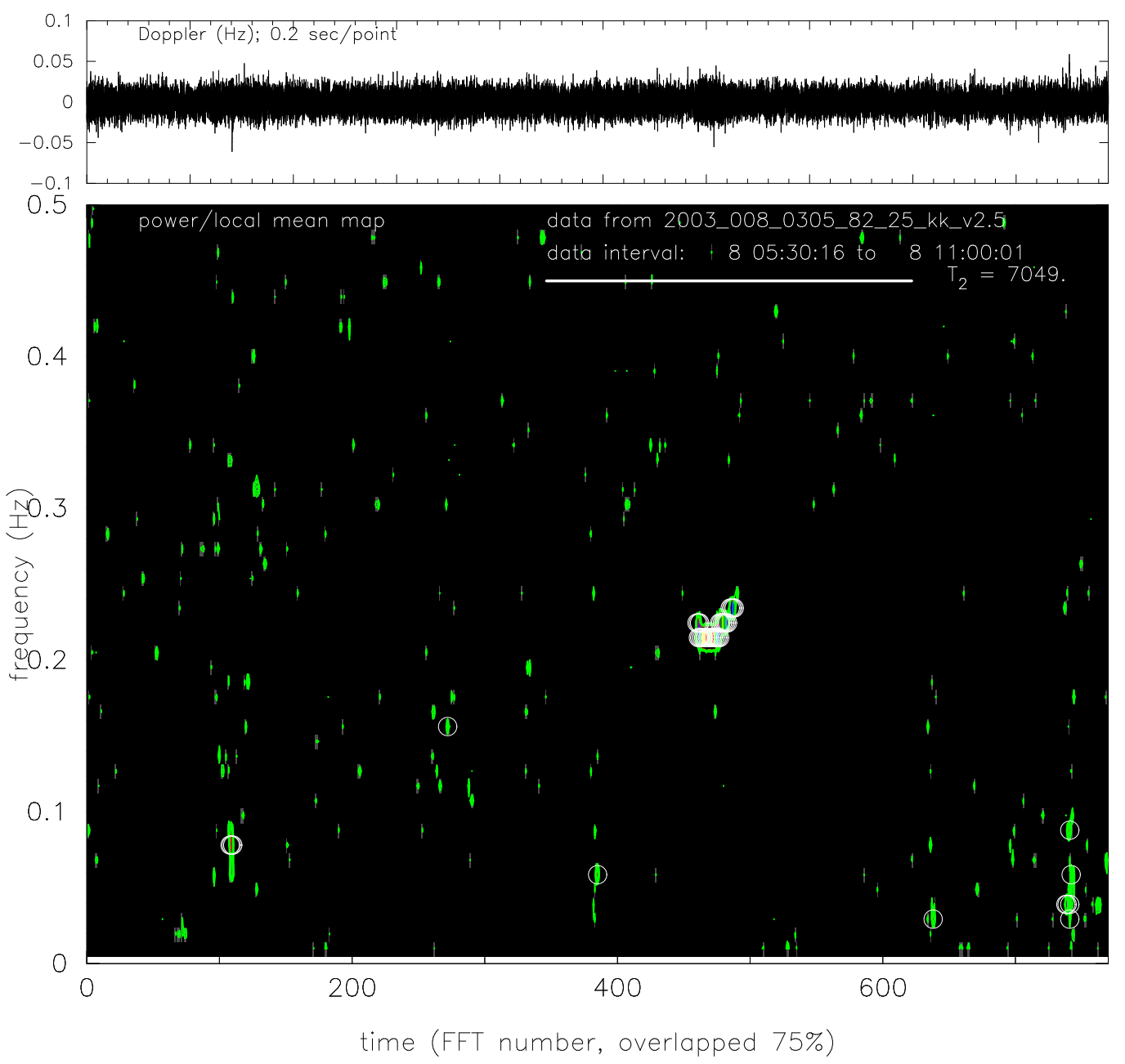

Figure 17: As for Figure 16, but for the Cassini two-way Ka-band track on 2003 DOY 008. The strong features in the dynamic spectrum at about $(08: 50,0.22 \mathrm{~Hz}$ ) have peak local contrast $>100$ (and are even marginally visible in the time series in the upper panel).

ground stations were, of course, not intended for this purpose). The Doppler itself is much more sensitive than the system monitors and also - being spatially distributed by $c T_{2} / 2$ - has noisesignatures which often allow easier identification of specific disturbances affecting the time series (see, e.g., Section 4).

Even though each class of tracking antenna has a common design, there are low-level stationspecific systematic differences. Getting data with different stations helps at least to identify these systematics (see, e.g., [5]). Also data taken at low elevation angle $\left(<20^{\circ}\right)$ with any antenna are statistically of poorer quality.

Finally, at current levels of sensitivity Doppler tracking observations are clearly search experiments. We are looking for signals with poorly-constrained waveforms which are "surprisingly" strong (thus expected to be rare). To maximize the chance that an unexpected real event will not be dismissed as due to a known noise process (or overlooked altogether), it is obviously useful

the Doppler for the (slow) spacecraft spin. 


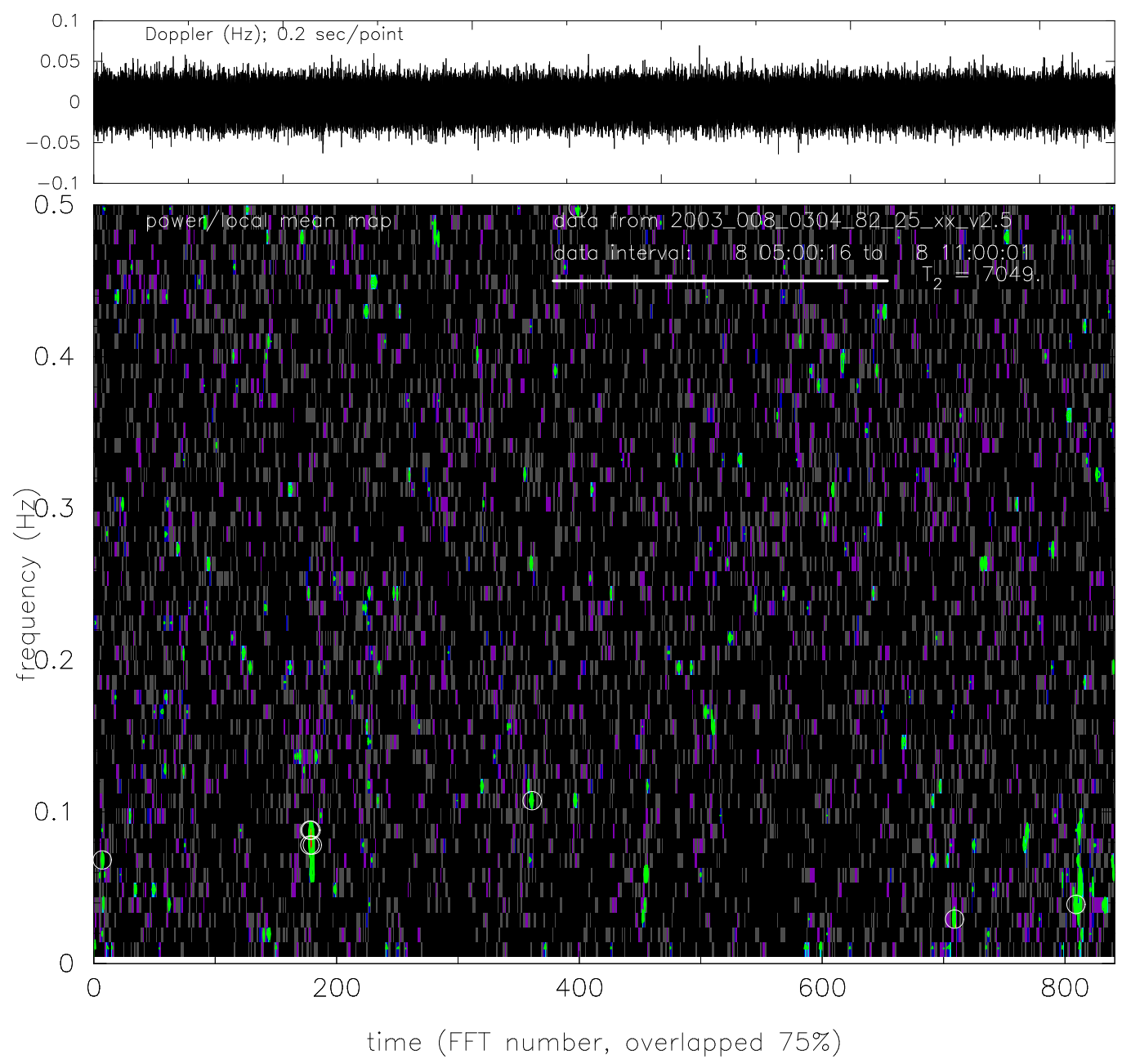

Figure 18: As for Figure 17, but for the Cassini two-way X-band track on 2003 DOY 008. Note the absence of high-contrast features near (08:50 UT, 0.22 Hz).

to analyze the time series in different ways to bring out different aspects. Doppler tracking data sets are not impossibly large: It is practical for a person to actually look at all the data with varying time-frequency resolution - in addition to using formal and automated analysis procedures. (A potential difficulty is still actually recognizing unanticipated features if they are present. Reference [77] has interesting discussions of the problems of recognizing unexpected things.) As emphasized by Thorne [108], the largest events may be from unexpected sources so the data analysis scheme must be robust enough that unexpected signals are not preprocessed away. 


\section{Detector Performance}

\subsection{Observations to date}

Table 3 lists spacecraft Doppler GW observations to date and observations possible in the near future.

Table 3: Spacecraft Doppler gravitational-wave observations. MO: Mars Observer; GLL: Galileo; ULS: Ulysses; MGS: Mars Global Surveyor; AMC: Advanced Media Calibration system (tropospheric calibration). A "pass" is a tracking pass over a given DSN antenna, i.e., about 8 hours long.

\begin{tabular}{|c|c|c|c|}
\hline Year & Spacecraft & Comment & Reference \\
\hline 1980 & Voyager & several passes; S-band uplink, S/X downlink; burst search & {$[60]$} \\
\hline 1981 & Pioneer 10 & $\begin{array}{l}3 \text { passes; long } T_{2} ; \mathrm{GW} \text { background limit; observationally } \\
\text { excluded GW from Geminga }\end{array}$ & {$[4,6]$} \\
\hline 1983 & Pioneer 11 & 3 days; long $T_{2} ;$ broadband periodic search & {$[18]$} \\
\hline 1988 & Pioneer 10 & 10 days; long $T_{2} ;$ chirp wave search & {$[5]$} \\
\hline 1990 & Ulysses & 3.5 days; short $T_{2}$ & {$[31,32]$} \\
\hline 1992 & Ulysses & $\begin{array}{l}14 \text { days; long } T_{2} \text {; periodic and chirp search; S-band uplink, } \\
\mathrm{S} / \mathrm{X} \text { band downlink }\end{array}$ & {$[31,32]$} \\
\hline 1993 & $\mathrm{MO} / \mathrm{GLL} / \mathrm{ULS}$ & $\begin{array}{l}19 \text { days; } \mathrm{X} / \mathrm{X} \text {-band on } \mathrm{MO} \text {; coincidence experiment; search } \\
\text { for all waveforms }\end{array}$ & {$[63,64,13]$} \\
\hline $1994-95$ & Galileo & 40/40 days (two oppositions); long $T_{2} ; \mathrm{S} / \mathrm{S}$-band & {$[3,13]$} \\
\hline 1997 & MGS & 21 days; short $T_{2} ; \mathrm{X} / \mathrm{X}$-band & [9] \\
\hline $2001-03$ & Cassini & $\begin{array}{l}40 / 40 / 20 \text { days (three oppositions); long } T_{2} ; \text { Ka-band; } \\
\text { AMC; multi-link plasma calibration }\end{array}$ & {$[19,78]$} \\
\hline $2016+$ & Juno & $\begin{array}{l}\text { opposition in early } 2016 \text {; long } T_{2} ; \mathrm{Ka} \text {-band; AMC; good } \\
\text { geometric coupling to galactic center }\end{array}$ & {$[33,83]$} \\
\hline $2019+$ & BepiColombo & $\begin{array}{l}\text { short } T_{2} ; \text { multi-link plasma calibration; AMC; good geo- } \\
\text { metric coupling to galactic center }\end{array}$ & {$[66,25]$} \\
\hline
\end{tabular}

The observations in Table 3 reflect increasing sensitivity between about 1980 and the early 2000s. These improvements were due both to engineering advancements (in spacecraft and in the DSN) and to programmatic decisions allowing use of planetary spacecraft for these observations. Voyager sensitivity was limited by a combination of plasma noise in the S-band uplink (see Figure 10) and spacecraft buffeting noise from its thrusters. Although the data volume was small, those observations were used in the first formal search for low-frequency burst waves [60]. The Pioneer spacecraft were spin-stabilized, resulting in lower spacecraft buffeting noise, but were again sensitivity-limited by plasma noise in the S-band radio links. Despite this, Pioneer data were able to observationally exclude putative sinusoidal GW emission from Geminga [4] and place the thenbest limit on a low-frequency GW background [6]. Ulysses observations in 1992 were the longest to date, motivated innovations in signal processing, and resulted in the then-best sensitivity to periodic and chirp waveforms [31, 32]. Mars Observer was the first spacecraft to have X-band on both the up- and downlinks, resulting in much-reduced plasma noise. Mars Observer, Galileo, and Ulysses did the first (and so far only) coincidence experiment [63, 13] which was used successfully to disqualify an event which was formally significant in one time series. Galileo observations in 1994-1995 had a long two-way light time and thus better GW response at lower Fourier frequencies. Unfortunately, the failure of the high-gain antenna required S-band only observations (thus high plasma noise) [9]. Mars Global Surveyor observations in 1997 were done with X-band links 
(but off of solar opposition) and were the only observations where spacecraft engineering telemetry was used to correct the Doppler data for the (slow) systematic spacecraft motion. Those data also showed strong correlation at the two-way light time, indicating the importance of tropospheric calibration and placing upper limits on antenna mechanical noise [9, 22].

Most of the sensitivity discussion in this paper, however, relates to Cassini observations. Cassini was launched on a mission to Saturn in 1997 [37]. After earth, Venus, and Jupiter gravity-assists, it continued on a free interplanetary cruise trajectory toward orbit insertion at Saturn. The Cassini gravitational-wave observations consisted of two 40-day data-taking campaigns, centered on the spacecraft's solar oppositions during 2001-2002 and 2002-2003, and one 20-day observation taken somewhat off opposition during late 2003. This data set is distinguished by its very sophisticated multi-link radio system (allowing essentially perfect plasma correction [69, 122, 70, 29, 121]) and by the Advanced Media Calibration system (allowing excellent tropospheric scintillation removal) [94, 96, 19, 76].

\subsection{Near-future observations}

There are two potentially-good-sensitivity near-future GW opportunities using Juno and BepiColombo in their cruise phases. Juno will reach Jupiter in July 2016. BepiColombo is a low-altitude orbiter of Mercury.

The Juno spacecraft has Ka-band up- and downlink, thus good immunity to plasma scintillation noise. Juno's two-way light time near opposition in 2016 will be about 4360 seconds, smaller than that of Cassini (thus Juno will, in sky-average, have poorer low-frequency response). Juno is distinguished, however, in that its GW coupling to the galactic center (the nearest plausible source of strong millihertz GWs) is much better than that of Cassini. For Juno, near the 2016 solar opposition, the cosine of the angle between the earth-spacecraft vector and the wavevector for a source at the galactic center is $\mu=\hat{k} \cdot \hat{n} \simeq 0.065$ [Eq. (1)]. Juno's transfer function to GWs (along with that of BepiColombo; see below) is shown in Figure 19); Juno will have substantially better geometric response to the galactic center in the $0.0001-0.001 \mathrm{~Hz}$ band, in particular.

The BepiColombo spacecraft will have a multi-link radio system similar to Cassini's. Thus plasma noise can be solved-for and removed from the data prior to GW analysis. The nominal launch is in January 2017. ${ }^{10}$ Because BepiColombo is an inner solar system mission, the twoway light time during the cruise phase will never be more than about 1700 seconds. There is a GW observation opportunity, however. Between about 1000 and 1100 days after launch (e.g., late 2019 to early 2020 for the nominal launch date) the two-way light time is $\simeq 1600 \mathrm{~s}$ while the earth-spacecraft vector changes orientation substantially on the celestial sphere. Thus the geometric coupling to sources on the sky changes. For example, $\theta=\arccos (\mu)$ for a source at the galactic center changes between about 150 and 50 degrees over $\simeq 100$ days. Selected intervals in this time window could be used to search for shorter GW bursts, for example, targeting specific geometrically-favorable directions (Figure 19 shows the coupling to the galactic center for late 2019, assuming nominal launch date.) Such a campaign would require the spacecraft's solar-electric propulsion to be turned off during GW observations.

\subsection{Sensitivity to periodic and quasi-periodic waves}

\subsubsection{Sinusoidal waves and chirps}

Sinusoidal sensitivity is traditionally stated as the amplitude $h$ of a sinusoidal $\mathrm{GW}$ required to achieve a specified SNR, as a function of Fourier frequency [18, 113, 16]. Conventionally, the

10 Trajectory information for BepiColombo's nominal mission was kindly provided by L. Iess and L. Imperi. 


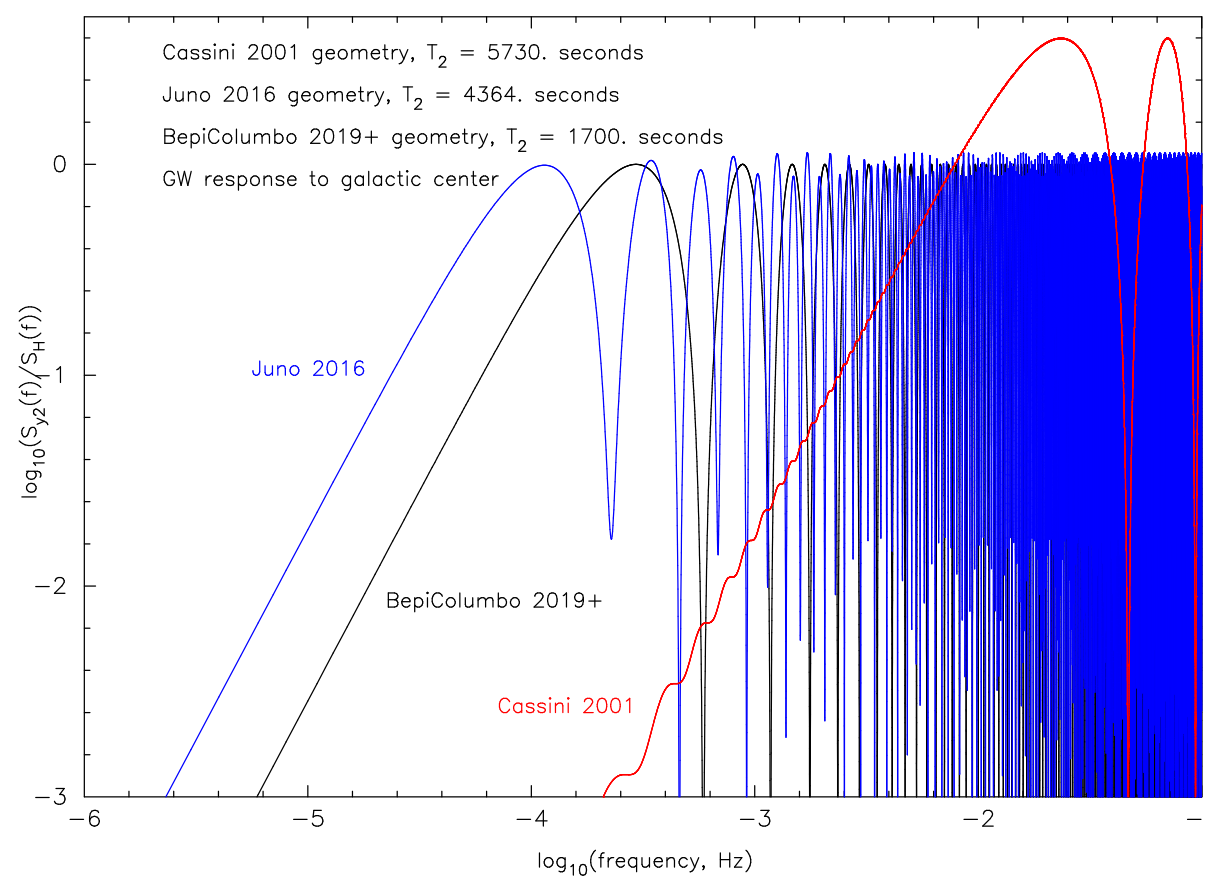

Figure 19: Power response of the Doppler system to a gravitational-wave signal, as a function of Fourier frequency, for signals from the direction of the galactic center. Blue: Juno 2016 opportunity. Black: BepiColombo 2019+ opportunity. Red: Cassini 2001 geometry.

signal is averaged over the sky and over polarization state $[52,127,16]$. Figure 20 shows the allsky sensitivity based on a smoothed version of the actual spectrum (black curve) for the Cassini 2001-2002 observation. Cassini achieves $\sim 10^{-16}$ all-sky sensitivity over a fairly broad Fourier band.

Early searches for periodic waves involved short duration observations (several hours to a few days; see, e.g., $[4,18])$ and thus ignorable modulation of an astronomical sinusoid due to changing geometry (non-time-shift-invariance of the GW transfer function; see Section 3). A true fixed-frequency signal would be reflected in the spectrum of the (noisy) Doppler times series as a "Rice-squared" random variable [97] at the signal frequency. Subsequent observations were over 10 - 40 days and the time dependence of the earth-spacecraft-source geometry became important: A sinusoidal excitation would be modulated into a non-sinusoidal Doppler response with power typically smeared over a few Fourier frequency resolution bins. Non-negligible modulation has both advantages and disadvantages. An advantage is that a real GW signal has a source-locationdependent signature in the data and this can be used to verify or refute an astronomical origin of a candidate. The disadvantage is that a simple spectral analysis is not sufficient for optimum detection (SNR losses of the simple spectral analysis technique are frequency and geometry dependent but can be $\simeq 3 \mathrm{~dB}$ or more in some observations) and the computational cost to search for even a simple astronomical-origin sinusoid becomes larger. Searches to date have addressed this with a hierarchical approach to the data analysis. First a suboptimal-but-simple spectral analysis is done. Candidates are then identified using an SNR threshold which is high enough to exclude Fourier components which, even with proper analysis, would be too weak to be classified as otherthan-noise. The idea is to use a computationally inexpensive procedure (i.e., FFTs) to exclude candidates which could not, in principle, be raised to a reasonable threshold SNR even with accurate matched filtering. The frequencies of candidate signals passing the threshold are saved and 


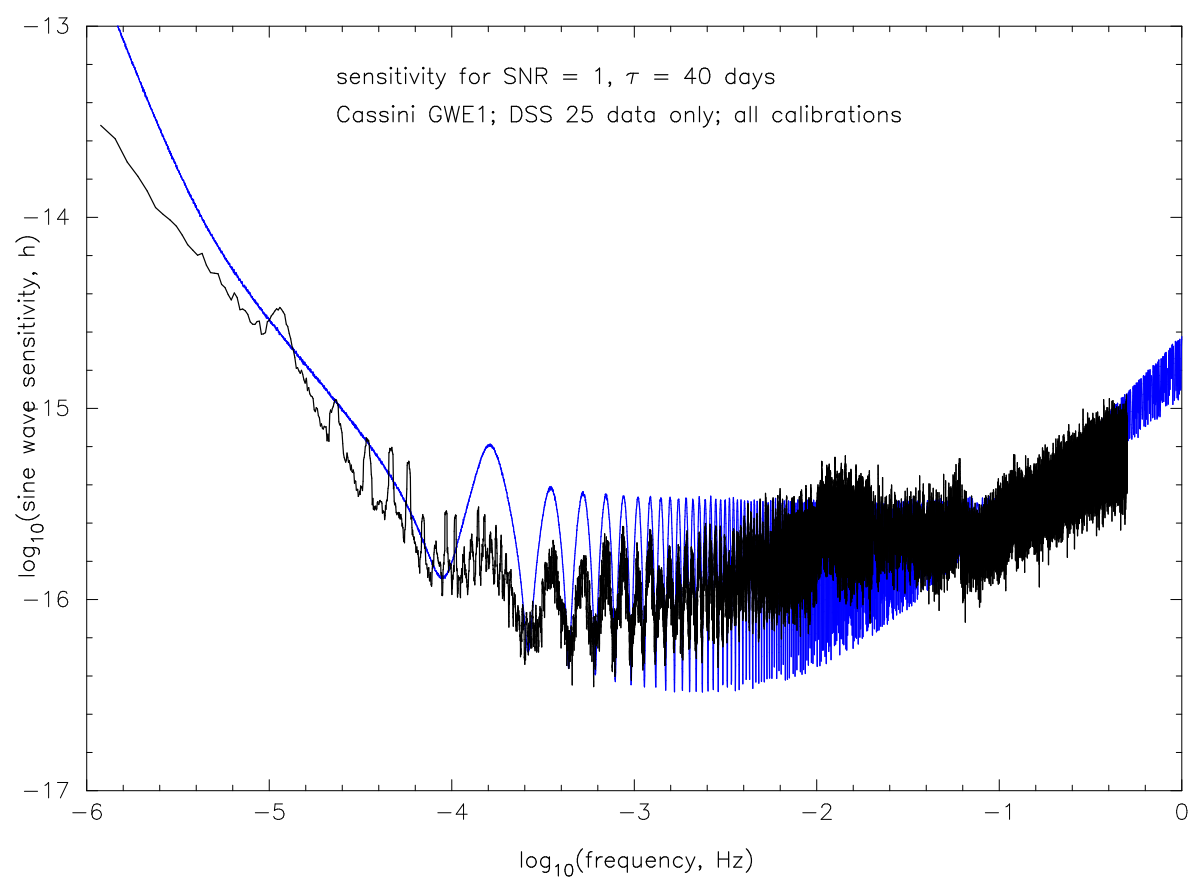

Figure 20: Sensitivity of the Cassini 2001-2002 gravitational wave observations, expressed as the equivalent sinusoidal strain sensitivity required to produce $\mathrm{SNR}=1$ for a randomly polarized isotropic background as a function of Fourier frequency. This reflects both the levels, spectral shapes, and transfer functions of the instrumental noises (see Section 4) and the GW transfer function (see Section 3). Black curve: sensitivity computed using smoothed version of observed noise spectrum; blue curve: sensitivity computed from pre-observation predicted noise spectrum [111].

matched filters are constructed using the known time-dependence of the earth-spacecraft geometry and for 20 points on the sky (the vertices of a dodecahedron projected onto the celestial sphere. ${ }^{11}$ )

Linear chirp processing adds an additional parameter (chirp rate) and requires detection thresholds to be set higher. In both the sinusoidal and chirp analyses, the pdfs of signal power have been used to assess candidates (see, e.g., [18, 3, 32]). Non-linear chirps, if source parameters are favorable, could be strong candidate signals. Analysis methods, anticipated sensitivity, and detection range for Cassini are discussed in [30].

\subsubsection{Nonsinusoidal periodic waves}

Calculations of Doppler response to GWs from a nonrelativistic binary system [127] show that the observed Doppler waveform can have a rich harmonic content. Monte Carlo calculations of GW strength from stars in highly elliptical orbits around the Galaxy's central black hole have been given in [53]. For these stellar-mass secondaries generate wave amplitudes more than an order of magnitude weaker than Cassini-era Doppler sensitivity.

\footnotetext{
11 This was suggested by Estabrook as giving "noncommittal" directions on the celestial sphere and have separations which are reasonably matched to the effective angular response of a typical Doppler tracking observation.
} 


\subsection{Burst waves}

The first systematic search for burst radiation was done by filtering the data to de-emphasize the dominant noise (plasma noise) relative to components of the time series anticorrelated at the two-way light time [60]. Analysis of subsequent data sets used matched filtering with assumed waveforms and targeted-sky-directions $[7,63,64]$. The utility of multiple-spacecraft observations for burst searches was discussed by $[28,112,13]$. Figure 21 is the crudest measure of currentgeneration (Ka-band, tropospheric corrected) all-sky burst sensitivity. It shows the power spectrum of two-way Doppler divided by the isotropic GW transfer function (see, e.g., [52, 54] and Section 5.4) computed as [108] $h_{\mathrm{c}}(f)=\left[2 f S_{y 2}^{\mathrm{gw}} / \bar{R}_{2}(f)\right]^{1 / 2}$, where $\bar{R}_{2}(f)$ is the sky- and polarization-averaged GW response function $[52,54,19]$. The best sensitivity, $h_{\mathrm{c}}<2 \times 10^{-15}$, occurs at about $0.3 \mathrm{mHz}$, set by the minimization of the antenna mechanical noise through its transfer function, the bandwidth, and the average coupling of the GW to the Doppler, $\bar{R}_{2}$, at this frequency.

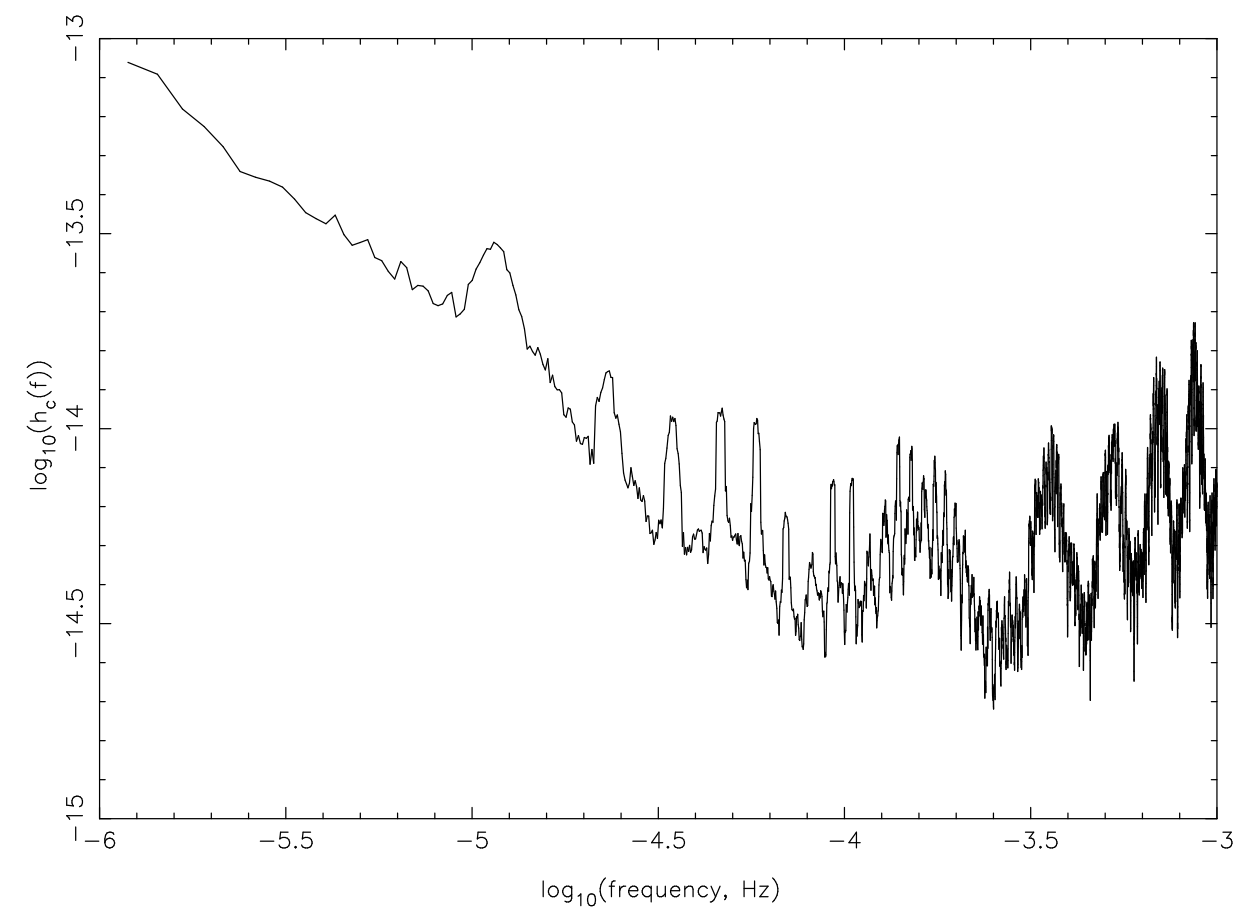

Figure 21: Characteristic all-sky strain sensitivity for a burst wave having a bandwidth comparable to center frequency for the Cassini 2001-2002 data set [19]. This is the crudest measure of sky-averaged burst sensitivity: the square root of the product of the Doppler spectrum and the Fourier frequency, divided by the sky-averaged GW response (see Section 6.4).

Sensitivity is not uniform over the sky and one can often do much better with knowledge of the direction-of-arrival or the waveform. Figure 22 shows contours of constant matched filter output for a circularly polarized mid-band burst wave using the Cassini solar opposition geometry of November 2003. The red dot shows the right ascension and declination of Cassini as viewed from the earth, the black dots are the positions of members of the Local Group of galaxies (larger dots indicating nearer objects), and "GC" marks the location of the galactic center. Contour levels are at $1 / 10$ of the maximum, with red contours at 0.9 to 0.5 of the maximum filter output and blue contours at 0.4 to 0.1 . The response is zero in the direction and anti-direction of the earth-Cassini vector (see Eq. (1)). The angular response changes for GWs in the long-wavelength limit. Figure 23 similarly shows matched filter signal output contours, but for a burst wave with 
characteristic duration $>T_{2}$. Pilot analyses using simplified waveforms [63, 11] have been done accounting for the local non-stationarity of the noise and varying assumed source position on the sky.

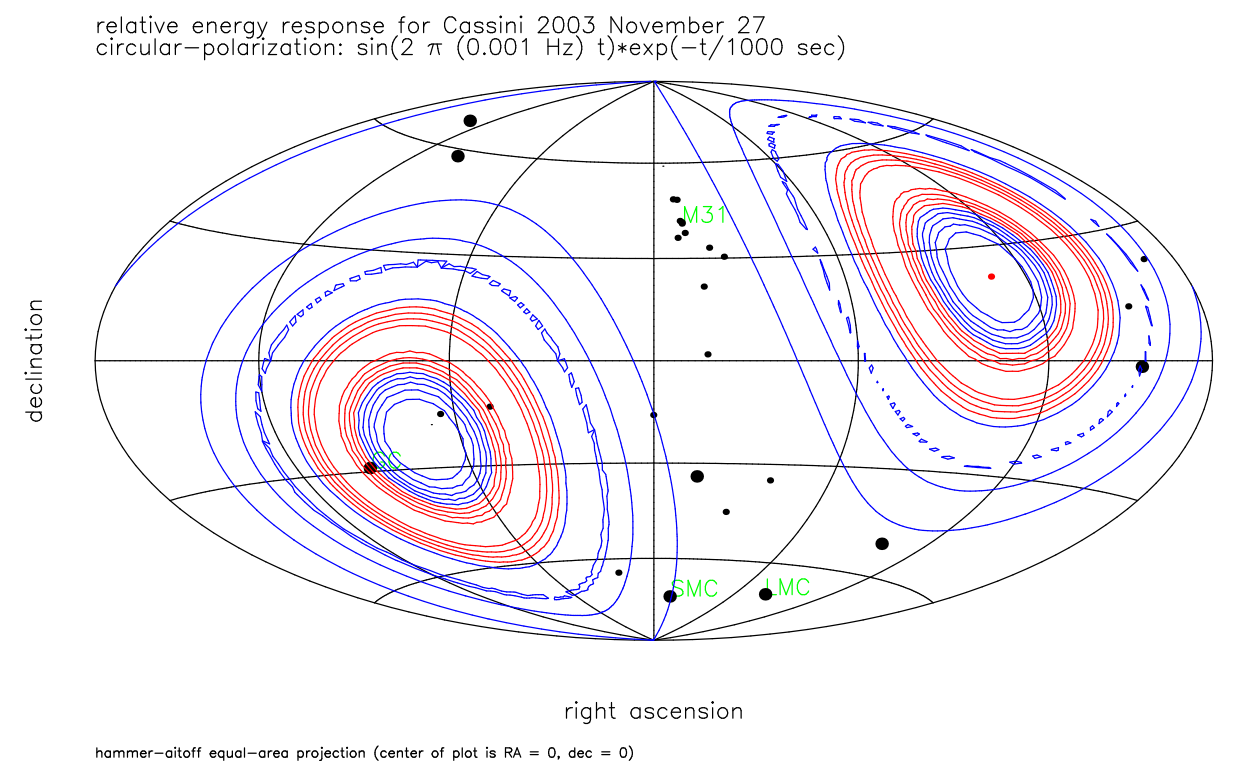

Figure 22: Contours of constant matched filter output for a wave having $h_{+}(t)=\sin (2 \pi t$. $0.001 \mathrm{~Hz}) \exp (-t / 1000 \mathrm{~s}) H(t)$ and $h_{\times}(t)$ its Hilbert transform, adapted from [64]. Cassini November 2003 geometry is assumed (the red dot is the right ascension and declination of Cassini). Black dots are the positions of members of the Local Group of galaxies. "GC" marks the location of the galactic center. Contour levels are at $1 / 10$ of the maximum, with red contours at 0.9 to 0.5 of the maximum signal output and blue contours at 0.4 to 0.1. Doppler response is zero in the direction of Cassini (and its anti-direction).

Waves from coalescing binary sources are intermediate between periodic and burst waves. Expected sensitivity and analysis methods have been treated in detail by Bertotti, Iess, and Vecchio [30, 124]; supermassive black hole coalescences with favorable parameters are visible with Cassini-class sensitivity out to $100 \mathrm{~s}$ of Mpc. Cassini is also sensitive to $\simeq 50 M_{\odot}$ intermediatemass black holes coalescing with the supermassive black hole at the galactic center [30].

\subsection{Sensitivity to a stochastic background}

A stochastic background of low-frequency GWs, potentially detectable with single or multiple spacecraft Doppler tracking, has been discussed by [52, 26, 58, 82, 6, 28, 44, 7, 54, 19]. The level of stochastic GWs is conventionally expressed either as the energy density in GWs relative to closure density, $\Omega$, as a characteristic rms strain, or as spectrum of strain. The best directly observational upper bounds on stochastic GWs in the low-frequency band come from the Cassini data. Details of how the upper limits were produced are given in [19]. Figure 24 shows limits to $\Omega$ as a function of Fourier frequency (upper limits expressed as spectrum of strain are given in [19]). The lowest bound is at $1.2 \times 10^{-6} \mathrm{~Hz}: \Omega<0.025$. Between $1.2 \times 10^{-6}$ and $\simeq 10^{-5} \mathrm{~Hz}, \Omega<0.1$, while between about $10^{-5}-10^{-4} \mathrm{~Hz}$ the upper bounds are between 0.1 to about 1.0. For $f>10^{-4} \mathrm{~Hz}$ the limits to $\Omega$ are larger than 1 . The Cassini data improved limits to $\Omega$ in the $10^{-6}$ to $10^{-4} \mathrm{~Hz}$ band by factors of 500-1200 (depending on Fourier frequency) compared with earlier Doppler experiments.

Predictions for an astrophysical GW background in the low-frequency band, e.g., from an ensemble of galactic binary stars or an ensemble of massive black hole binaries, have mainly been 


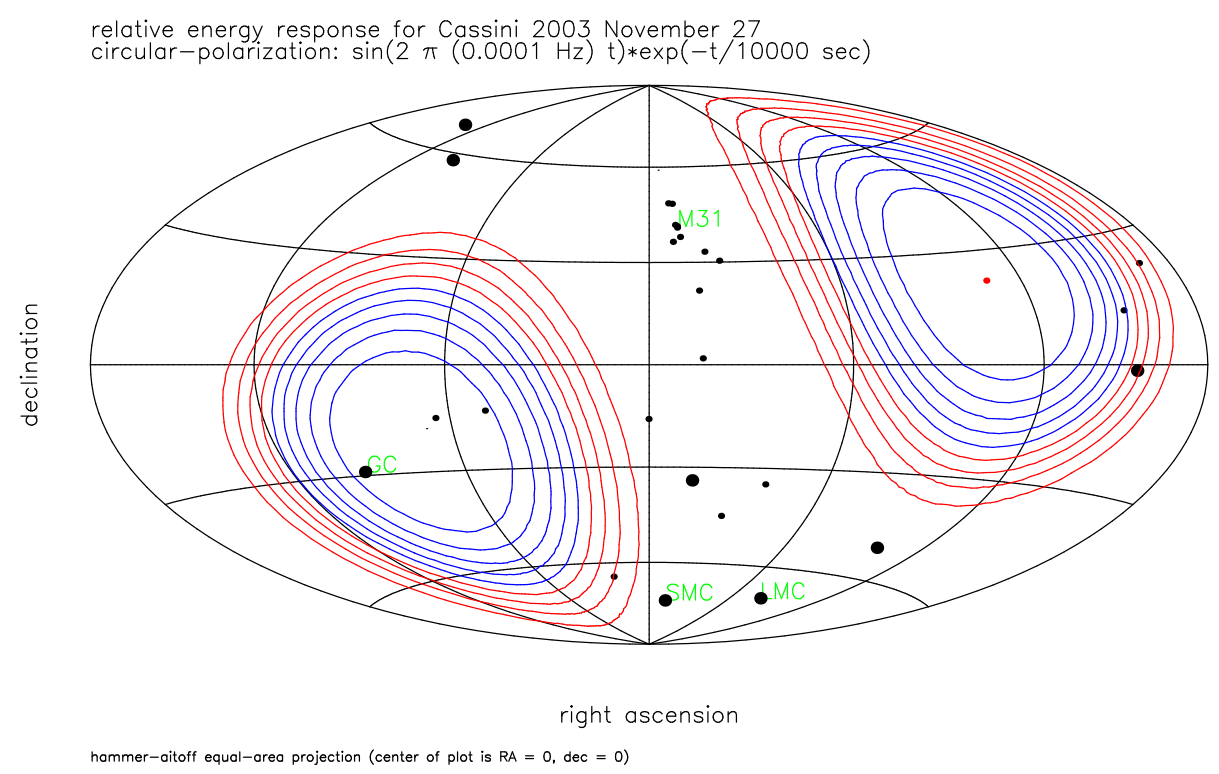

Figure 23: As in Figure 22 but for a wave with $h_{+}(t)=\sin (2 \pi t \cdot 0.0001 \mathrm{~Hz}) \exp (-t / 10000 \mathrm{~s}) H(t)$ and $h_{\times}(t)$ its Hilbert transform. This model waveform is long compared with $T_{2}$.

aimed at the design sensitivity of future dedicated GW missions [24]. The galactic binary star background is much too weak to be seen with spacecraft Doppler tracking. At lower frequencies $\left(10^{-9}-10^{-6} \mathrm{~Hz}\right)$ the strength of a GW background from an ensemble of coalescing black hole binaries has been estimated [93, 72, 132] mostly in the context of a pulsar timing array. Extrapolations or predictions in the low-frequency band [72, 132] give strengths substantially lower than spacecraft Doppler tracking can presently observe (see Figure 21). 


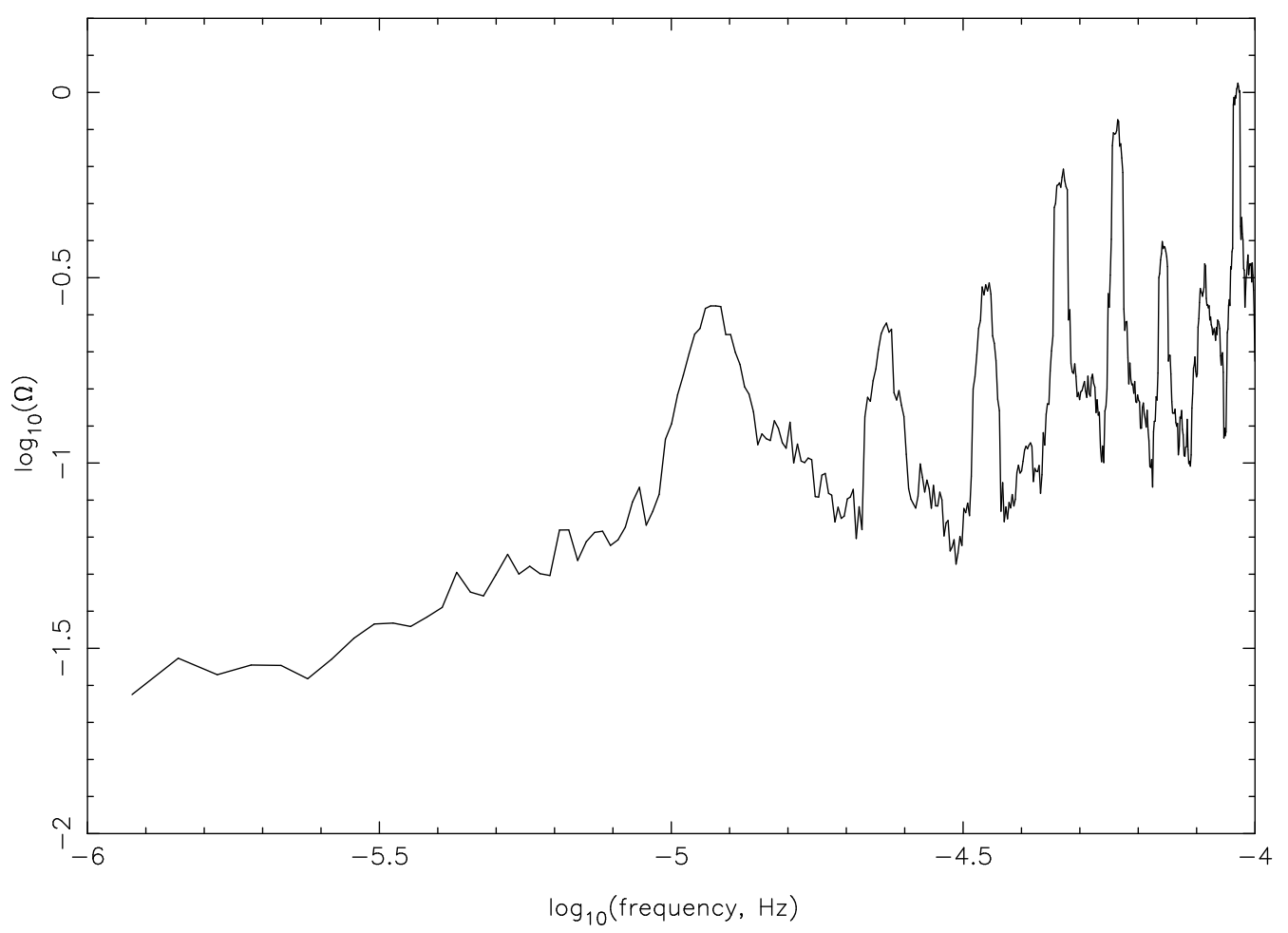

Figure 24: Upper limits to the energy density of GWs in bandwidth equal to center frequency, relative to closure energy density. This assumes an isotropic GW background, $H_{0}=75 \mathrm{~km} \mathrm{~s}^{-1} \mathrm{Mpc}^{-1}$, and is computed from the Cassini 2001-2002 data [19].

\section{Improving Doppler Tracking Sensitivity}

What would be required to improve broadband ${ }^{12}$ burst sensitivity ten-fold, to $\simeq 2 \times 10^{-16}$ (thus, in a 40 day observation, have sensitivity to periodic waves of $\simeq 10^{-17}$ )? Assuming that there is not some unexpected systematic effect entering between $10^{-15}$ and $10^{-16}$ and that the noises are independent (thus variances add and each component must be brought to $\simeq 10^{-16}$ ), Table 4 shows the required improvements in the principal subsystems.

FTS stability at the $10^{-17}$ level for $\tau \simeq 1000 \mathrm{~s}$ has been demonstrated [62]. (If high-stability flyable frequency standards become available in the future, they would allow simultaneous multiple one- and two-way Doppler measurements. These multiple observations would give excellent diagnostics of many instrumental noises and provide further rejection of systematic effects [125, 8, 117]). Better frequency standards would require better frequency distribution. Prototypes for frequency distribution within the Atacama Large Millimeter Array achieve stability $10^{-16}$ or better for time scales of about 1000 seconds [39].

Improving ground electronics noise by $2 \mathrm{X}$ is probably possible by even more careful design. Reducing tropospheric scintillation by $10 \mathrm{X}$ will require either an antenna at very high altitude, improvements in AMC technology (e.g., exactly coincident beams, better water vapor radiometry technology), or perhaps an interesting idea (suggested independently by Estabrook [48] and Hellings [59]) whereby a second ground station (listen-only, at high altitude) could be employed

12 Signal processing procedures which exploit differences in the signal and noise transfer functions can give improved sensitivity at selected frequencies (see, e.g., [110, 9, 11]). 
Table 4: Required improvement in subsystems to improve overall Doppler sensitivity by a factor of 10 relative to Cassini-era performance.

\begin{tabular}{lll}
\hline Noise source & Comment $\left(\sigma_{y}\right.$ at $\left.\tau=1000 \mathrm{~s}\right)$ & $\begin{array}{l}\text { Required } \\
\text { improvement }\end{array}$ \\
\hline Frequency standard & currently FTS + distribution $\simeq 8 \times 10^{-16}$ & $\simeq 8 \mathrm{X}$ \\
Ground electronics & currently $\simeq 2 \times 10^{-16}$ & $\simeq 2 \mathrm{X}$ \\
Tropospheric scintillation & currently $\simeq 10^{-15}$ under favorable conditions & $\simeq 10 \mathrm{X}$ \\
Plasma scintillation & Cassini-class radio system probably adequate for & $\simeq 1 \mathrm{X}$ \\
& calibration to $\simeq 10^{-16}$ & \\
Spacecraft motion & currently $\simeq 2 \times 10^{-16}$ & $\simeq \mathrm{X}$ \\
Antenna mechanical & currently $\simeq 2 \times 10^{-15}$ under favorable conditions & $\simeq 20 \mathrm{X}$ \\
\hline
\end{tabular}

to synthesize a Doppler observable which has the (presumably much lower) tropospheric phase scintillation noise of the higher-altitude receive-only station. Plasma scintillation correction technology is already adequate to reach an Allan deviation of $\sim 10^{-16}$. Cassini spacecraft unmodeled motion was measured to be within a factor of about 2 of $10^{-16}$ (see Section 4.8); it is not clear what actually limited the Cassini motion measurement so additional analysis/design might be required to assure that this component entered at the $10^{-16}$ level or lower.

The largest required improvement is in antenna mechanical noise. It is impractical to build a large, steel, earth-based, moving structure (such as a $34-\mathrm{m}$ antenna) which has intrinsic $\simeq 10^{-16}$ mechanical stability; performance at this level will probably require a separate calibration/removal of mechanical noise. One suggestion is to exploit the differing transfer function of antenna mechanical noise to two- and three-way observations [15]. Suppose that a stiffer (that is, smaller mechanical noise) ancillary antenna is co-located with the two-way tracking antenna. The ancillary antenna takes data in the "listen-only" (three-way) mode. The desired Doppler signal, $y_{s}$, and mechanical noises of the two antennas enter the time series of fractional Doppler fluctuation according to

$$
\begin{aligned}
& y_{2}(t)=M_{2}(t)+M_{2}\left(t-T_{2}\right)+y_{s} \\
& y_{3}(t)=M_{3}(t)+M_{2}\left(t-T_{2}\right)+y_{s}
\end{aligned}
$$

where $M_{2}$ and $M_{3}$ are the time series of mechanical noise at the two and three-way stations. The data combination [15]

$$
E(t)=y_{3}(t)+y_{3}\left(t-T_{2}\right)-y_{2}\left(t-T_{2}\right)=M_{3}(t)+M_{3}\left(t-T_{2}\right)+y_{s}
$$

has the signal content of the standard two-way observation but antenna mechanical noise

as if the ancillary antenna were both transmitting and receiving. If the ancillary antenna is sufficiently stiff (i.e., if the magnitude of $M_{3}$ is small compared with the magnitude of $M_{2}$ ) then mechanical noise in the observation can in principle be reduced substantially.

This idea was tested during an otherwise-routine Cassini observation [15]. The Cassini spacecraft was tracked in the conventional two-way mode using NASA's DSS14 70-m station while simultaneous three-way data were taken at a nearby antenna (DSS 25). During the track DSS 14's subreflector was deliberately articulated to introduce a large, artificial "antenna mechanical variation" (the signal path within the antenna was described in Section 4). Figure 25 shows the twoand three-way Doppler time series during the test. The upper panel shows the "two-pulse" signature of antenna mechanical variation in the two-way data (Eq. (6) and Figure 7). The lower panel shows the effect of the deliberate subreflector motion in the three-way Doppler [Eq. (7)]. 


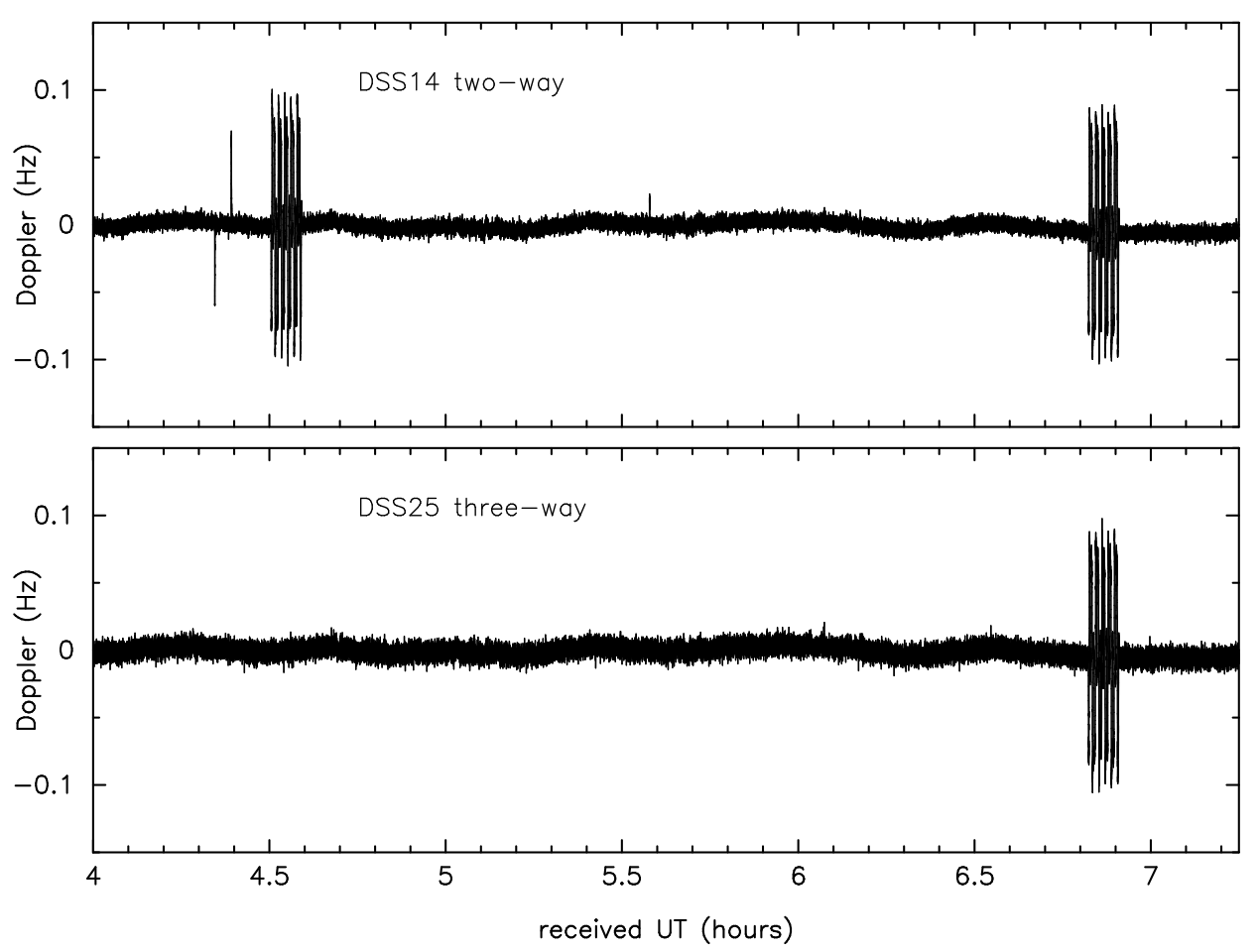

Figure 25: Time series of DSS 14 two-way (upper panel) and DSS 25 three way (lower panel) Doppler during the 2007 March 15 antenna mechanical noise test. At about 04:30 UT the subreflector at DSS 14 was deliberately articulated to produce large antenna mechanical noise variation. The effect in the twoway Doppler is seen immediately and at a two-way light time $\left(T_{2}=8341.6 \mathrm{~s}\right)$ later (see Figure 7$)$. The receive-only three-way station is unaffected at 04:30 UT (it is receiving a signal transmitted a two-way light time earlier) but observes the effect of the deliberate subreflector motion a two-way light time later (lower panel). (The three impulsive glitches in the two-way time series are unrelated to this mechanical noise test.) Figure adapted from [15].

Figure 26 shows a blowup of the time series of the two-way Doppler during the subreflector motion event (upper panel) and the data combination $E(t)$ formed using the two- and three-way data. The two-way mechanical variability cancels to the level of other noises.

Suitably stiff antennas (i.e., antennas with mechanical stability at least an order of magnitude better than that of DSS 25) have been built for radio astronomy applications [103]. These or comparable antennas could be used to reduce the antenna mechanical noise in Doppler gravitational-wave tracks to $\sim 10^{-16}$ or lower for $\tau=1000 \mathrm{~s}$. Of course one would not use this technique except in situations where the antenna mechanical noise dominates. Some considerations for a practical implementation of this method are discussed in [14].

There is no currently-planned mission that requires Doppler stability at the $\simeq 10^{-16}$ level. Indeed unless such stability can be achieved inexpensively that level of Doppler performance might have to be justified by a mission dedicated to precision radio science. As outlined above, however, $\sim 10^{-16}$ burst sensitivity may be possible with extensions of current technologies. To do several orders of magnitude better than $10^{-16}$, however - e.g., to achieve sensitivity adequate to detect the very weak GWs from known galactic binaries - would almost certainly require a different utilization of electromagnetic tracking [24], discussed briefly in Section 8. 


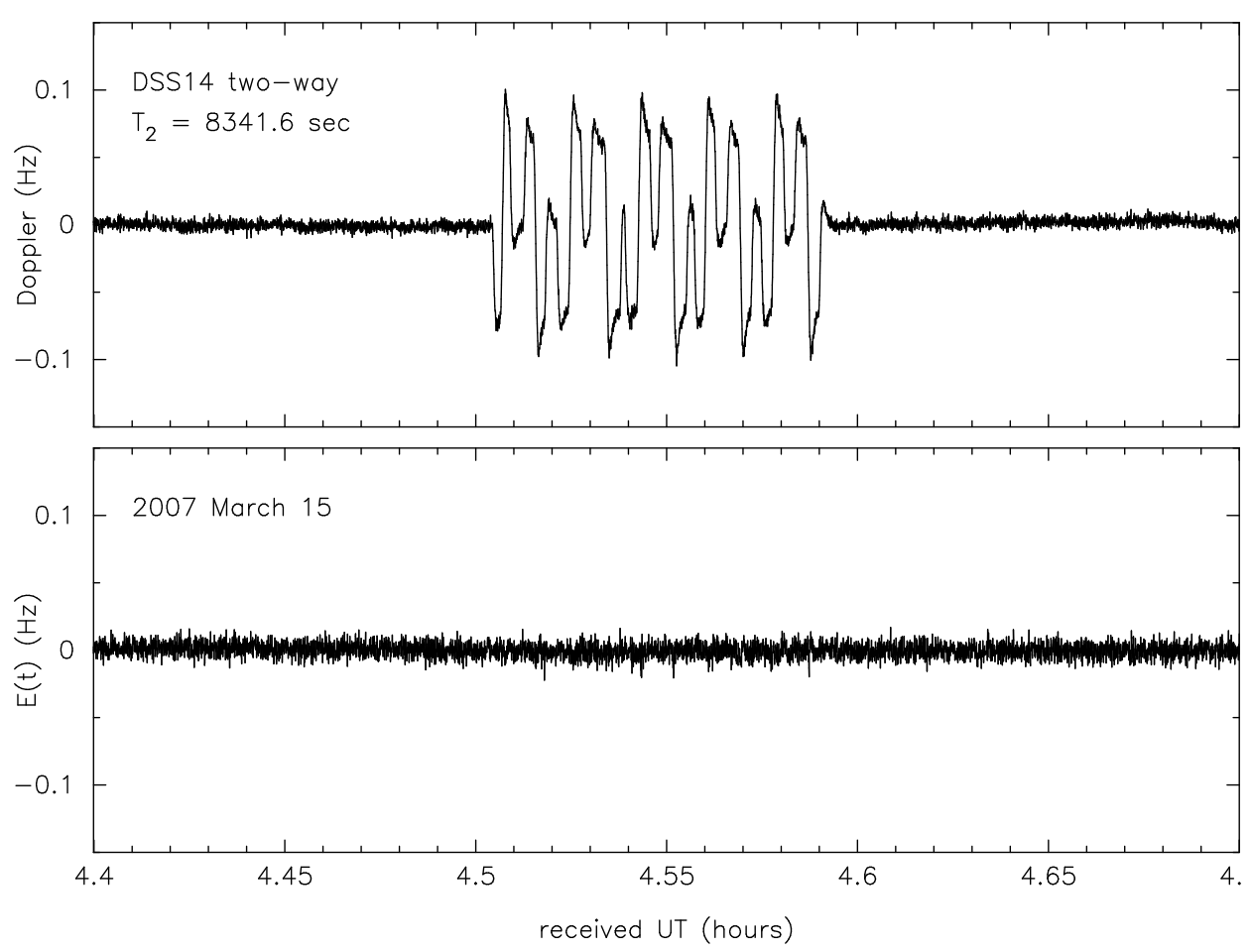

Figure 26: Blowup of DSS 14 two-way Doppler time series (upper panel) near the deliberate subreflector articulation. The lower panel shows the data combination $\mathrm{E}(\mathrm{t})$, which cancels the antenna mechanical noise in the two-way time series leaving the antenna mechanical noise of the three-way station (DSS 25) and other secondary noises. Figure adapted from [15].

\section{The LISA Low-Frequency Detector}

Current Doppler tracking observations piggy-back on spacecraft mainly serving the planetary science community. Future low-frequency detectors could be dedicated GW missions - fully spacebased - involving separated drag-free test masses [24, 105, 90, 115]. The LISA/eLISA (Laser Interferometer Space Antenna) mission is currently (2015) in the design and development stage, with a technology demonstration mission, LISA Pathfinder, launched in December 2015. The three LISA sciencecraft will form an approximately equilateral triangle with nominal $5 \times 10^{9} \mathrm{~m}$ armlengths (time-variable by $\sim 1 \%$ due to celestial mechanics). Six one-way laser-driven optical links between spacecraft pairs will monitor Doppler (or phase) fluctuations as the test masses respond to incident GWs. ${ }^{13}$ The principal advantages to moving all the apparatus to space are that the environment is very stable and drag-free technology can be employed. The final noise level can then in principle be set by (very small) optical-path and proof mass noises [24]. LISA's anticipated sensitivity is excellent: $\sim 10^{-23}$ for sinusoidal signals in a one year integration.

To reach the levels of the secondary optical-path and proof-mass noises, however, LISA must first cancel laser phase noise (which is otherwise overwhelming, $\simeq 160 \mathrm{~dB}$ larger than the secondary noises). Since LISA's armlengths cannot be made equal and constant, conventional laser noise cancelling methods, e.g., Michelson interferometry, will not work. LISA will use a technique based on the transfer functions of signals and noises to the inter- and intra-spacecraft Doppler data called

\footnotetext{
13 One-way tracking emphasizes the symmetry of the LISA array and simplifies the analysis of the apparatus; for technical reasons the actual implementation of LISA may involve some of the links being two-way [101].
} 
"time-delay interferometry" (TDI; see, e.g., [116]), to cancel the laser phase noises. ${ }^{14}$ TDI had its genesis in Doppler tracking where, as with LISA, time-of-flight of GWs and electromagnetic waves must be treated explicitly in the analysis.

14 TDI developed in increasing sophistication to account for unequal armlengths, differences between the (unequal) armlengths on given up- and down-links due to aberration, and time-dependences of the unequal, aberrated armlengths. For a discussion of this development see [116] and references therein. TDI also allows LISA's laser noises to be canceled in many ways $[17,51,118]$. In particular, one laser-noise-free combination is insensitive to GWs, but responds to the instrumental noises; this combination will be used to discriminate a stochastic GW background due to galactic binary stars from instrumental noises [114,45]. Because multiple laser-noise-free combinations can be simultaneously constructed, the optimum sensitivity of the LISA array can be achieved by appropriately linear combinations of the TDI data streams [92]. 


\section{Concluding Comments}

This paper discussed the principles of operation and status of spacecraft Doppler tracking, the current-generation GW detector technology in the $\sim 10^{-6}$ to $10^{-1} \mathrm{~Hz}$ band. Doppler tracking differs from all other currently-operating detectors in that the size of the apparatus (earth-spacecraft distance) is large compared with the GW wavelength. As a consequence times-of-flight of GWs and radio waves through the apparatus are important, resulting in a three-pulse signal response and various two-pulse noise responses. The different signal and noise transfer functions suggest data analysis approaches for various waveforms; some of these approaches were outlined here. The sensitivity of current-generation Doppler observations was discussed as well as what would be required to improve this sensitivity by another order of magnitude (to $\sim 10^{-17}$ for sinusoidal waves). Further large sensitivity improvements in the low-frequency band will require dedicated multispacecraft arrays in space. Until such a dedicated mission flys, spacecraft tracking will provide the best observational capability in the low-frequency GW band. 


\section{Acknowledgements}

The precision Doppler tracking capability described here resulted from work by many dedicated people at NASA, in the Mars Observer/Mars Global Surveyor, Ulysses, Galileo, and Cassini Projects (and the international partners of those Projects), the NASA/JPL Deep Space Network, and the JPL Technical Divisions. I am particularly indebted to B. Bertotti, F.B. Estabrook, L. Iess, R.V. Stachnik, M. Tinto, H.D. Wahlquist, and E.J. Weiler. The research described here was carried out at the Jet Propulsion Laboratory, California Institute of Technology, under a contract with the National Aeronautics and Space Administration. 


\section{References}

[1] Abbate, S. F. et al., "The Cassini gravitational wave experiment", in Cruise, M. and Saulson, P., eds., Gravitational-Wave Detection, Proc. of SPIE, 4856, pp. 90-97, (The International Society for Optical Engineering, New York, 2003). (Cited on pages 24 and 28.)

[2] Anderson, A. J., "Probability of Long Period Gravitational Radiation", Nature, 229, 547-548 (1971). [DOI]. (Cited on page 7.)

[3] Anderson, J. D., Armstrong, J. W., Campbell, J. K., Estabrook, F. B., Krisher, T. P. and Lau, E. L., "Gravitation and Celestial Mechanics Investigations with Galileo", Space Sci. Rev., 60, 591610 (1992). [DOI], [ADS]. (Cited on pages 37 and 40.)

[4] Anderson, J. D., Armstrong, J. W., Estabrook, F. B., Hellings, R. W., Lau, E. K. and Wahlquist, H. D., "Pioneer 10 search for gravitational waves - no evidence for coherent radiation from Geminga", Nature, 308, 158-160 (1984). [DOI], [ADS]. (Cited on pages 29, 37, and 39.)

[5] Anderson, J. D., Armstrong, J. W. and Lau, E. K., "Upper Limits for Gravitational Radiation from Supermassive Coalescing Binaries", Astrophys. J., 408, 287-292 (1993). [DOI], [ADS]. (Cited on pages $29,30,32,35$, and 37.)

[6] Anderson, J. D. and Mashhoon, B., "Pioneer 10 Search for Gravitational Waves - Limits on a Possible Isotropic Cosmic Background in the Microhertz Region", Astrophys. J., 290, 445-448 (1985). [DOI], [ADS]. (Cited on pages 37 and 42.)

[7] Armstrong, J. W., "Spacecraft Gravitational Wave Experiments", in Schutz, B. F., ed., Gravitational Wave Data Analysis, Proceedings of the NATO Advanced Research Workshop, held at Dyffryn House, St. Nichols, Cardiff, Wales, 6 - 9 July 1987, NATO ASI Series C, 253, pp. 153-172, (Kluwer, Dordrecht; Boston, 1989). (Cited on pages 29, 41, and 42.)

[8] Armstrong, J. W., "Gravitational Wave Search With the Clock Mission", in Maleki, L., ed., Gravitational Wave Search with the Clock Mission, Proceedings of the Workshop on the Scientific Applications of Clocks in Space, Pasadena, California, $7-8$ November 1996, JPL Publications, 97-15, pp. 33-40, (Jet Propulsion Laboratory, Pasadena, CA, 1997). (Cited on page 44.)

[9] Armstrong, J. W., "Radio Wave Phase Scintillation and Precision Doppler Tracking of Spacecraft", Radio Sci., 33, 1727-1738 (1998). [DOI]. (Cited on pages 17, 18, 20, 29, 31, 37, 38, and 44.)

[10] Armstrong, J. W., "Low-Frequency Gravitational Wave Searches Using Spacecraft Doppler Tracking", lecture notes, Caltech, (2000). URL (accessed 5 August 2005):

http://cajagwr.caltech.edu/pdf/Armstrong.pdf. (Cited on pages 16, 17, 20, and 21.)

[11] Armstrong, J. W., "The Cassini Gravitational Wave Experiment", lecture notes, Caltech, (2002). URL (accessed 5 August 2005):

http://cajagwr.caltech.edu/scripts/armstrong.ram. (Cited on pages 16, 17, 20, 21, 22, 30, 42, and 44.)

[12] Armstrong, J. W., "Doppler Tracking of Spacecraft for Gravitational Wave Detection in the LowFrequency Band", lecture notes, Caltech, (2002). URL (accessed 5 August 2005):

http://elmer.tapir.caltech.edu/ph237/week15/week15.html. (Cited on page 17.)

[13] Armstrong, J. W., Bertotti, B., Estabrook, F. B., Iess, L. and Wahlquist, H. D., "The Galileo/Mars Observer/Ulysses Coincidence Experiment", in Coccia, E., Veneziano, G. and Pizzella, G., eds., Gravitational Waves, Proceedings of the Second Edoardo Amaldi Conference on Gravitational Waves, CERN, 1-4 July 1997, pp. 159-167, (World Scientific, Singapore; River Edge, NJ, 1998). (Cited on pages $30,34,37$, and 41 .) 
[14] Armstrong, J. W., Estabrook, F. B., Asmar, S. W., Iess, L. and Tortora, P., "Reducing Antenna Mechanical Noise in Precision Doppler Tracking", IPN Progress Report, 2006, 42-165 (2006). URL (accessed 1 December 2007):

http://tmo.jpl.nasa.gov/progress_report/42-165/165F.pdf. (Cited on page 46.)

[15] Armstrong, J. W., Estabrook, F. B., Asmar, S. W., Iess, L. and Tortora, P., "Reducing antenna mechanical noise in precision spacecraft tracking", Radio Sci., 43, RS3010 (2008). [DOI], [ADS]. Online version (accessed 1 December 2007):

http://tmo.jpl.nasa.gov/progress_report/42-165/165F.pdf. (Cited on pages 45, 46, and 47.)

[16] Armstrong, J. W., Estabrook, F. B. and Tinto, M., "Time-Delay Interferometry for Space-Based Gravitational Wave Searches", Astrophys. J., 527, 814-826 (1999). [DOI], [ADS]. (Cited on pages 10, 11, 34, 38, and 39.)

[17] Armstrong, J. W., Estabrook, F. B. and Tinto, M., "Sensitivities of Alternate LISA Configurations", Class. Quantum Grav., 18, 4059-4065 (2001). [DOI], [ADS]. (Cited on page 48.)

[18] Armstrong, J. W., Estabrook, F. B. and Wahlquist, H. D., "A Search for Sinusoidal Gravitational Radiation in the Period Range 30-2000 seconds", Astrophys. J., 318, 536-541 (1987). [DOI]. (Cited on pages $11,29,30,34,37,38,39$, and 40 .)

[19] Armstrong, J. W., Iess, L., Tortora, P. and Bertotti, B., "Stochastic Gravitational Wave Background: Upper Limits in the $10^{-6}$ to $10^{-3} \mathrm{~Hz}$ Band", Astrophys. J., 599, 806-813 (2003). [DOI], [ADS]. (Cited on pages 17, 20, 21, 22, 24, 25, 27, 28, 29, 31, 37, 38, 41, 42, and 44.)

[20] Armstrong, J. W. and Sramek, R. A., "Observations of Tropospheric Phase Scintillations at $5 \mathrm{GHz}$ on Vertical Paths", Radio Sci., 17, 1579-1586 (1982). [DOI]. (Cited on pages 18, 20, and 28.)

[21] Armstrong, J. W., Woo, R. and Estabrook, F. B., "Interplanetary Phase Scintillation and the Search for Very Low Frequency Gravitational Radiation", Astrophys. J., 230, 570-574 (1979). [DOI], [ADS]. (Cited on pages 17, 18, and 20.)

[22] Asmar, S. W., Armstrong, J. W., Iess, L. and Tortora, P., "Spacecraft Doppler tracking: Noise budget and accuracy achievable in precision radio science observations", Radio Sci., 40, RS2001 (2005). [DOI]. (Cited on pages 14, 16, 17, 20, 21, 24, 28, and 38.)

[23] Barnes, J. A. et al., "Characterization of Frequency Stability", IEEE Trans. Instrum. Meas., 20, 105-120 (1971). [DOI]. (Cited on pages 9, 15, 16, 24, and 28.)

[24] Bender, P. L. et al. (LISA Study Team), LISA. Laser Interferometer Space Antenna for the detection and observation of gravitational waves. An international project in the field of Fundamental Physics in Space. Pre-Phase A report, MPQ-233, (Max-Planck-Institut für Quantenoptik, Garching, 1998). (Cited on pages $9,43,46$, and 47.)

[25] Benkhoff, J. et al., "BepiColumbo - Comprehensive Exploration of Mercury: Mission Overview and Science Goals", Planet. Space Sci., 58, 2-20 (2010). [DOI], [ADS]. (Cited on page 37.)

[26] Bertotti, B. and Carr, B. J., "The Prospects of Detecting Gravitational Background Radiation by Doppler Tracking Interplanetary Spacecraft", Astrophys. J., 236, 1000-1011 (1980). [DOI], [ADS]. (Cited on pages 30 and 42.)

[27] Bertotti, B., Comoretto, G. and Iess, L., "Doppler tracking of spacecraft with multi-frequency links", Astron. Astrophys., 269, 608-616 (1993). [ADS]. (Cited on page 18.)

[28] Bertotti, B. and Iess, L., "Doppler Search for a Gravitational Background Radiation with Two Spacecraft", Gen. Relativ. Gravit., 17, 1043-1058 (1985). [DOI], [ADS]. (Cited on pages 30, 41, and 42.) 
[29] Bertotti, B., Iess, L. and Tortora, P., "A test of general relativity using radio links with the Cassini spacecraft", Nature, 425, 374-376 (2003). [DOI], [ADS]. (Cited on pages 14, 18, 20, 21, 24, and 38.)

[30] Bertotti, B., Vecchio, A. and Iess, L., "Gravitational Waves From Coalescing Binaries and Doppler Experiments", Phys. Rev. D, 59, 082001 (1999). [DOI], [arXiv:gr-qc/9806021]. (Cited on pages 40 and 42.)

[31] Bertotti, B. et al., "The gravitational wave experiment", Astron. Astrophys. Suppl., 92, 431-440 (1992). [ADS]. (Cited on page 37.)

[32] Bertotti, B. et al., "Search for gravitational wave trains with the spacecraft ULYSSES", Astron. Astrophys., 296, 13-25 (1995). [ADS]. (Cited on pages 29, 37, and 40.)

[33] Bolton, S. J. et al. (Juno Science Team), "The Juno Mission", in Barbieri, C., Chakrabarti, S., Coradini, M. and Lazzarin, M., eds., Galileo's Medicean Moons: Their Impact on 400 Years of Discovery, IAU Symposium, S269, pp. 92-100, (Cambridge University Press, Cambridge; New York, 2010). [DOI], [ADS]. (Cited on page 37.)

[34] Bracewell, R., The Fourier Transform and Its Applications, (McGraw-Hill, New York, 1965). (Cited on page 9.)

[35] "CaJAGWR website", project homepage, Caltech. URL (accessed 5 August 2005): http://cajagwr.caltech.edu/. (Cited on page 7.)

[36] Camp, J. B. and Cornish, N. J., "Gravitational Wave Astronomy", Annu. Rev. Nucl. Part. Sci., 54, 525-577 (2004). [DOI], [ADS]. (Cited on page 7.)

[37] "Cassini-Huygens Mission to Saturn \& Titan", project homepage, Jet Propulsion Laboratory. URL (accessed 5 August 2005):

http://saturn.jpl.nasa.gov/home/. (Cited on pages 13, 14, and 38.)

[38] Chen, G. and Herring, T. A., "Effects of Atmospheric Azimuthal Asymmetry on the Analysis of Space Geodetic Data", J. Geophys. Res., 102(B9), 20489-20502 (1997). [DOI], [ADS]. (Cited on page 22.)

[39] Cliche, J.-F. and Shillue, B., "Precision Timing Control for Radioastronomy: Maintaining Femtosecond Synchronization in the Atacama Large Millimeter Array", IEEE Contr. Syst. Mag., 26, 19-26 (2006). [DOI]. (Cited on page 44.)

[40] Coles, W. A., "Interplanetary Scintillation", Space Sci. Rev., 21, 411-425 (1978). [DOI]. (Cited on pages 9 and 16.)

[41] Comoretto, G., Bertotti, B., Iess, L. and Ambrosini, R., "Doppler Experiments With the Cassini Radio System", Nuovo Cimento, 15, 1193-1198 (1992). [DOI]. (Cited on page 18.)

[42] Davies, R. W., "Issues in Gravitational Wave Detection with Space Missions", in Gravitational Waves and Radiations, Proceedings of the international conference, Université de Paris VII, Paris, France, June 18-22, 1973, Colloques Internationaux du CNRS, 220, pp. 33-45, (CNRS, Paris, France, 1974). (Cited on page 7.)

[43] Dixon, R. S. and Klein, C. A., "On the Detection of Unknown Signals", in Shostak, G. S., ed., Third Decennial US-USSR Conference on SETI, University of California, Santa Cruz, August 5-9, 1991, ASP Conference Series, 47, pp. 129-140, (Astronomical Society of the Pacific, San Francisco, CA, 1993). (Cited on page 34.)

[44] Dobrowolny, M. and Iess, L., "Interplanetary Plasma Turbulence and the Doppler Detection of a Gravitational Wave Background", Astron. Astrophys., 157, 346-352 (1986). [ADS]. (Cited on page 42.) 
[45] Edlund, J. A., Tinto, M., Królak, A. and Nelemans, G., "White-dwarf-white-dwarf galactic background in the LISA data", Phys. Rev. D, 71, 122003 (2005). [DOI], [ADS], [arXiv:gr-qc/0504112]. (Cited on page 48.)

[46] Estabrook, F. B., "Gravitational Wave Detection with the Solar Probe: II. The Doppler Tracking Method", in Neugebauer, M. and Davies, R. W., eds., A Close-Up of the Sun, Proceedings of the Solar Probe Science Workshop, Pasadena, California, 22-23 May 1978, JPL Publications, 78-70, pp. 441-449, (Jet Propulsion Laboratory, Pasadena, CA, 1978). (Cited on pages 8, 15, 17, 18, and 26.)

[47] Estabrook, F. B., "Response functions of free mass gravitational wave antennas", Gen. Relativ. Gravit., 17, 719-724 (1985). [DOI], [ADS]. (Cited on pages 8 and 11.)

[48] Estabrook, F. B. and Armstrong, J. W., "Three-Way Doppler Data for Gravitational Wave Searches", unpublished, (1983). Jet Propulsion Laboratory Interoffice Memorandum, 4/4/83. (Cited on page 44.)

[49] Estabrook, F. B., Armstrong, J. W., Tinto, M. and Folkner, W. M., "SyZyGy: A Straight Interferometric Spacecraft System for Gravity Wave Observations", Phys. Rev. D, 68, 062001 (2003). [DOI], [arXiv:gr-qc/0306071]. (Cited on page 11.)

[50] Estabrook, F. B., Hellings, R. W., Wahlquist, H. D. and Wolff, R. S., "Gravitational Radiation Detection with Spacecraft Doppler Tracking: Limiting Sensitivities and Prospective Missions", in Smarr, L. L., ed., Sources of Gravitational Radiation, Proceedings of the Battelle Seattle Workshop, July 24-August 4, 1978, pp. 37-48, (Cambridge University Press, Cambridge; New York, 1979). (Cited on page 8.)

[51] Estabrook, F. B., Tinto, M. and Armstrong, J. W., "Time-delay analysis of LISA gravitational wave data: Elimination of spacecraft motion effects", Phys. Rev. D, 62, 042002 (2000). [DOI], [ADS]. (Cited on pages 10 and 48.)

[52] Estabrook, F. B. and Wahlquist, H. D., "Response of Doppler spacecraft tracking to gravitational radiation", Gen. Relativ. Gravit., 6, 439-447 (1975). [DOI], [ADS]. (Cited on pages 7, 8, 10, 12, 15, 26, 30, 31, 34, 39, 41, and 42.)

[53] Freitag, M., "Gravitational Waves from Stars Orbiting the Sagittarius A* Black Hole", Astrophys. J. Lett., 583, L21-L24 (2003). [DOI], [ADS], [arXiv:astro-ph/0211209]. (Cited on page 40.)

[54] Giampieri, G. and Vecchio, A., "Limits on the Gravitational-Wave Background from Spacecraft Doppler Experiments", Class. Quantum Grav., 27, 793-811 (1995). [ADS]. (Cited on pages 31, 41, and 42.)

[55] Groth, E. J., "Probability Distributions Related to Power Spectra", Astrophys. J. Suppl. Ser., 29, 285-302 (1975). [DOI], [ADS]. (Cited on page 29.)

[56] Hasselmann, K., Munk, W. and MacDonald, G., "Bispectra of Ocean Waves", in Rosenblatt, M., ed., Proceedings of the Symposium on Time Series Analysis, Brown University, June 11-14, 1962, pp. 125-139, (Wiley, New York, 1963). (Cited on page 29.)

[57] Hellings, R. W., "Testing relativistic theories of gravity with spacecraft-Doppler gravity-wave detection", Phys. Rev. D, 17, 3158-3163 (1978). [DOI], [ADS]. (Cited on page 10.)

[58] Hellings, R. W., "Detecting a Gravitational-Radiation Background Using Spacecraft Doppler Tracking", Phys. Rev. Lett., 43, 470-473 (1979). [DOI], [ADS]. (Cited on page 42.)

[59] Hellings, R. W., personal communication, (1983). (Cited on page 44.)

[60] Hellings, R. W., Callahan, P. S., Anderson, J. D. and Moffet, A. T., "Spacecraft-Doppler gravity-wave detection. II. Results", Phys. Rev. D, 23, 844-851 (1981). [ADS]. (Cited on pages 37 and 41.) 
[61] Helstrom, C. W., Statistical Theory of Signal Detection, International Series of Monographs in Electronics and Instrumentation, 9, (Pergamon Press, Oxford; New York, 1968), 2nd edition. (Cited on pages 29, 30, and 34.)

[62] Hinkley, N. et al., "An Atomic Clock with 10 ${ }^{-18}$ Instability", Science, 341, 1215-1218 (2013). [DOI], [ADS], [1305.5869 [physics.atom-ph]]. (Cited on page 44.)

[63] Iess, L. and Armstrong, J. W., "Spacecraft Doppler Experiments", in Ciufolini, I. and Fidecaro, F., eds., Gravitational Waves: Sources and Detectors, Proceedings of the international conference, Cascina, Italy, 19-23 March, 1996, pp. 323-329, (World Scientific, Singapore; River Edge, NJ, 1997). (Cited on pages 29, 30, 34, 37, 41, and 42.)

[64] Iess, L., Armstrong, J. W., Bertotti, B., Wahlquist, H. D. and Estabrook, F. B., "Search for Gravitational Wave Bursts by Simultaneous Doppler Tracking of Three Interplanetary Spacecraft", Paper presented at 15th International Conference on General Relativity and Gravitation, Pune, India, December 16-21 1997, conference paper, (1997). (Cited on pages 37, 41, and 42.)

[65] Iess, L., Bonifazi, P., Bertotti, B. and Comoretto, G., "Differential Doppler Tracking of Interplanetary Spacecraft", Nuovo Cimento C, 10, 235-246 (1987). [DOI]. (Cited on pages 18, 20, and 24.)

[66] Iess, L. and Boscagli, G., "Advanced Radio Science Instrumentation for the Mission BepiColombo to Mercury", Planet. Space Sci., 49, 1597-1608 (2001). [DOI], [ADS]. (Cited on page 37.)

[67] Iess, L., di Benedetto, M., James, N., Mercolino, M., Simone, L. and Tortora, P., "Astra: Interdisciplinary Study on Enhancement of the End-to-End Accuracy for Spacecraft Tracking Techniques", Acta Astron., 94, 699-707 (2014). [DOI], [ADS]. (Cited on page 25.)

[68] Iess, L., Dobrowolny, M. and Bertotti, B., "Plasma effects on Doppler measurements of interplanetary spacecraft. I. Discontinuities and waves", Astron. Astrophys., 121, 203-210 (1983). [ADS]. (Cited on page 24.)

[69] Iess, L., Giampieri, G., Anderson, J. D. and Bertotti, B., "Doppler measurement of the solar gravitational deflection", Class. Quantum Grav., 16, 1487-1502 (1999). [DOI]. (Cited on pages 24 and 38.)

[70] Iess, L. et al., "The Cassini solar conjunction experiment: a new test of general relativity", in Proceedings of the IEEE Aerospace Conference 2003, Big Sky, MT, March 8-15, 2003, IEEE Conference Proceedings, pp. 1-211-223, (Institute of Electrical and Electronics Engineers, Piscataway, 2003). (Cited on pages 24 and 38.)

[71] Ishimaru, A., Wave Propagation and Scattering in Random Media, (Academic Press, New York, 1978). (Cited on pages 9, 16, and 18.)

[72] Jaffe, A. H. and Backer, D. C., "Gravitational Waves Probe the Coalescence Rate of Massive Black Hole Binaries", Astrophys. J., 583, 616-631 (2003). [DOI]. (Cited on pages 31 and 43.)

[73] Jenkins, G. M. and Watts, D. G., Spectral Analysis and Its Applications, (Holden-Day, San Francisco, 1969). (Cited on pages 9, 25, and 29.)

[74] Kaufmann, W. J., "Redshift Fluctuations Arising from Gravitational Waves", Nature, 327, 157-159 (1970). [DOI]. (Cited on page 7.)

[75] Keihm, S. J., Water Vapor Radiometer Measurements of the Tropospheric Delay Fluctuations at Goldstone Over a Full Year, TDA Progress Report, 42-122, (JPL, Pasadena, CA, 1995). (Cited on pages 20, 21, and 28.)

[76] Keihm, S. J., Tanner, A. and Rosenberger, H., "Measurements and Calibration of Tropospheric Delay at Goldstone From the Cassini Media Calibration System", IPN Progress Report, 2004, 42-158 (2004). URL (accessed 5 August 2005):

http://tmo.jpl.nasa.gov/progress_report/42-158/158A.pdf. (Cited on pages 18, 20, 21, and 38.) 
[77] Kellerman, K. and Sheets, B., eds., Serendipitous Discoveries in Radio Astronomy, Proceedings of a Workshop held at NRAO, Greenbank, USA, May 4-6, 1983, (National Radio Astronomy Observatory, Green Bank, 1983). (Cited on page 36.)

[78] Kliore, A. et al., "Cassini Radio Science", Space Sci. Rev., 115, 1-70 (2004). [DOI], [ADS]. (Cited on pages 14,24 , and 37.$)$

[79] Lovelace, R. V.E., Theory and Analysis of Interplanetary Scintillations, Ph.D. thesis, (Cornell University, Ithaca, NY, 1970). (Cited on pages 9 and 16.)

[80] MacDonald, G. J., "Spectral Analysis of Time Series Generated by Nonlinear Processes", Rev. Geophys., 27, 449-469 (1989). [DOI]. (Cited on page 29.)

[81] MacMillan, D. S. and Gipson, J. M., "Atmospheric Pressure Loading Parameters from Very Long Baseline Interferometry Observations", J. Geophys. Res., 99(B9), 18081-18088 (1994). [DOI], [ADS]. (Cited on page 22.)

[82] Mashhoon, B. and Grishchuk, L. P., "On the Detection of a Stochastic Background of Gravitational Radiation by the Doppler Tracking of Spacecraft", Astrophys. J., 236, 990-999 (1980). [DOI], [ADS]. (Cited on page 42.)

[83] Matousek, S., "The Juno New Frontiers Mission", Acta Astron., 61, 932-939 (2007). [DOI], [ADS]. (Cited on page 37.)

[84] Misner, C. W., Thorne, K. S. and Wheeler, J. A., Gravitation, (W.H. Freeman, San Francisco, 1973). [ADS]. (Cited on page 10.)

[85] Moyer, T. D., Mathematical Formulation of the Double-Precision Orbit Determination Program (DPODP), JPL Technical Report, 32-1527, (JPL / NASA, Pasadena, CA, 1971). Online version (accessed 2 January 2016): http://hdl.handle.net/2060/19710017134. (Cited on page 25.)

[86] Otoshi, T. Y. and Franco, M. M., "Measurements of a Deep Space Station Fractional Frequency Stability to the $10^{-15}$ Level", IEEE Trans. Instrum. Meas., 41, 577-587 (1992). [DOI]. (Cited on page 22.)

[87] Otoshi, T. Y., Franco, M. N. and Lutes, G. F., "The Fractional Frequency Stability of a 34-m Diameter Beam Waveguide Antenna", Proc. IEEE, 82, 788-795 (1994). [DOI]. (Cited on page 22.)

[88] Papoulis, A., Probability, Random Variables, and Stochastic Processes, (McGraw-Hill, New York, 1965). (Cited on pages 9 and 29.)

[89] Percival, D. B. and Walden, A. T., Spectral Analysis for Physical Applications, (Cambridge University Press, Cambridge, 1993). [Google Books]. (Cited on pages 9, 25, and 29.)

[90] Pitkin, M., Reid, S., Rowan, S. and Hough, J., "Gravitational Wave Detection by Interferometry (Ground and Space)", Living Rev. Relativity, 14, lrr-2011-5 (2011). [DOI], [ADS]. URL (accessed 2 January 2016): http://www.livingreviews.org//rr-2011-5. (Cited on page 47.)

[91] Press, W. H. and Thorne, K. S., "Gravitational-Wave Astronomy", Annu. Rev. Astron. Astrophys., 10, 335-373 (1972). [DOI], [ADS]. (Cited on page 7.)

[92] Prince, T. A., Tinto, M., Larson, S. L. and Armstrong, J. W., "LISA optimal sensitivity", Phys. Rev. D, 66, 122002 (2002). [DOI], [ADS], [arXiv:gr-qc/0209039]. (Cited on page 48.)

[93] Rajagopal, M. and Romani, R. W., "Ultra-Low-Frequency Gravitational Radiation from Massive Black Hole Binaries", Astrophys. J., 446, 543-549 (1995). [DOI]. (Cited on pages 31 and 43.) 
[94] Resch, G. M., Bar-Server, Y., Keihm, S. J., Kroger, P., Linfield, R. P., Mahoney, M. J., Tanner, A. and Teitelbaum, L. P., "Atmospheric calibration for precision Doppler tracking of spacecraft", Planet. Space Sci., 46, 1257-1261 (1998). [DOI], [ADS]. (Cited on pages 18, 21, and 38.)

[95] Resch, G. M., Hogg, D. E. and Napier, P. J., "Radiometric Correction of Atmospheric Path-Length Fluctuations in Interferometric Experiments", Radio Sci., 19, 411-422 (1984). [DOI]. (Cited on pages 18 and 21.)

[96] Resch, G. M., Keihm, S. J., Lanyi, G. E., Linfield, R. P., Naudet, C. J., Riley, A. L., Rosenberger, H. W. and Tanner, A. B., "The Media Calibration System for Cassini Radio Science: Part III", IPN Progress Report, 2002, 42-148 (2002). URL (accessed 5 August 2005):

http://tmo.jpl.nasa.gov/progress_report/42-148/148H.pdf. (Cited on pages 18, 21, 28, and 38.)

[97] Rice, S. O., "Mathematical Analysis of Random Noise", in Wax, N., ed., Selected Papers on Noise and Stochastic Processes, pp. 133-294, (Dover, New York, 1954). (Cited on pages 29 and 39.)

[98] Richie-Halford, A. C., Iess, L., Tortora, P., Armstrong, J. W., Asmar, S. W., Woo, R., Habbal, S. R. and Morgan, H., "Space-Time Localization of Inner Heliospheric Plasma Turbulence Using Multiple Spacecraft Links", Space Weather, 7, S12003 (2009). [DOI], [ADS]. (Cited on page 17.)

[99] Rickett, B. J., "Interstellar Scattering and Scintillation of Radio Waves", Annu. Rev. Astron. Astrophys., 15, 479-504 (1977). [DOI], [ADS]. (Cited on pages 9 and 16.)

[100] Riley, A. L. et al., Cassini Ka-Band Precision Doppler and Enhanced Telecommunications System Study, (NASA/JPL and Agenzia Spaziale Italiana, Pasadena, CA; Rome, 1990). (Cited on pages 22 and 25.)

[101] Shaddock, D. A., "Operating LISA as a Sagnac interferometer", Phys. Rev. D, 69, 022001 (2004). [DOI], [ADS]. (Cited on page 47.)

[102] Smith, S., "Algorithm to search for gravitational radiation from coalescing binaries", Phys. Rev. D, 36, 2901-2904 (1987). [DOI], [ADS]. (Cited on page 30.)

[103] Snel, R. C., Mangum, J. G. and Baars, J. W.M., "Study of the Dynamics of Large Reflector Antennas With Accelerometers", IEEE Trans. Ant. Prop., 49, 84-101 (2007). [DOI], [arXiv:0710.4254]. (Cited on page 46.)

[104] Sovers, O. J., Fanselow, J. L. and Jacobs, C. S., "Astrometry and geodesy with radio interferometry: experiments, models, results", Rev. Mod. Phys., 70, 1393-1454 (1998). [DOI], [ADS], [astroph/9712238]. (Cited on page 22.)

[105] Study of the Laser Interferometer Space Antenna - Final Technical Report, (NASA, Pasadena, CA, 2000). Online version (accessed 11 January 2016):

http://lisa.nasa.gov/documentation.html. (Cited on page 47.)

[106] Tatarski, V. I., Wave Propagation in a Turbulent Medium, (Dover, New York, 1961). (Cited on pages 9 and 16.)

[107] Thomson, D. J., "Spectrum Estimation and Harmonic Analysis", Proc. IEEE, 70, 1055-1096 (1982). [DOI]. (Cited on page 29.)

[108] Thorne, K. S., "Gravitational radiation", in Hawking, S. W. and Israel, W., eds., Three Hundred Years of Gravitation, pp. 330-458, (Cambridge University Press, Cambridge; New York, 1987). [Google Books]. (Cited on pages 7, 31, 36, and 41.)

[109] Thorne, K. S. and Braginsky, V. B., "Gravitational-wave bursts from nuclei of distant galaxies and quasars: Proposal for detection using doppler tracking of interplanetary spacecraft", Astrophys. J., 204, L1-L6 (1976). [DOI], [ADS]. (Cited on page 8.) 
[110] Tinto, M., "Spacecraft Doppler tracking as a xylophone detector of gravitational radiation", Phys. Rev. D, 53, 5354-5364 (1996). [DOI], [ADS]. (Cited on page 44.)

[111] Tinto, M., "The Cassini Ka-band gravitational wave experiments", Class. Quantum Grav., 19, 17671773 (2002). [DOI]. (Cited on pages 27, 28, and 40.)

[112] Tinto, M. and Armstrong, J. W., "Coincidence Probabilities for Spacecraft Gravitational Wave Experiments: Massive Coalescing Binaries", Astrophys. J., 372, 545-553 (1991). [DOI], [ADS]. (Cited on pages 30 and 41.)

[113] Tinto, M. and Armstrong, J. W., "Spacecraft Doppler tracking as a narrow-band detector of gravitational radiation", Phys. Rev. D, 58, 042002 (1998). [ADS]. (Cited on page 38.)

[114] Tinto, M., Armstrong, J. W. and Estabrook, F. B., "Discriminating a gravitational wave background from instrumental noise in the LISA detector", Phys. Rev. D, 63, 021101(R) (2001). [DOI], [ADS]. (Cited on page 48.)

[115] Tinto, M., DeBra, D., Buckman, S. and Tilley, S., "gLISA: geosynchronous laser interferometer space antenna concepts with off-the-shelf satellites", Rev. Sci. Instrum., 86, 014501 (2015). [DOI], [ADS], [arXiv:1410.1813 [gr-qc]]. (Cited on page 47.)

[116] Tinto, M. and Dhurandhar, S. V., "Time-Delay Interferometry", Living Rev. Relativity, 17, lrr-20146 (2014). [DOI], [ADS]. URL (accessed 13 October 2014): http://www.livingreviews.org/lrr-2014-6. (Cited on page 48.)

[117] Tinto, M., Dick, G. J., Prestage, J. D. and Armstrong, J. W., "Improved spacecraft radio science using and on-board atomic clock: Application to gravitational wave searches", Phys. Rev. D, 79, 102003 (2009). [DOI], [ADS], [arXiv:0812.2581 [gr-qc]]. (Cited on page 44.)

[118] Tinto, M., Estabrook, F. B. and Armstrong, J. W., Time-Delay Interferometry and LISA's Sensitivity to Sinusoidal Gravitational Waves, (JPL / Caltech, Pasadena, CA, 2002). Online version (accessed 11 January 2016):

http://list.caltech.edu/doku.php?id=mission_documents. (Cited on page 48.)

[119] Tinto, M., Estabrook, F. B. and Armstrong, J. W., "Time-delay interferometry for LISA", Phys. Rev. D, 65, 082003 (2002). [DOI], [ADS]. (Cited on page 10.)

[120] Tinto, M., Estabrook, F. B. and Armstrong, J. W., "Time-delay interferometry with moving spacecraft arrays", Phys. Rev. D, 69, 082001 (2004). [DOI], [arXiv:gr-qc/0310017]. (Cited on page 10.)

[121] Tortora, P., Iess, L., Bordi, J. J., Ekelund, J. E. and Roth, D. C., "Precise Cassini Navigation During Solar Conjunctions Through Multifrequency Plasma Calibrations", J. Guid. Control Dyn., 27, 251-257 (2004). [DOI]. (Cited on pages 18, 20, 21, and 38.)

[122] Tortora, P., Iess, L. and Ekelund, J. E., "Accurate Navigation of Deep Space Probes using Multifrequency Links: the Cassini Breakthrough During Solar Conjunction Experiments", IAF abstracts, 34th COSPAR Scientific Assembly, The Second World Space Congress, held 10-19 October, 2002 in Houston, TX, USA, p.Q-2-03, conference paper. [ADS]. (Cited on pages 18, 20, 21, and 38.)

[123] van Dam, T. M., Blewitt, G. and Heflin, M. B., "Atmospheric Pressure Loading Effects on Global Positioning System Coordinate Determinations", J. Geophys. Res., 99(B12), 23939-23950 (1994). [DOI], [ADS]. (Cited on page 22.)

[124] Vecchio, A., Bertotti, B. and Iess, L., "Coalescing Binaries and Doppler Experiments", in Coccia, E., Veneziano, G. and Pizzella, G., eds., Second Edoardo Amaldi Conference on Gravitational Waves, Proceedings of the conference held at CERN, Switzerland, 1-4 July, 1997, Edoardo Amaldi Foundation Series, 4, p. 272, (World Scientific, Singapore; River Edge, NJ, 1998). [ADS], [arXiv:grqc/9708033]. (Cited on page 42.) 
[125] Vessot, R. F.C. and Levine, M. W., "A Time-Correlated Four-Link Doppler Tracking System", in Neugebauer, M. and Davies, R. W., eds., A Close-Up of the Sun, Proceedings of the Solar Probe Science Workshop, Pasadena, California, 22-23 May 1978, JPL Publications, 78-70, pp. 457-497, (Jet Propulsion Laboratory, Pasadena, CA, 1978). (Cited on pages 15, 17, and 44.)

[126] Vessot, R. F.C. et al., "Test of Relativistic Gravitation with a Space-Borne Hydrogen Maser", Phys. Rev. Lett., 45, 2081-2084 (1980). [DOI], [ADS]. (Cited on page 7.)

[127] Wahlquist, H. D., "The Doppler response to gravitational waves from a binary star source", Gen. Relativ. Gravit., 19, 1101-1113 (1987). [DOI], [ADS]. (Cited on pages 30, 34, 39, and 40.)

[128] Wahlquist, H. D., Anderson, J. D., Estabrook, F. B. and Thorne, K. S., "Recent JPL Work on Gravity Wave Detection and Solar System Relativity Experiments", in International Meeting on Experimental Gravitation, Simposio internazionale sulla gravitazione sperimentale, Pavia, Italy, 17 20 September 1976, Atti dei Convegni Lincei, 34, pp. 335-350, (Accad. Naz. dei Lincei, Rome, 1977). (Cited on pages 8 and 18.)

[129] Won, L., Hanover, G., Belenky, R. and Lee, A., "Supporting the Gravity Wave Experiment: Three AACS Topics", unpublished, (2001). Jet Propulsion Laboratory, Cassini Project presentation October 18, 2001. (Cited on pages 25, 26, and 28.)

[130] Won, L. and Lee, A., "AACS Support for the Gravity Wave Experiment", unpublished, (2001). Jet Propulsion Laboratory Interoffice Memorandum SCO-01-044, 10/29/01. (Cited on page 28.)

[131] Woo, R. and Armstrong, J. W., "Spacecraft Radio Scattering Observations of the Power Spectrum of Electron Density Fluctuations in the Solar Wind", J. Geophys. Res., 84, 7288-7296 (1979). [DOI]. (Cited on pages 14, 17, and 20.)

[132] Wyithe, J. S.B. and Loeb, A., "Low-Frequency Gravitational Waves from Massive Black Hole Binaries: Predictions for LISA and Pulsar Timing Arrays", Astrophys. J., 590, 691-706 (2003). [DOI], [ADS]. (Cited on page 43.)

[133] Zannoni, M. and Tortora, P., "Numerical Error in Interplanetary Orbit Determination Software", $J$. Guid. Control Dyn., 36, 1008-1018 (2013). [DOI], [ADS]. (Cited on page 25.) 